\title{
ENGINEERING SACCHAROMYCES FOR BIOFUEL PRODUCTION IMPROVEMENT
}

\section{QIU ZILONG}

School of Chemical and Biomedical Engineering

A thesis submitted to Nanyang Technological University in fulfillment of the requirement for the degree of Doctor of Philosophy 


\section{Acknowledgements}

Here, I want to thank all the people that have helped me in the preparation of this thesis.

First of all, I would like to thank my previous and current supervisor, assistant professor Jiang Rongrong and associate professor Raymond Lau Wai Man. Professor Jiang Rongrong has directed most of my projects and I have learned so much from professor Jiang throughout my PhD study, including research design, paper editing, working attitude and etc. Professor Raymond has helped me a lot in the last period of my $\mathrm{PhD}$ program and has always been supportive for the preparation of my thesis. Without their valuable enlightenment and guidance, I could not be able to write this thesis.

Secondly, I would like to thank my seniors, Dr. Hefang Geng and Dr. Liu Wenshan, who taught me lots of techniques and skills in bioengineering. Also, many thanks to other colleagues in our group, including Dr. Cao Xitao, Dr. Zhang Bo, and Ms. Zhong Yang. They offered kind help when I encountered experimental difficulties and also created pleasant environment in our lab.

Last but not least, I would like to give my gratitude to my family and my friends. Thanks to their continuous encouragement and support, I could go through the hard time and be faced to the future optimistically. They always give me useful suggestions and comments to encourage me to become a sincere, diligent and helpful person. I cannot finish my whole study without them. 


\section{Table of contents}

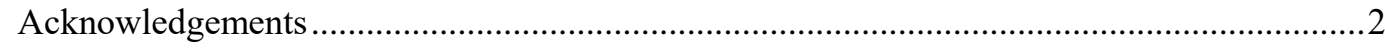

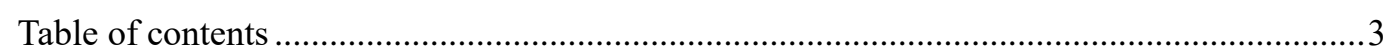

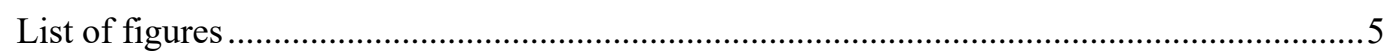

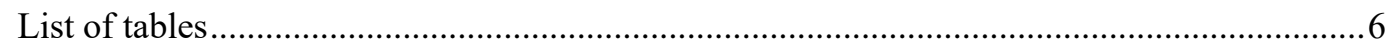

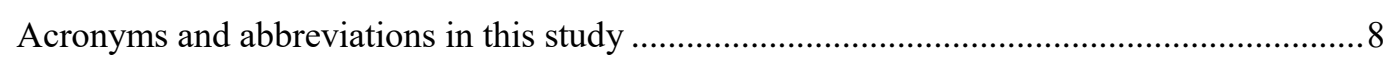

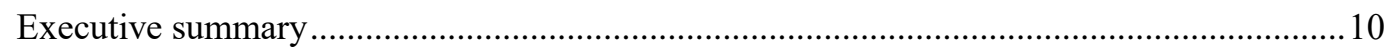

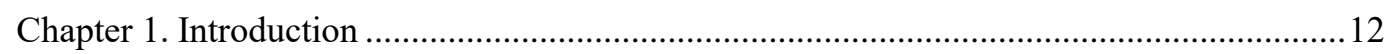

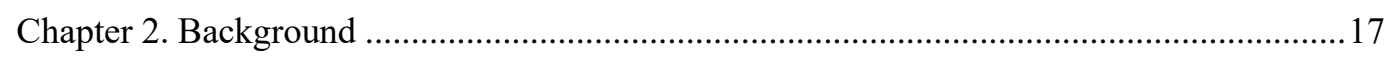

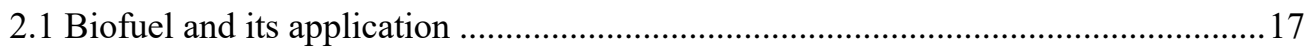

2.2 Saccharomyces cerevisae as host strain in biofuel production...................................18

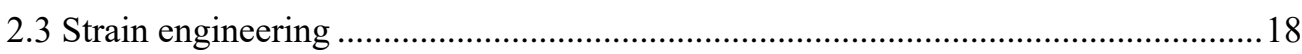

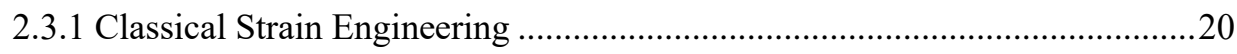

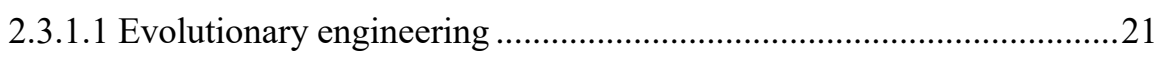

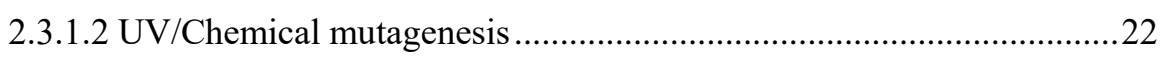

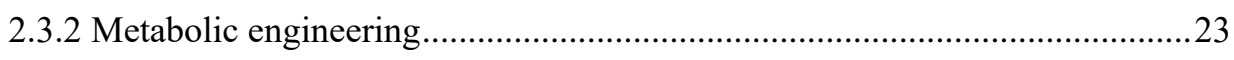

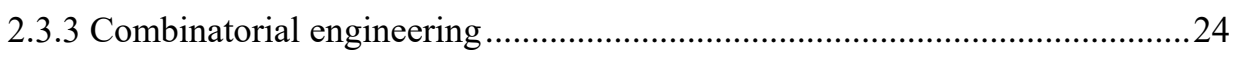

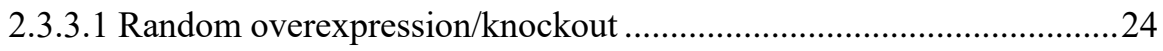

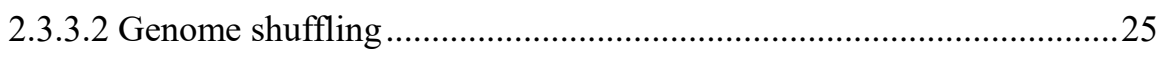

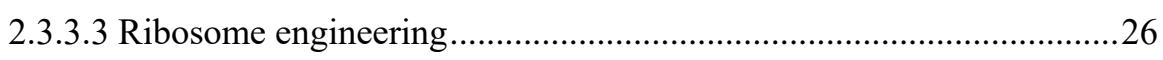

2.3.3.4 Global transcription machinery engineering (gTME) .........................2

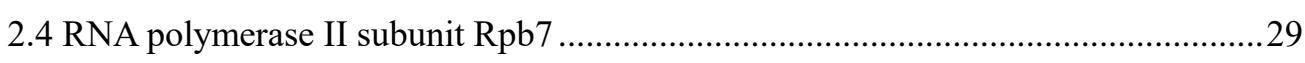

2.5 Metabolic engineering approaches to increase fatty acid production..........................31

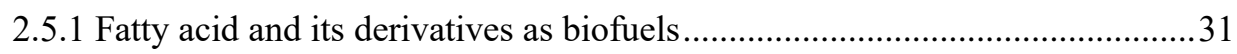

2.5.2 Fatty acids synthesis in Saccharomyces cerevisae .............................................

2.5.3 Fatty acids production through overproducing acetyl-CoA .............................32

2.5.3.1 Redirect the glycolytic flux from ethanol synthesis to acetyl-CoA

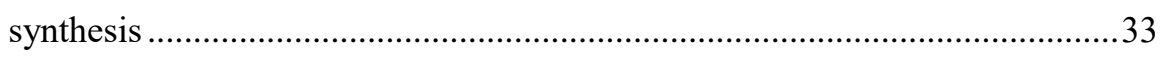

2.5.3.2 Inactivate acetyl-CoA consuming pathways .......................................... 33

2.5.3.3 Engineering PDH-bypass pathway.........................................................

2.5.3.4 ATP-dependent citrate lyase (ACL) ......................................................... 34

2.5.3.5 Pyruvate dehydrogenase (PDH) pathway.................................................

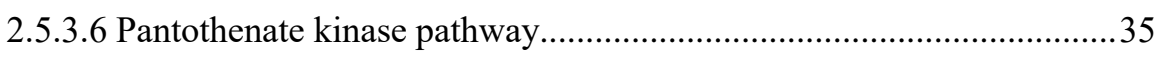

2.5.4 Fatty acids production through engineering the step from acetyl-CoA to

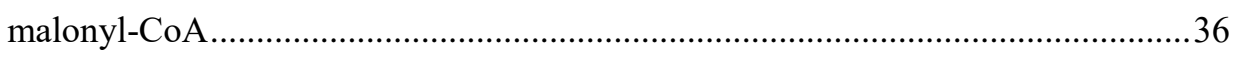

2.5.5 Fatty acids production through engineering malonyl-CoA synthetase pathway

Chapter 3. Improving Saccharomyces cerevisiae ethanol production and tolerance via RNA

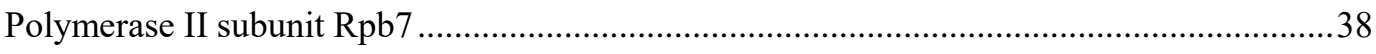

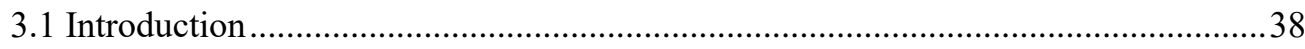

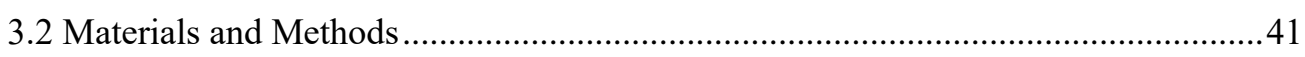

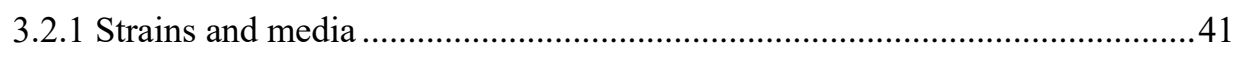

3.2.2 Plasmid and mutant library construction....................................................... 


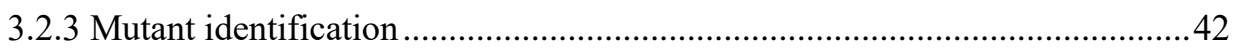

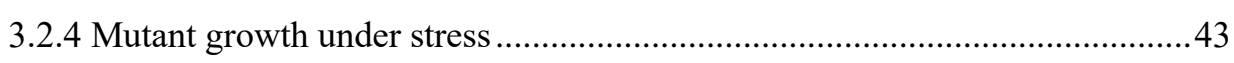

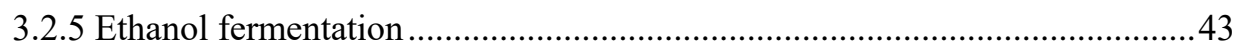

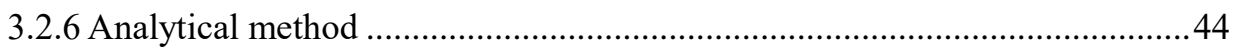

3.2.7 DNA Microarray and quantitative Real-Time Reverse Transcription PCR (qRT-

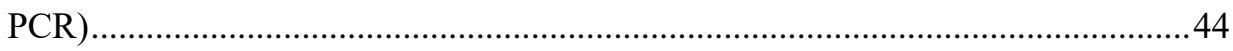

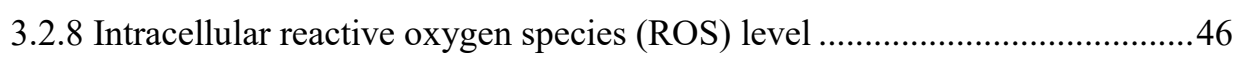

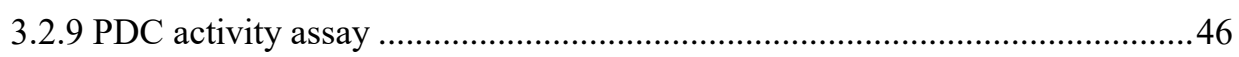

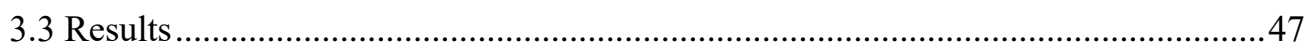

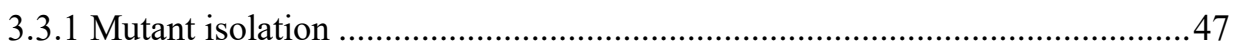

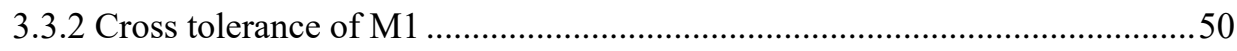

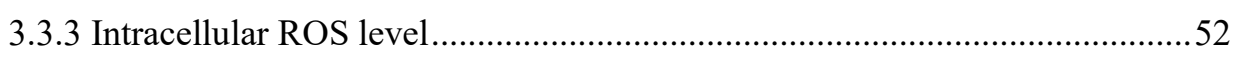

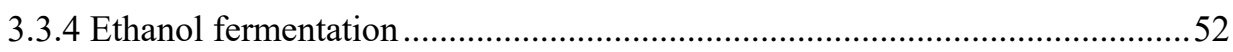

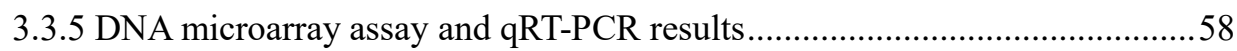

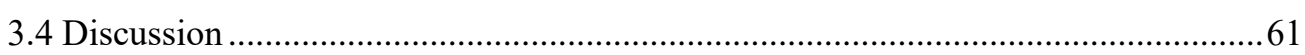

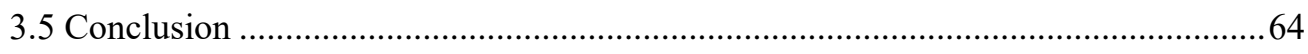

Chapter 4. Systematic engineering for improving Saccharomyces cerevisiae alcohol tolerance

4.1 Introduction.

4.2 Bioalcohol toxicity/tolerance mechanism of $S$. cerevisiae and their respective

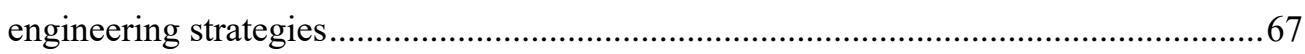

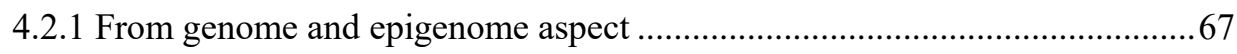

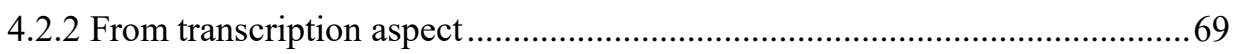

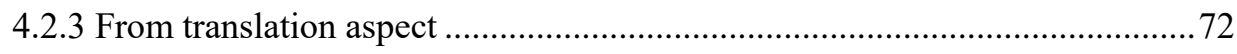

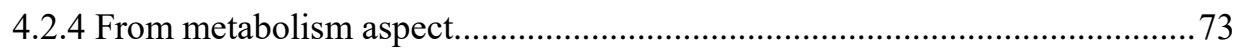

4.2.5 From plasma membrane aspect................................................................... 75

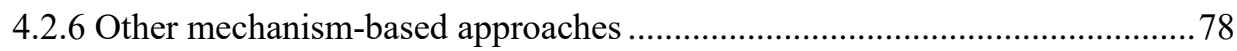

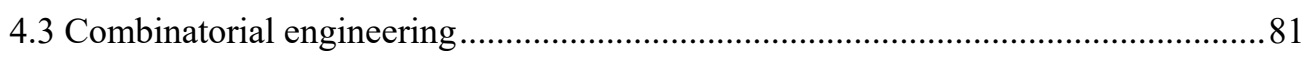

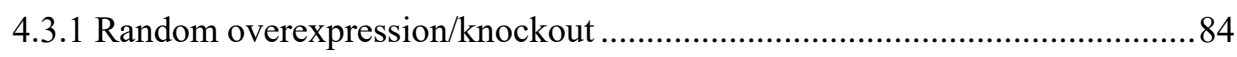

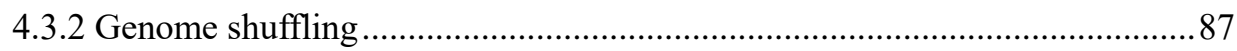

4.3.3 Global transcription machinery engineering (gTME) ...................................... 88

4.3.4 Adaptive evolution \& hybridization ............................................................

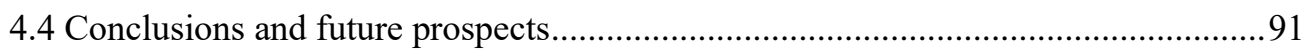

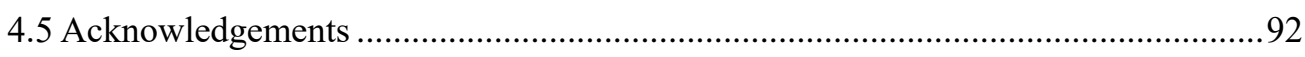

Chapter 5. Improving fatty acids production through enhancing the precursor malonyl-CoA

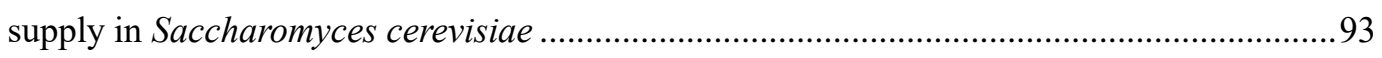

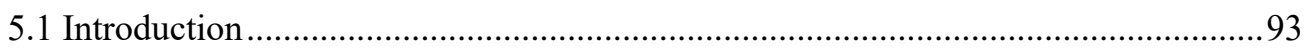

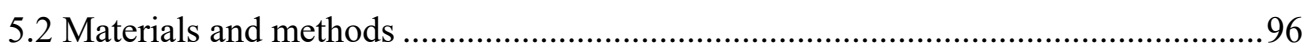

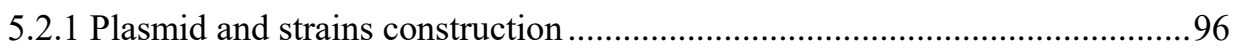

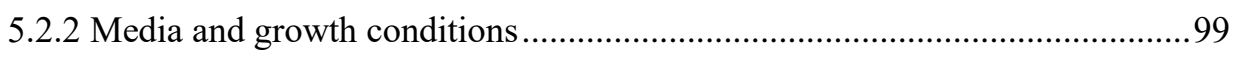

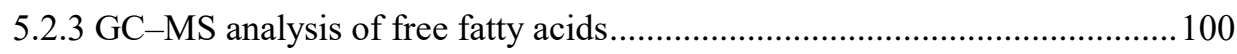

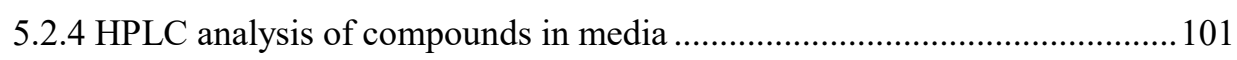

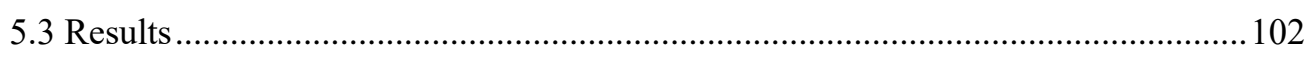

5.3.1 Growth rates of the wild-type strain and engineered strains ...........................102 
5.3.2 Fatty acids production in strains with enhanced acetyl-CoA carboxylase pathway

5.3.3 Fatty acids production in strains with enhanced malonyl-CoA synthetase pathway 104

5.3.4 Fatty acids production without malonic acids supply 106

5.3.5 The utilization of malonic acids in engineered strains 107

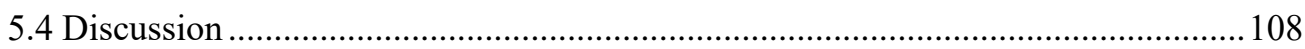

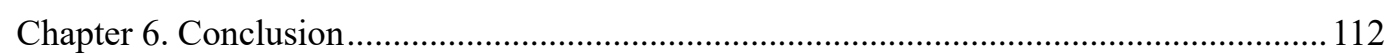

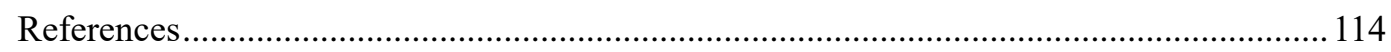

\section{List of figures}

Figure 1. Schematic diagram showing the general workflow of traditional strain engineering.....21

Figure 2. The workflow of gTME. .28

Figure 3. Models of Rpb7 multifunction in gene expression........................ 30

Figure 4. Design and construction of acetyl-CoA overproducing yeast strains ...............33

Figure 5. Ethanol tolerance.

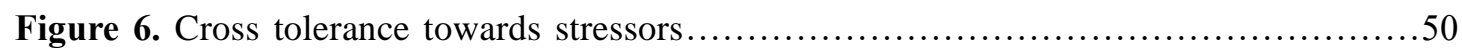

Figure 7. Cross tolerance towards inhibitors from lignocellulose hydrolysates.............51

Figure 8. Intracellular ROS level in M1when cells reached early log phase...................52

Figure 9. Fermentation characteristics during VHG laboratory fermentation..................53

Figure 10. Ethanol profile with varying initial glucose supply under VHG fermentation.........55

Figure 11. Ethanol profile with varying initial $\mathrm{pH}$ under $\mathrm{VHG}$ fermentation. ..................56

Figure 12. Fermentation characteristics in yeast strains...............................57

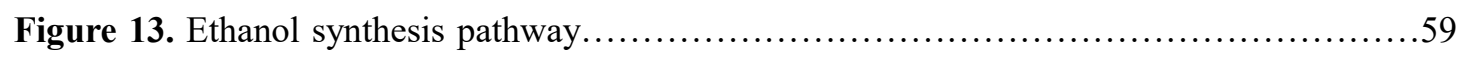

Figure 14. PDC activity in M1 and the control after $12 \mathrm{~h}$ VHG fermentation....................63

Figure 15. Strategic workflow of Saccharomyces cerevisiae engineering to improve its bioalcohol

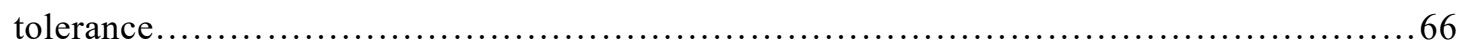


Figure 16. Diagram of the combinatorial engineering approaches implemented in S. cerevisiae to improve alcohol tolerance

Figure 17. Simplified overview of the engineering strategy to enhance the acetyl-CoA carboxylase pathway (overexpression of PanK, $A L D 6, \operatorname{Se} A c s^{L 641 P}$ and $A C C 1^{S 659 A, S 1157 A}$ ) and malonyl-CoA synthetase pathway (introduction of $M A E 1$ and $M a t B$ in $S$. cerevisiae) to increase the supply of malonyl-CoA precursors for fatty acids production. .96

Figure 18. Growth profile of engineered strains in CM medium with malonic acids supply .102

Figure 19. Fatty acids production of strains CEN-K-6, CEN-PAA-K, CEN-PAA-C, CEN-PAA$\mathrm{AB}$ and $\mathrm{CEN}-\mathrm{PAA}-\mathrm{ABC}$ in CM medium with malonic acids supply after $24 \mathrm{~h}$ 104

Figure 20. Fatty acids production of strains CEN-K-6, CEN-A, CEN-B, CEN-AB, CEN-PAA-AB and CEN-PAA-ABC in CM medium with malonic acids supply after $24 \mathrm{~h}$. Error bars represent the standard errors of three replicates. .105

Figure 21. Fatty acids production of engineered strains CEN-K-6, CEN-PAA-K, CEN-PAA-C, CEN-PAA-AB and CEN-PAA-ABC in CM medium without malonic acids supply after 24h...106

Figure 22. Malonic acids concentration in the medium of CEN-K-6, CEN-PAA-K, CEN-PAA-C, CEN-PAA-AB and CEN-PAA-ABC during the culturing process. .106

\section{List of tables}

Table 1. Primers used in plasmid construction and error-prone PCR.

Table 2. Primers used in qRT-PCR. .45

Table 3. Fermentation profiles of M1 and the control after $54 \mathrm{~h}$. .54

Table 4. Differentially expressed genes in M1 after $12 \mathrm{~h}$ VHG fermentation. .60

Table 5. Comparison between DNA microarray and qRT-PCR results on selected genes from M1 
after $12 \mathrm{~h}$ VHG fermentation

Table 6. Examples of metabolism-based engineering approaches to improve S. cerevisiae bioalcohol tolerance. .73

Table 7. Examples of plasma membrane-based engineering approaches to improve S. cerevisiae bioalcohol tolerance. .76

Table 8. Examples of other mechanism-based engineering approaches to improve S. cerevisiae bioalcohol tolerance. .79

Table 9. Examples of random overexpression/knockout approaches to improve S. cerevisiae bioalcohol tolerance. .84

Table 10. Plasmids in this study .98

Table 11. Primers used in this study. .98

Table 12. Strains in this study. .99 
Acronyms and abbreviations in this study.

\begin{tabular}{|c|c|c|}
\hline Abbreviation & Full name & Remarks \\
\hline S. cerevisiae & Saccharomyces cerevisiae & Host strain in this study \\
\hline RNAP II & RNA polymerase II & - \\
\hline VHG & very high gravity & $\begin{array}{l}\text { An approach of ethanol fermentation in } \\
\text { industry }\end{array}$ \\
\hline gTME & $\begin{array}{l}\text { global transcription machinery } \\
\text { engineering }\end{array}$ & $\begin{array}{l}\text { A kind of combinatorial engineering } \\
\text { method }\end{array}$ \\
\hline TFs & transcription factors & - \\
\hline Pank & pantothenate kinase & - \\
\hline IEA & International Energy Agency & - \\
\hline FAEEs & fatty acid ethyl esters & $\begin{array}{l}\text { A type of ester that result from the } \\
\text { combination of a fatty acid with an alcohol }\end{array}$ \\
\hline IME & inverse metabolic engineering & - \\
\hline NTG & $\begin{array}{l}\text { N-methyl-N'-nitro-N- } \\
\text { nitrosoguanidine }\end{array}$ & A kind of chemical mutagen \\
\hline EMS & ethyl methanesulfonate & A kind of chemical mutagen \\
\hline GPM & genotype-phenotype map & $\begin{array}{l}\text { A study of the relationship between } \\
\text { genotype and phenotype }\end{array}$ \\
\hline CRISPR & $\begin{array}{l}\text { clustered regularly interspaced } \\
\text { short palindromic repeats }\end{array}$ & A family of DNA sequences \\
\hline HEPE & $\begin{array}{l}\text { high-energy pulse electron } \\
\text { beam }\end{array}$ & A method of introducing mutation \\
\hline FACS & $\begin{array}{l}\text { fluorescence-activated cell } \\
\text { sorting }\end{array}$ & A kind of cell sorting approach \\
\hline CTD & C-terminal domain & - \\
\hline FFA & free fatty acids & - \\
\hline FAL & fatty alcohols & - \\
\hline FAB & fatty acid biosynthesis & - \\
\hline FAS & fatty acids synthase & - \\
\hline $\mathrm{PDH}$ & pyruvate dehydrogenase & - \\
\hline $\mathrm{ADH}$ & alcohol dehydrogenase & - \\
\hline 3-HP & 3-hydroxypropionic acid & - \\
\hline ALD & aldehyde dehydrogenase & - \\
\hline $\mathrm{ACL}$ & ATP-citrate lyase & - \\
\hline $\mathrm{ACC}$ & Acetyol-CoA carboxylase & - \\
\hline CRP & cAMP receptor protein & A kind of transcription factor \\
\hline LB & lysogeny broth & A kind of culture medium \\
\hline HMF & hydroxymethylfurfural & - \\
\hline RID & refractive Index Detector & A kind of HPLC detector \\
\hline
\end{tabular}




\begin{tabular}{|l|l|l|}
\hline H2DCFDA & $\begin{array}{l}2^{\prime}, 7^{\prime}- \\
\text { dichlorodihydrofluoroscein } \\
\text { diacetate }\end{array}$ & - \\
\hline PPB & potassium phosphate buffer & - \\
\hline ROS & reactive oxygen species & $\begin{array}{l}\text { Reactive chemical species containing } \\
\text { oxygen }\end{array}$ \\
\hline DCW & dry cells weight & - \\
\hline RNP & ribonucleoprotein & - \\
\hline GO & gene ontology & - \\
\hline V-ATPase & vacuolar H ${ }^{+}$-ATPase & - \\
\hline QTL & quantitative trait loci & $\begin{array}{l}\text { A section of DNA that correlates with } \\
\text { variation in a phenotype }\end{array}$ \\
\hline HDAC & histone acetylation & - \\
\hline H3K4 & $\begin{array}{l}\text { lysine } 4 \text { methylation of histone } \\
\text { H3 }\end{array}$ & $\begin{array}{l}\text { A mechanism for modifying chromatin } \\
\text { structure }\end{array}$ \\
\hline HSP & heat shock protein & $\begin{array}{l}\text { A family of proteins in response to } \\
\text { exposure to stress }\end{array}$ \\
\hline STRE & stress response elements & - \\
\hline ABC & ATP-Binding Cassette & - \\
\hline
\end{tabular}




\section{Executive summary}

The continuous use of fossil fuels has led to many issues such as energy crisis, environmental change and etc. People are seeking cleaner and more sustainable energy resources to replace traditional fossil fuels. Microbial conversion of renewable feedstock into biofuels and chemicals has been investigated extensively in recent decades. Saccharomyces cerevisiae (S. cerevisiae) is one the most popular microbial factory applied for producing valuable chemical products. Its advantages in industrial application comes from its biological properties such as post-translational modifications, less possibility of contamination, robustness towards harsh industrial condition, good tolerance to inhibitory compounds and etc. Among various biofuels, bioethanol is a natural product of yeast. Moreover, fatty acids and their derivatives are energy-rich molecules and considered as excellent candidates for renewable liquid transport fuels and chemicals.

In this study, transcription engineering on RNA polymerase II (RNAP II) was conducted to improve S. cerevisiae ethanol tolerance and production. Error-prone PCR was applied to engineer subunit Rpb7 of RNAP II. Random mutagenesis library of Rpb7 was constructed and subjected to screening under ethanol stress. The isolated variant M1 showed much improved resistance towards $8 \%$ and $10 \%$ ethanol. The ethanol titers of M1 was $\sim 122 \mathrm{~g} / \mathrm{L}(96.58 \%$ of the theoretical yield) under laboratory very high gravity (VHG) fermentation, about $40 \%$ increase as compared to the control. DNA microarray assay showed that 369 genes had differential expression in M1 after $12 \mathrm{~h}$ VHG fermentation, which are involved in glycolysis, alcoholic fermentation, 
oxidative stress response and etc. The systematic engineering approaches for improving S. cerevisiae alcohol tolerance were discussed next. As for fatty acids production improvement, acetyl-CoA carboxylase pathway coupled with malonyl-CoA synthetase pathway were introduced into S. cerevisiae. After $24 \mathrm{~h}$ fermentation with the supply of $0.38 \%(\mathrm{w} / \mathrm{v})$ malonic acids, the engineered strains with enhancement in acetyl-CoA carboxylase pathway and malonyl-CoA synthetase pathway showed much improvement in fatty acids production. Specifically, the accumulated C16:0 and C18:0 in the engineered strain CEN-PAA-AB with two pathways combined reached 86.74 and $86.97 \mathrm{ug} / 10^{8}$ cells respectively, which was about five-fold of the wild strain.

The research in thesis has successfully improved two kinds of biofuels (ethanol and fatty acids) in $S$. cerevisiae through different engineering strategies. It firstly demonstrated that eukaryotic RNAP II enzyme could be an alternative gTME target in eliciting improved phenotypes. In addition, the combination of malonyl-CoA pathway with the acetyl-CoA pathway has been proved as a valid platform for improving advanced biofuel production. 


\section{Chapter 1. Introduction}

Microbes have long been used for the production of various non-natural compounds, such as medical drugs, food additives, enzyme, biofuels. In recent decades, some global issues such as limited supply of fossil fuels, constant environment deterioration and the climate change have stimulated much intention and initiative to increase the production of eco-friendly renewable sources. As renewable liquid transport fuels, biofuels have been intensively investigated to improve their industrial production.

Among various microbes applied for biofuels production, S. cerevisiae is one of the most popular microbial factories in the industry. It has been widely used as superb bioalcohol producer by utilizing various feedstocks and exploited as a potential candidate workhorse for many other biofuels compounds production. Among different biofuels produced by $S$. cerevisiae, ethanol is a natural product of yeast and it is compatible with the existing combustion engines. In addition, renewable synthesis of microbial fatty acids and their derivatives are quite promising since they are energyrich molecules, considered as excellent alternative to liquid transport fuels and chemicals.

The engineering strategies in microbes could be divided into three parts, i.e. traditional engineering, rational metabolic engineering and combinatorial engineering. Traditional engineering approach through UV/chemical mutagenesis or evolutionary engineering generate strains with desired phenotypes. This approach is straightforward and capable to generate new traits but is usually time-consuming and labor intensive. Rational metabolic engineering is usually based on the regulation of specific metabolic 
pathways, which requires detailed metabolic, genetic, stoichiometric, kinetic, and regulatory knowledge. In combinatorial engineering, multiple genes expression level could be modified spontaneously to elicit altered strain phenotypes. These engineering strategies have attracted much attention in strain engineering in recent years since most of the detailed mechanism remain uncharacterized. Especially, as one of combinatorial engineering approaches, global transcription machinery engineering (gTME) is capable to reprogram cellular transcription profile to elicit valuable traits through fine-tuning of global transcription machinery components such as transcription factors (TFs), TATAbinding protein (Spt15), zinc finger-containing artificial transcription factors, etc. Our group has successfully improved E. coli tolerance towards ethanol and other stressors by engineering its global regulator cAMP receptor protein (CRP) (Chong, Huang2 et al. 2013).

Based our experience in gTME, one of the work in this thesis is focused on engineering eukaryotic microbe and exploiting different engineering target based on eukaryotic transcription machinery. In eukaryotic system, apart from TFs, RNA polymerase II (RNAP II) plays a central role in synthesizing all mRNAs. Among the twelve subunits of RNAP II, Rpb7 plays important roles at various stages of genes expression, such as interaction with RNAP II transcription apparatus \& nascent RNA transcripts and participation in mRNA export and decay. The multifunction of Rpb7 suggests the possibility of mutating Rpb7 to elicit cellular transcription profile change for desired phenotypes in yeast.

Another research in this thesis is focused on improving fatty acids production in $S$. 
cerevisiae. The fatty acids synthesis pathway in yeast is well studied. One major problem for the limited production of fatty acids is that the supply of its precursor malonyl-CoA in cytosol is quite low. Since malonyl-CoA is initiated by the conversion of acetyl-CoA, the most common metabolic strategies were to direct the glycolytic fluxes to acetyl-CoA biosynthesis, which mainly focused on the "acetyl-" part. Pantothenate kinase (PanK) catalyzes the phosphorylation of pantothenate, which is the rate-limiting reaction for CoA synthesis. Our lab has successfully increased acetyl-CoA level in yeast cytosol by 24-fold through enhancing "acetyl-" part and the "-CoA" part simultaneously, providing a solid acetyl-CoA supply platform (Liu, Zhang et al. 2017). In addition to the acetyl-CoA carboxylase pathway, the malonyl-CoA biosynthesis pathway is an alternative for the accumulation of cytosolic malonyl-CoA. The MatB gene from the bacteria Rhizobium leguminosarium encodes a malonyl-CoA synthetase which directly catalyzes the formation of the malonyl-CoA from malonate and CoA. In addition, a dicarboxylic acid plasma membrane transporter MAE1 from Schizosaccharomyces pombe that could uptake exogenous malonic acid was introduced into $S$. cerevisiae to increase the cytosolic malonate. Based on the two pathways discussed above, one work of this study is to combine the acetyl-CoA carboxylase pathway and malonyl-CoA synthetase pathway to enhance the malonyl-CoA supply for improving fatty acids synthesis.

\section{The specific aims of this study are as follows:}

1. To improve $S$. cerevisiae ethanol tolerance and production via random mutagenesis of Rpb7. Error-prone PCR technology has been applied to generate random 
mutagenesis library that was transformed into yeast cells later on. The isolated mutants have been identified based on their growth under ethanol stress. The ethanol production and its glucose consumption of the ethanol-tolerant mutant were measured. The cellular transcription profile change would be revealed by the DNA microarray and qRT-PCR.

2. To study bioalcohol tolerance mechanism, its relationship with production and recent engineering strategies. The mechanism would be discussed from genome \& epigenome, transcription, translation, metabolism, and plasma membrane aspects. The corresponding mechanism-inspired engineering approaches to improve bioalcohol tolerance would also be reviewed. In addition, combinatorial engineering approaches, including genome shuffling, random overexpression/deletion screening, global transcription machinery engineering, and classical strain engineering approach of adaptive evolution \& hybridization have also been discussed.

3. To improve fatty acids production in $S$. cerevisiae via increasing precursor malonyl-CoA supply. The acetyl-CoA carboxylase pathway (overexpression of PanK, $A L D 6, \operatorname{Se} A c s^{L 641 P}$ and $A C C 1^{\mathrm{S} 659 \mathrm{~A}, \mathrm{~S} 1157 \mathrm{~A}}$ ) and malonyl-CoA synthetase pathway (introduction of $M A E 1$ and $M a t B$ ) had been introduced into S. cerevisiae. The fatty acids production and composition had been detected by GC-MS.

In this dissertation, there will be six chapters. Chapter 1 gives a general introduction of the whole project and presents the specific aims. Chapter 2 includes the background and literature review of strain engineering. Chapter 3 is an accepted article in Journal 
of Biotechnology for Biofuel, which describes the results of engineering $\mathrm{Rpb} 7$ to improve S. cerevisiae ethanol tolerance and production. Chapter 4 reviews the bioalcohol tolerance mechanism in $S$. cerevisiae, as well as its engineering strategies. Chapter 5 reports the results of improving fatty acids production through enhancing the supply of malonyl-CoA precursor in S. cerevisiae. The conclusions are shown in Chapter 6. 


\section{Chapter 2. Background}

In this chapter, Saccharomyces cerevisae (S. cerevisiae) application for biofuel production as well as current different engineering strategies will be reviewed. Additionally, the RNA polymerase II subunit Rpb7 in S. cerevisiae will be discussed based on its structure and transcriptional function. Last but not least, the metabolic engineering approaches to increase fatty acid production in $S$. cerevisiae will be reviewed from different engineering strategies.

\subsection{Biofuel and its application}

The increasing demand of sustainable energy requires exploiting new technologies, which are more environment friendly, economic profitable and large-scale available. Biofuels resource energy, accounted as one of the most promising renewable energy sources, has its advantages in the global spread, transportation and storage (Demirbas and Ayhan 2008). First generation biofuels are characterized by their ability to be mixed with fossil fuels existing in current combustion engines, including biodiesels, bioalcohols, and biogas (Naik, Goud et al. 2010). In contrast of the first-generation biofuels that normally utilize food as carbon source, second-generation biofuels are produced from non-food biomass of plant waste such as ligocellulose. To confront with the approaching challenges of energy security crisis and the goal of the Kyoto Protocol to lower $\mathrm{CO}_{2}$ emission, International Energy Agency (IEA) has developed a set of ambitious projects to accelerate the 2 nd-generation biofuels production to meet $26 \%$ of the overall transport fuel demand in 2050 and to reduce the biofuels cost as low as USD 70/bbl (Demirbas and Ayhan 2008, Sims, Mabee et al. 2010). However, through 
accounting the life-cycle of 2nd-generation biofuels, high agricultural input provides limited benefit under the current economic and environmental situation (Hill, Nelson et al. 2006). To address this dilemma, much more efforts are required to improve the conversion efficiency from biomass into biofuels, leading to extensive studies in fermentation process and microorganism engineering in the recent years.

\subsection{Saccharomyces cerevisae as host strain in biofuel production}

Among all the microbial organism for biofuels production, E. coli and S. cerevisiae are the most popular host strains due to the following advantages: (i) high capability to accept foreign genes; (ii) sufficient biological tools to manipulate the genes expression and cell performance; (iii) well-known background in metabolic pathways and genome information (Fischer, Klein-Marcuschamer et al. 2008). However, compared with $E$. coli, S. cerevisiae has special advantages, such as post-translational modifications, capacity of complex enzyme expression, less possibility of phage contamination and high resistance towards toxic inhibitors and products (Nielsen and Jewett 2008, Chen, Daviet et al. 2013, Lian, Si et al. 2014). In addition, S. cerevisiae has long been adopted as a superb ethanol producer and exploited as a potential candidate workhorse for other biofuels production, including 1-butanol (Steen, Chan et al. 2008), isobutanol (Chen, Nielsen et al. 2011, Brat, Weber et al. 2012), sesquiterpenoids farnesene (Ubersax and Platt 2010), bisabolene (Peralta-Yahya, Ouellet et al. 2011), amorphadiene (Paradise, Kirby et al. 2008) and fatty acid ethyl esters (FAEEs) (biodiesel) (Shi, Chen et al. 2014).

\subsection{Strain engineering}

In order to obtain desired strain phenotypes for industrial application, e.g. tolerance 
towards extreme conditions (high temperature, low $\mathrm{pH}$ etc.), high productivity of the desired products, various engineering strategies have been discovered and investigated during the latest decades, including classical strain engineering, metabolic engineering and combinatorial engineering. Classical strain engineering that applies evolutionary engineering or UV/chemical mutagenesis followed by screening process has long been treated as the gold standards for industrial strain improvement (Patnaik 2008). However, the long screening period and complex strain selection process are often considered as its drawback.

On the other hand, through targeting critical factors or pathways that are related to the phenotypes, such as specific promoter sequences, regulators, intracellular enzymes, transporters, membrane components, metabolic engineering is conducted by adopting different recombinant DNA techniques that allow genetic manipulation (Park and Lee 2008). This rational engineering strategy highly depends on the existing knowledge of genetic background, genes expression regulatory networks, metabolic pathway and kinetics, stoichiometric, etc. (Santos and Stephanopoulos 2008). Hence, its application is limited when the engineered phenotype is under multi-genetic regulation or the genetic background is unknown.

To deal with the problems and limitations in classical engineering and metabolic engineering, inverse metabolic engineering (IME) emerged in the recent years following a contrary schematic flow to the conventional metabolic engineering, which could be briefly described as: first, identifying an instructive phenotype as well as the specific determining genetic or environmental factors conferring that phenotype; 
second, libraries consist of these random factors (usually as genetic variants) are transformed to another strains or organisms and cells with desired phenotypes are selected; finally, inserted factors in charge of the superior property are characterized and studied (Bailey, Sburlati et al. 2002, Wang, Ghaderi et al. 2014). Combinatorial engineering is generated as an engineering tool derived from IME, which includes random overexpression/knockout, genome shuffling, ribosome engineering, transcriptional engineering. The information acquired from combinatorial engineering and classical engineering is helpful to understand metabolic and regulatory knowledge of strains as well.

\subsubsection{Classical Strain Engineering}

Since no prior knowledge is required in the metabolic pathway and genetic information of the host strain, classical engineering has always been applied in optimizing industrial strain for a long period. Classical strain engineering is easy to conduct and capable of eliciting new phenotypes. It includes evolutionary engineering and UV/Chemical mutagenesis, both of which require repeated rounds of genetic diversity generation and phenotypes selection (Petri and Schmidt-Dannert 2004). Figure 1 illustrates the general schematic workflow of the classical engineering. Here, the repeated subculture and mutagenesis is intended to generate or introduce random mutation, e.g. strand breakage, transversion, addition, deletion or base substitutions, to host strain genome (Patnaik 2008) and the selection process always varies to mimic natural selection procedure to evolve strains. There two principles that may largely determine the success of the engineering: (i) moderate degree of the mutagen 
(UV/chemical mutagens); (ii) appropriate selection pressure towards the culture (Cakar,

Seker et al. 2005). However, since it usually takes hundreds of generations to evolve the ideal strains, classical strain engineering is quite time consuming and labour intensive, considered as its main disadvantages.

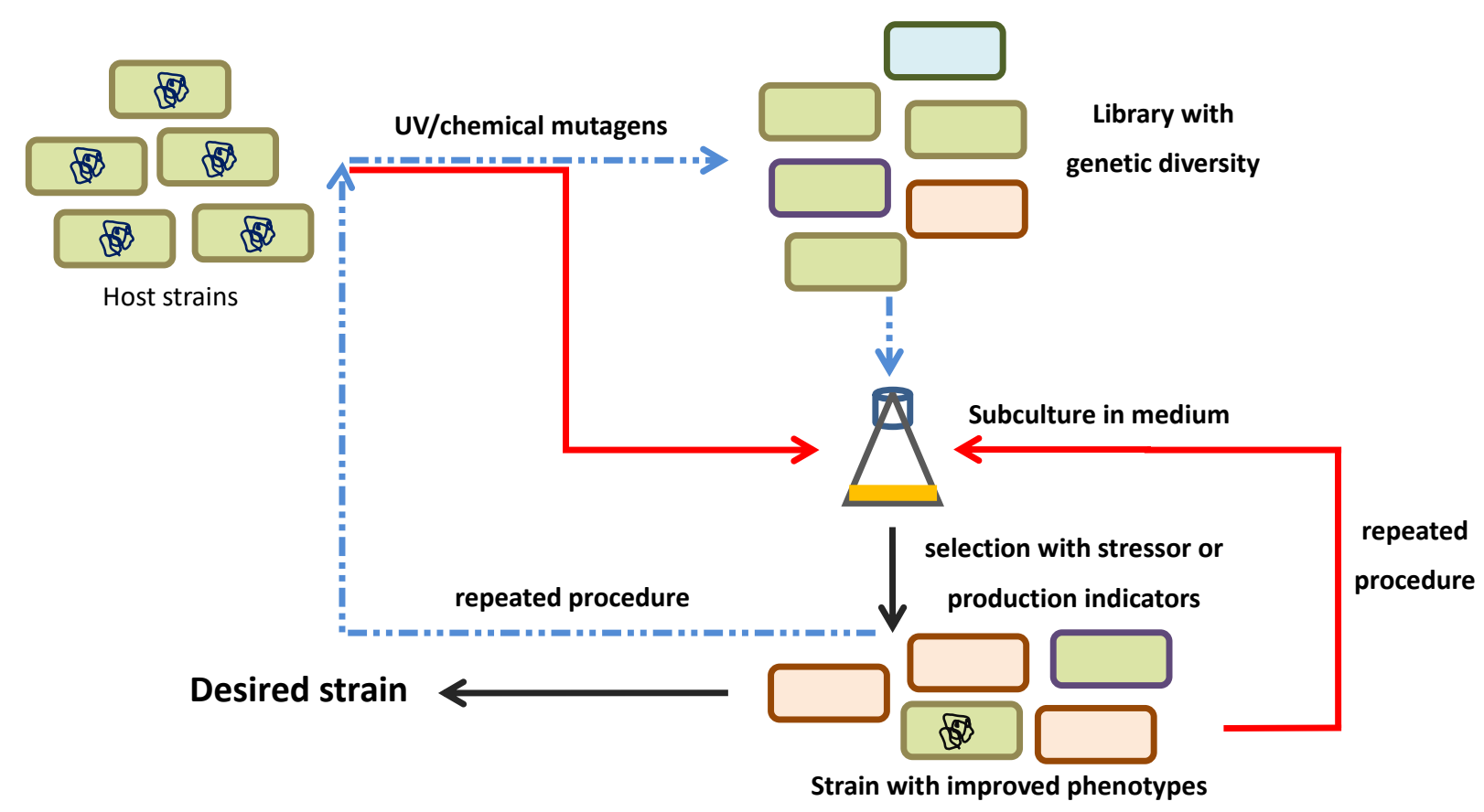

Figure 1. Schematic diagram showing the general workflow of traditional strain engineering. The

selection process is continued and repeated until mutants with desired phenotypes are selected. Blue dashed line: the process of random mutagenesis using UV/chemical mutagens; red solid line: the process of evolutionary engineering; black solid line: selection of strains with better phenotype.

\subsubsection{Evolutionary engineering}

In the repetitive breeding evolution, microbes are subjected to repeated batch cultivation or long-term chemostat cultivation in the presence of certain stressors. During the culturing procedure, spontaneous genetic change occurs and adaptive monoclonal population survives and accumulates in a stressful environment (Hersh, 
Ponder et al. 2004). Finally, the accumulated variants will then be enriched and dominate in the culture after multiple rounds of subculture (Figure 1) (Cakar, TuranliYildiz et al. 2012).

In E. coli, a isobutanol-tolerant strain SA481 was isolated after a total of 45 rounds of subculture in LB medium containing isobutanol. Its survival rates were 13- and 5fold higher than the control in $6 \mathrm{~g} / \mathrm{L}$ and $8 \mathrm{~g} / \mathrm{L}$ isobutanol, respectively (Atsumi, Wu et al. 2010). To increase the consumption of xylose in S. cerevisiae, parent yeast strain TMB3001 was subjected to long-term chemostat cultivation by over four hundred generations to acquire variants that could utilize xylose as carbon source (Sonderegger and Sauer 2003).

In addition, formation of hybrids derived from industrial and laboratory stains is another natural evolution scheme in pursue of increasing genetic variability. Through this method, an optimal S. cerevisiae was selected that is able to grow under lethal ethanol condition (16\% (v/v)) (Jiménez and Benítez 1988).

\subsubsection{UV/Chemical mutagenesis}

The objective to use UV/Chemical mutagen for strain engineering is to increase the mutation possibility in host strain genome. The strain bearing the beneficial mutations will display better phenotype during the screening process and finally be selected. Here, UV ray, N-methyl-N'-nitro-N-nitrosoguanidine (NTG) and ethyl methanesulfonate (EMS) are the most commonly used mutagens (Biot-Pelletier and Martin 2014). For examples, NTG was applied to increase mutation in E. coli for improving 3-Methyl-1-butanol production (Connor, Cann et al. 2010) and acid 
tolerance in Lactobacillus (Patnaik, Louie et al. 2002). Through employing EMS, fibrinolytic protease productivity increased up to $14.78 \mathrm{U} / \mathrm{mL} / \mathrm{min}$ in Bacillus cereus GD 55 (Raju and Divakar 2013). UV-C mutagenesis was also applied for improvement of ethanol tolerance of $S$. cerevisiae NR1 for enhancing ethanol production (Thammasittirong, Thirasaktana et al. 2013).

\subsubsection{Metabolic engineering}

In metabolic engineering, the engineering strategy is highly based on the existing knowledge of the host strains and the current gene manipulation technologies. Commonly, the engineering approaches include expression modification, deletion or introduction of enzymes, transporters or regulatory proteins. Especially, heterogeneous expression of a specific pathway for producing non-natural compounds in the host cell is quite common in metabolic engineering. For example, 1-butanol synthetic pathway was heterogeneously introduced in E. coli for 1-butanol production (Atsumi, Cann et al. 2008). In S. cerevisiae, n-butanol biosynthetic pathway from Clostridium was expressed and the $n$-butanol production in yeast reached $2.5 \mathrm{mg} / \mathrm{L}$ after the fermentation (Steen, Chan et al. 2008). Recently, Guo et al. have successfully produced 1hexadecanol over $1.2 \mathrm{~g} / \mathrm{L}$ using xylose as the sole carbon source through introducing fungal xylose utilization pathway (Guo, Sheng et al. 2016). Metabolic engineering strategy is direct and it could introduce exogenous phenotypes from other species. However, it is based on the comprehensive knowledge of the metabolic pathway of the products and strain background. In addition, for the phenotypes with multiple genes control, it is hard to modify all the genes expression simultaneously. 


\subsubsection{Combinatorial engineering}

Even though rational modifications of phenotypes-relevant genes or factors are straightforward, they still have limits since: i) systematic mechanism is not completely clarified; ii) microbial traits are normally under polygenetic control and the result of thousands of gene interaction and gene products. A recent global genotype-phenotype map (GPM) indicates that eukaryotic traits are more likely influenced by small-effect genes such as distal genes (Ho and Zhang 2014). In addition, to address the fundamental complexity in eliciting improved phenotypes, engineering strategies require spontaneous modulation of multiple genes expression and metabolic pathway shifts (Bailey 1999). Following the principle of inverse metabolic engineering, which firstly proposed by James E. Bailey (Bailey, Sburlati et al. 2002), combinatorial engineering strategies have started to attract attentions in recent years as most of the detailed mechanism information remains uncharacterized. However, combinatorial engineering depends on high-throughput mutant screening and isolation approaches to identify the strains with improved phenotypes in large library.

\subsubsection{Random overexpression/knockout}

Successful random overexpression/deletion approaches in search of optimalphenotype isolates and associated genes rely on: a priori knowledge on phenotypes linked mechanism for predicting the overexpression/deleltion targets; potent recombinant DNA technology in order to construct random genetic variants libraries with large capacity; high-throughput screening approach to select variants with substantial phenotype change (Santos and Stephanopoulos 2008, Wang, Ghaderi et al. 
2014).

The transformants libraries can stem from endogenous or heterogenous genome. For example, through transformation of $S$. cerevisiae autologous genomic library, competitive variants carrying INO1, DOG1, HAL1 or truncated MSN2 were selected by showing improved tolerance towards ethanol and isobutanol (Honga, Leea et al. 2010). In addition, two transformant yeast strains overexpressing $A D H 1$ and $H X T 1$ from the overexpression library pool demonstrated improved fermentative efficiency by $80.33 \%$ to $84.57 \%$ respectively (Gutiérrez-Lomelí, Torres-Guzmán et al. 2008). In regards of random knockout, transposon mutagenesis, by means of integrating antibiotic/amino acid synthesis maker cassette into the genome, has been applied to generate genomescale knockout library. The random knockout technology has been applied to improve L-tyrosine final production (71\% higher) in E. coli (Santos and Stephanopoulos 2008). In addition, in contrast of null mutation, recent emerging techniques in control of gene expression such as clustered regularly interspaced short palindromic repeats (CRISPR) sequence-specific control or synthetic small regulatory RNAs are more flexibleregulated approaches to control global expression in purpose of generating better industry-prone strains (Na, Yoo et al. 2013, Qi, Larson et al. 2013).

\subsubsection{Genome shuffling}

Genome shuffling combined with artificial protoplasts fusion is a powerful and rapid way to introduce mutation in strains. This approach is to achieve larger genetic diversity, i.e. to expand genomic background to elicit desired phenotype. Various attempts had been made to induce genetic variation, including pretreatment of 
protoplast by ultraviolet irradiation (Shi, Wang et al. 2009), high-energy pulse electron beam (HEPE) (Zhang, Xiao et al. 2012), chemical mutagens (Liu, Ding et al. 2011), and error-prone genome amplification (Luhe, Tan et al. 2011). Besides, homologous genome recombination can be achieved by adopting native strain (Zheng, Chen et al. 2014) or different donors (Shi, Wang et al. 2012). Whole genome shuffling has been successfully applied to improve ethanol yield by $10.96 \%$ in S. cerevisiae (Hou 2010). In addition, genome shuffling with UV-light improved $S$. cerevisiae tolerance towards spent sulfite liquor from pulp mills, which is considered as low-cost feedstocks for fermentation (Pinel, D'Aoust et al. 2011). In Lactobacillus rhamnosus, the L-lactic acid productivity was increased by $26.5 \%$, up to $5.77 \mathrm{~g} / \mathrm{L} / \mathrm{h}$ by genome shuffling (Wang, Li et al. 2007). However, this approach was questionable as multiple rounds of genome shuffling may induce the loss of existing beneficial properties since the chance of acquiring new improved mutations equals to the chance of losing existing advantageous genomic fragments.

\subsubsection{Ribosome engineering}

Ribosome engineering focuses on activation of dormant genes to fully elicit cellular function. Some antibiotics, e.g. streptomycin, gentamicin, kanamycin and chloramphenicol, could attack ribosome, causing random mutations in $r p o L$ (encoding ribosomal protein S12) and activating dormant genes (Ochi 2007). Hence, mutants with different drug-resistance can be isolated and selected from the drug-containing medium. Ribosome engineering is potential for multiple application such as strain improvement, screening of novel metabolites, plant breeding, cell-free translation systems, and the 
treatment of tuberculosis (Chi 2007).

In fact, ribosome engineering was widely used as a rational approach to improve antibiotic production in Streptomyces (Olano, Lombo et al. 2008, Wang, Hosaka et al. 2008, Lv, Jin et al. 2013). It has also been used to improve $\alpha$-Amylase production $(\sim 40 \%$ higher than the parent strain) in Bacillus subtilis 168 (Kurosawa, Hosaka et al. 2006) and xylanase production ( $14 \%$ higher) in Streptomyces viridochromogenes (Liu, Zhao et al. 2013).

\subsubsection{Global transcription machinery engineering (gTME)}

In the light that most phenotypes are the result of global transcription alteration in microbes, the potential of engineering strategies that merely rest on single gene modification by rational or random approach would be highly limited. The improvement of adaptive complex traits required simultaneous/induced modification in the expression of hundreds of genes. Global transcription machinery engineering (gTME) is able to reprogram global genes transcription to elicit valuable cellular phenotypes for industrial application through fine-tuning components of global transcription machinery (Alper and Stephanopoulos 2007). The components selected ought to be key proteins regulating the global transcriptome such as critical transcription factors, artificial transcription factors, and factors affecting the preference of RNA polymerase recruitment (Park, Lee et al. 2003, Alper, Moxley et al. 2006, Chong, Geng et al. 2014) (Figure 2). To state the advantages of gTME in strains breeding, one research group in MIT (Klein-Marcuschamer and Stephanopoulos 2008) compared the introduced phenotypic diversity in a quantitative platform between the 
gTME approach based library (which introduce random mutation in RNA synthesis initiation factor $\sigma$-factor (rpoD) of Lactobacillus plantarum) and the conventional chemical mutagenesis by NTG based library. The higher distribution of the average phenotypic distance from gTME generated library implied its superior efficiency to endow organism phenotype variation, demonstrating that the trans-acting regulation could lead to a high degree of pleiotropy. In S. cerevisiae, the transcription factor Spt15 has been successfully engineered to improve ethanol and production (Alper, Moxley et al. 2006). The transcription factor Taf 25 has also been engineered to improve yeast oxidative tolerance towards $3.5 \mathrm{mM} \mathrm{H}_{2} \mathrm{O}_{2}$ (Zhao, Li et al. 2014).

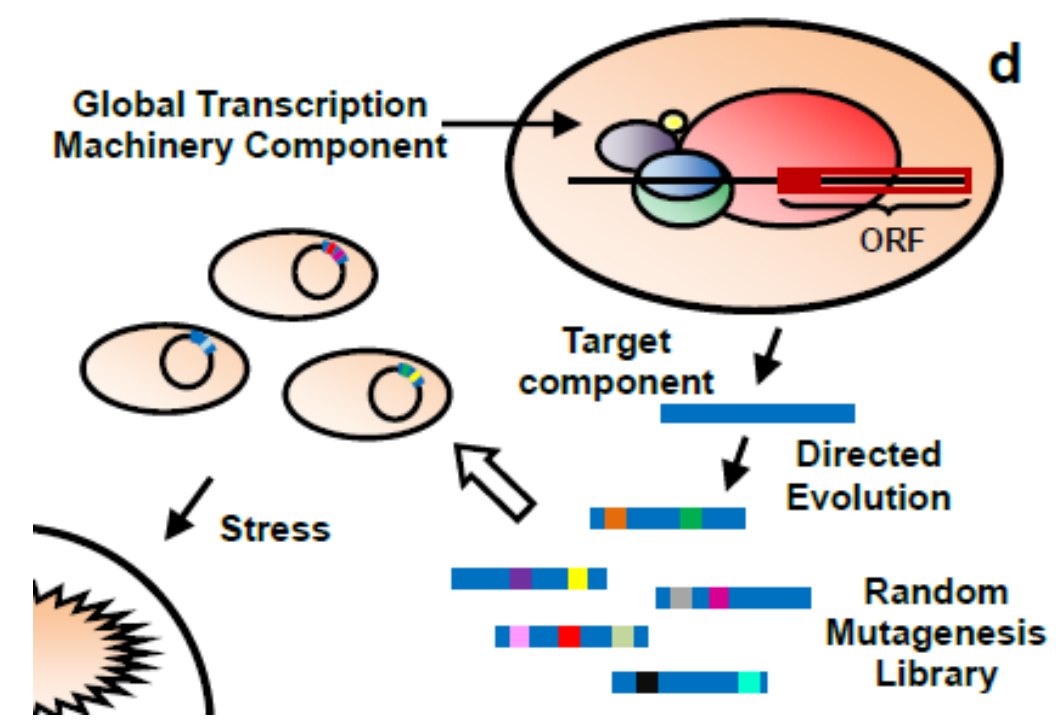

Figure 2 The workflow of gTME. The global transcription machinery component is selected and its ORF is chosen as the engineering target. Through directed evolution, random mutagenesis is introduced into the target. After transforming the mutant library into the host cells, the mutant with better performance is isolated.

There's also some challenges in gTME. In fact, to find out a high-throughput screening method is always a bottleneck for gTME. A recent microfluidic detecting 
system under the fluorescence-activated cell sorting (FACS) technique has proven to be the one of the most efficient screening approaches so far for application in different phenotypes strains selection (Wang, Ghaderi et al. 2014).

\subsection{RNA polymerase II subunit Rpb7}

In eukaryotic cells, RNA polymerase II plays a vital role in genes transcription. As the core enzyme in mRNA synthesis, RNAP II, affects promoter affinity in mRNA synthesis regardless of TFs and is also involved in post-initiation regulation including mRNA elongation, proofreading, stabilization and etc. (Cramer 2004, Sainsbury, Bernecky et al. 2015). Therefore, larger degree of perturbation in global expression may be obtained through engineering RNAP II directly. Among the twelve yeast RNAP II subunits, Rpb7 draws much attention based on its multiple function in mRNA synthesis and processing. $\mathrm{Rpb} 7$ is located at a specific region that is near to the transcript-exit groove and the C-terminal domain (CTD) of Rpb1 (Choder 2004). It participates in interaction with processing factors of RNAP II transcription apparatus and the nascent RNA transcripts, including stabilizing RNAP II structure and binding ssRNA (Woychik and Hampsey 2002, Jasiak, Hartmann et al. 2008). In addition, RPB7 also forms Rpb4/7 heterodimer with Rpb4 to shuttle between nucleus and cytoplasm, serving as "mRNA coordiators" in various stages of mRNA lifecycle, e.g. mRNA export and decay (Lotan, Goler-Baron et al. 2007, Harel-Sharvit, Eldad et al. 2010). Figure 3 illustrates the multiple function of Rpb7 in genes transcription and expression. All these facts indicate that RPB7 have a wide impact on gene expression in multiple levels. 


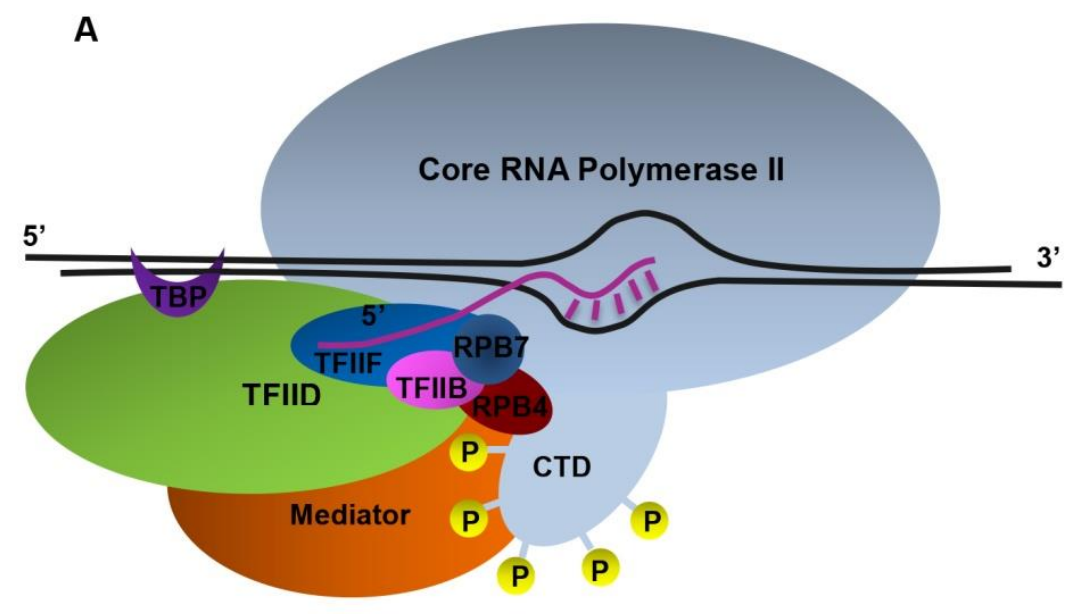

B

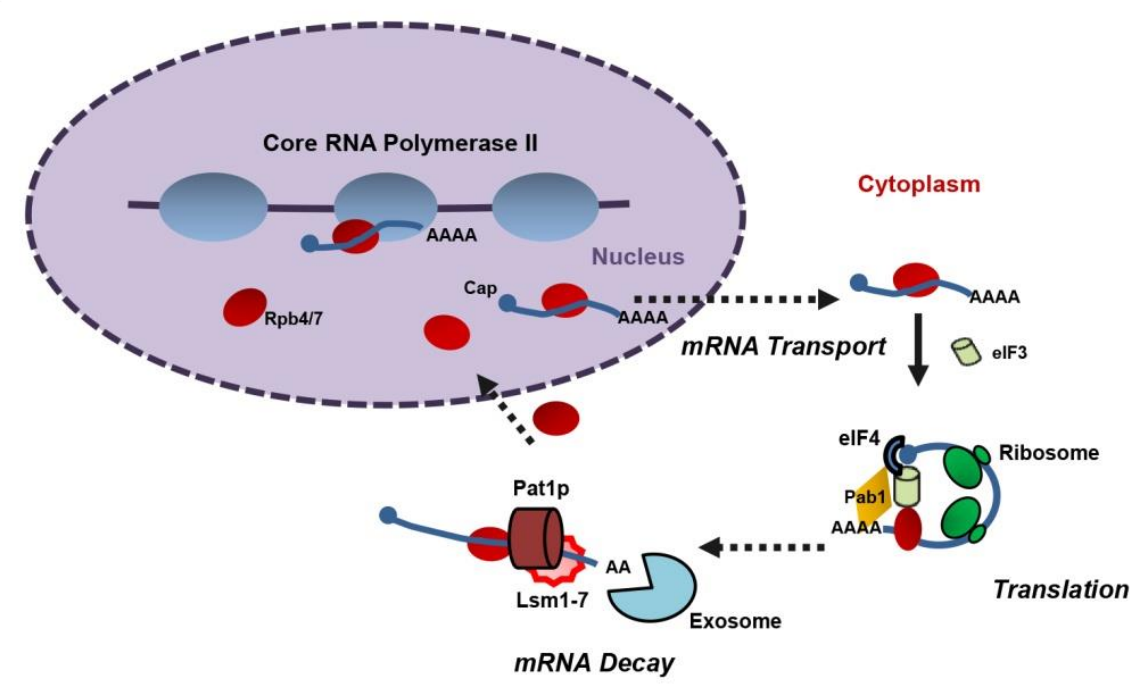

Figure 3 Models of Rpb7 multifunction in gene expression. Rpb7 usually fulfills its function by forming sub-complex with Rpb4, but the major role of Rpb4 is to augment the interaction of Rpb7 with Pol II (Sheffer et al., 1999). (A) In the transcription initiation complex, the Rpb4/7 sub-complex is situated closed to the nascent-transcript-exit groove and adjacent to Rpb1 C-terminal domain (CTD) linker region (Choder, 2004), and it is also located near general transcription factor TFIIB and can physically interact with TFIIF (Shpakovski et al., 2000). (B) The role of Rpb4/7 in posttranscription regulation, including mRNA export, translation and mRNA decay (Harel-Sharvit et al., 2010). 


\subsection{Metabolic engineering approaches to increase fatty acid production}

\subsubsection{Fatty acid and its derivatives as biofuels}

Fatty acids or their activated forms, fatty acyl-CoAs and fatty acyl-ACPs, are energy-rich molecules, considered as important precursors for various advanced biofuels such as free fatty acids (FFAs), fatty alcohols (FALs), fatty acid ethyl esters (FAEEs), and alkanes (Lian and Zhao 2015). These compounds could be alternatives of fossil fuels since they have similar properties of current transportation fuels (Zhang, Rodriguez et al. 2011). The microbial conversion of renewable feedstock into these fuels has been widely investigated in recent years. Compared to other microbial hosts, S. cerevisiae offers many advantages for large-scale biofuels production due to its well understood genetic background, established genetic tools, post-transcription modification, short generation of cultivation time, high tolerance against toxic organic solvent and products (Hong and Nielsen 2012). Therefore, S. cerevisiae is an ideal cell factory platform for metabolic engineering for fatty acid production.

\subsubsection{Fatty acids synthesis in Saccharomyces cerevisae}

Fatty acids metabolism and regulation have been well-characterized in S. cerevisiae. Fatty acid biosynthesis (FAB) is initiated by the conversion of acetyl-CoA to malonylCoA via the enzyme of acetyl-CoA carboxylase in the cytosol of yeast cells. The malonyl-CoA is then transferred to fatty acid synthases (FASs) complex for elongation. Finally, deno novo synthesized fatty-CoAs could be converted to desired products, such as FFAs, FALs, FAEEs by the corresponding terminal enzymes (Nielsen and Keasling 2016). However, the supply of precursors (acetyl-CoA and malonyl-CoA) are very 
limited in yeast cells and the FAS systems are tightly regulated, finally causing low level of natural fatty acids production in yeast (van Roermund, Waterham et al. 2003). Especially, the limited supplies of cytosolic acetyl-CoA and malonyl-CoA are the major bottlenecks for de novo biosynthesis of fatty acids. Therefore, extensive metabolic engineering strategies have been focused on increasing the glycolytic fluxes to acetylCoA as well as the malonyl-CoA synthesis in yeast.

\subsubsection{Fatty acids production through overproducing acetyl-CoA}

Acetyl-CoA is the central metabolite and the precursor of a wide range of bioproducts. These products are mostly synthesized in cytosol. The acetyl-CoA generated in mitochondria from pyruvate by pyruvate dehydrogenase (PDH) complex requires carnitine/acetyl-carnitine shuttle to be transported out of mitochondria (Strijbis and Distel 2010, Chen, Daviet et al. 2013). The engineering strategies intended to direct the metabolic flux to acetyl-CoA biosynthesis are discussed as followed (Figure 4). 


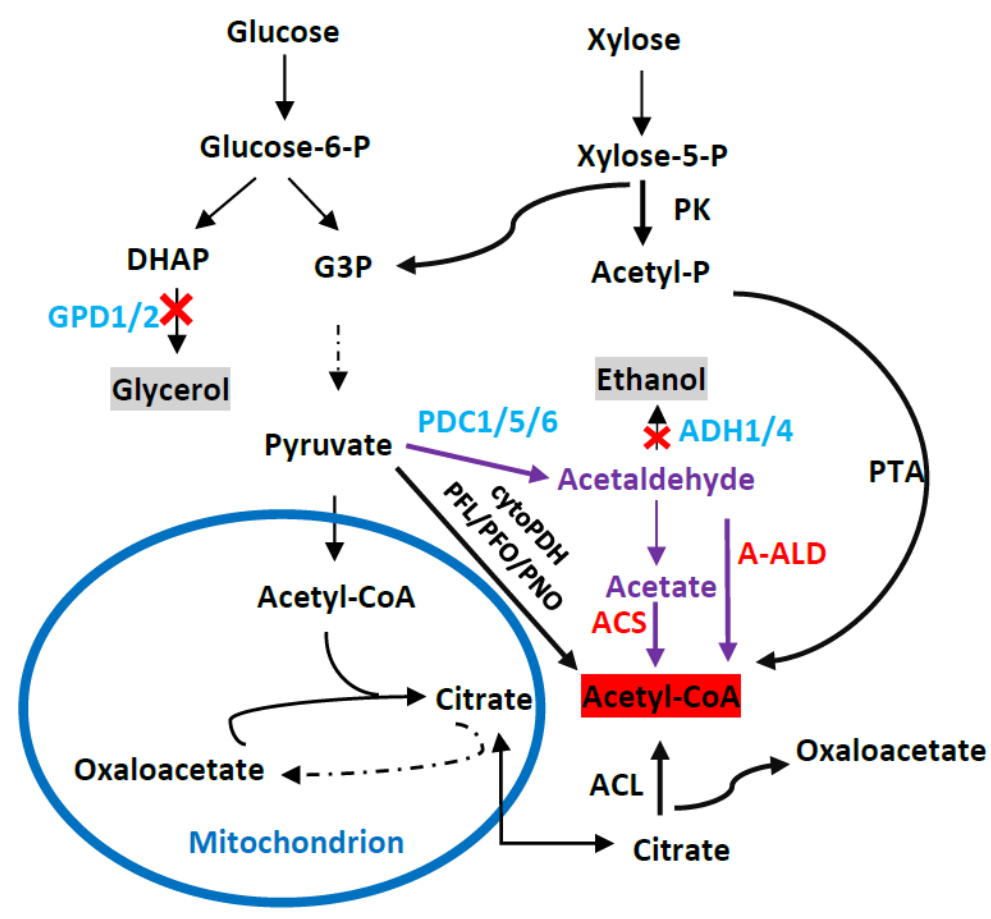

Figure 4 Design and construction of acetyl-CoA overproducing yeast strains. The PDH bypass engineered in this study is marked in purple.

\subsubsection{Redirect the glycolytic flux from ethanol synthesis to acetyl-CoA synthesis}

Since most of the glycolytic fluxes go to the ethanol formation due to the Crabtree effect, the central metabolism of yeast was modified to limit ethanol production and redirect the metabolic fluxes to actyl-CoA synthesis. The alcohol is synthesized by the alcohol dehydrogenases (ADHs). Through knock-out of major ADHs in the cytosol $(\triangle A D H 1-\triangle A D H 4)$, acetyl-CoA level increased by 2 -fold and the production of $n$ butanol derived from acetyl-CoA also increased by 4-fold (Lian, Si et al. 2014). In addition, the deletion of $A D H 1$ improved the fatty acids of $S$. cerevisiae up to 1.9-fold (Li, Guo et al. 2014).

\subsubsection{Inactivate acetyl-CoA consuming pathways}

The strategy intended to eliminate the aceyl-CoA consumption pathway has been focused on glycoxylate shunt, which contributes to the consumption and transport of 
cytosolic aceyl-CoA. MLS1 and CIT2 encode malate synthase and citrate synthase respectively, which participate in glyoxylate shunt. After knocking out these two genes, the production of acetyol-CoA derivatives such as $\alpha$-santalene, 3-hydroxypropionic acid (3-HP) and $n$-butanol increased by 2.27 -fold, 1.20 -fold and 1.36 -fold respectively (Chen, Daviet et al. 2013, Chen, Daviet et al. 2013, Wang, Chen et al. 2014).

\subsubsection{Engineering PDH-bypass pathway}

PDH bypass is composed of pyruvate decarboxylase (PDC), acetaldehyde dehydrogenase (ACDH, such as Ald6), and acetyl-CoA synthetase (ACS) (Figure 4). In the PDH bypass, the acetaldehyde generated from pyruvates by PDC could be converted into acetate by $\mathrm{ACDH}$, or to ethanol by alcohol dehydrogenase (ADH). Then, acetate would be converted into acetyl-CoA by ACS. In yeast cells, the insufficient acetate supply was found to be one of the reasons for limited acetyl-CoA biosynthesis as well (Remize, Andrieu et al. 2000). Therefore, acetaldehyde dehydrogenase (ADH2) and/or aldehyde dehydrogenase (ALD6) and $\mathrm{Se} A c s^{L 641 P}$ from Salmonella enteric have been overexpressed to push the metabolites to acetate and then acetyl-CoA synthesis. The engineered strains by this approach have 1.75 -fold increase in production of $\alpha$ santalene (Chen, Daviet et al. 2013), 1.22-fold increase in amorphadiene (Shiba, Paradise et al. 2007) and 1.50-fold increase in production of 3-HP (Chen, Daviet et al. 2013).

\subsubsection{ATP-dependent citrate lyase (ACL)}

In oleaginous fungi, there is another source of cytosol acetyl-CoA production from the excess of citrate. The excessive citrate is exported to cytosol from mitochondria via 
citrate transport protein and then cleaved by ATP-citrate lyase (ACL), an enzyme existed in oleaginous yeasts. ACLs from mammalian cells have been introduced and functionally expressed in $S$. cerevisiae to increase fatty acids production by 1.17 -fold (Tang, Feng et al. 2013) (Tang and Chen 2014). Similarly, ACL from oleaginous yeasts was expressed in $S$. cerevisiae and $n$-butanol showed about 2 -fold increase after the fermentation (Lian, Si et al. 2014).

\subsubsection{Pyruvate dehydrogenase (PDH) pathway}

The most efficient route to synthesize acetyl-CoA is through the oxidative degradation of pyruvate by the PDH catalysis. However, this pathway can only be found in prokaryotic microbes and eukaryotic organelles. Therefore, some engineering strategy has been focused on the introduction and functional expression of the mitochondrial PDH into the cytosol of yeast. PDH from E. coli was also assembled in yeast for the similar purpose. By introducing cytosolic $P D H s$ into the strain with $A D H 1$ and $A D H 4$ deletion, the acetyl-CoA density and $n$-butanol production increased by about 1.38 and 3.01 -fold respectively (Lian, Si et al. 2014).

\subsubsection{Pantothenate kinase pathway}

Most of the studies for improving acetyl-CoA level in S. cerevisiae have been focused on engineering the "acetyl-" part of acetyl-CoA, but there are no reports on engineering the "-CoA" part so far. Pantothenate kinase (PanK) that catalyzes the phosphorylation of pantothenate is considered to be the rate-limiting enzyme for CoA synthesis (Olzhausen, Schubbe et al. 2009). It has been reported that the overexpression of $m P a n K 1 \beta$ (an isoform of PanK) in mammalian cells could lead to 13-fold increase 
in the level of intracellular CoA (Rock, Calder et al. 2000). Through overexpressing PanK in E. coli, the intracellular CoA and acetyl-CoA level have 10 and 5-fold increase respectively (Vadali, Bennett et al. 2004). Based on this strategy, our group has successfully introduced PanK encoding endogenous gene $C A B 1$ to increase acetyl-CoA level by over 3-fold in S. cerevisiae.

\subsubsection{Fatty acids production through engineering the step from acetyl-CoA to malonyl-CoA}

The conversion of acetyl-CoA to malonyl-CoA is catalyzed by acetyol-CoA carboxylase (ACC). This step is critical and rate-limiting for fatty acid biosynthesis. Engineering approaches including overexpression of ACC1 and improvement of the ACC1 catalytic efficiency have been performed.

Overexpression of ACC1 has led to improved malonyl-CoA level. However, the malonyl-CoA derived molecules (e.g. fatty acid production) only increased to a limited extent, i.e. less than 2-fold improvement in most cases through simple ACC1 overexpression (Wattanachaisaereekul, Lantz et al. 2008, Shin, Jung et al. 2012, Runguphan and Keasling 2014, Shi, Chen et al. 2014, Wang, Chen et al. 2014, Feng, Lian et al. 2015). These results indicate the complicated regulation of ACC1 enzyme. The activity of ACC1 was post-translationally regulated by a protein kinase SNF1. The phosphorylated ACC1 by SNF1 would lost its catalytic activity. Ser659 and Ser1157 were identified as the two putative phosphorylation sites of ACC1 phosphorylation recognition motif (Shi, Chen et al. 2014, Kildegaard, Jensen et al. 2016). By mutating the putative phosphorylation sites from serine to alanine $\left(\mathrm{ACC} 1^{\mathrm{S} 659 \mathrm{~A}, \mathrm{~S} 1157 \mathrm{~A}}\right)$, the ACC 
activity increased by more than 3-fold and the production of two malonyl-CoA derived metabolites, FAEEs and 3-HPs were also increased.

\subsubsection{Fatty acids production through engineering malonyl-CoA synthetase} pathway

In addition to the major malonyl-CoA synthesis route from acetyl-CoA by ACC, malonyl-CoA can also be directly synthesized in S. cerevisiae. Overexpression of a plant malonyl-CoA synthetase gene (AAE13) in S. cerevisiae that ligates the malonic acid and CoA to generate malonyl-CoA have resulted in 1.6-fold increase in lipid accumulation (Wang, Chen et al. 2014). In addition, MatB gene from the bacteria Rhizobium leguminosarium encodes a malonyl-CoA synthetase, which directly catalyzes the formation of the malonyl-CoA from malonate and CoA (Kim 2002). However, sufficient supplement of intracellular malonate and CoA are the prerequisite for malonyl-CoA synthesis by malonyl-CoA synthetase. S. cerevisiae does not produce malonate naturally and is unable to uptake exogenously supplied malonate in the form of malonic acid. To deal with this problem, A dicarboxylic acid plasma membrane transporter MAE1 (Schizosaccharomyces pombe) that could uptake exogenous malonic acid was successfully introduced into $S$. cerevisiae for the accumulation of cytosolic malonate (Chen and Tan 2013). 


\section{Chapter 3. Improving Saccharomyces cerevisiae ethanol} production and tolerance via RNA Polymerase II subunit Rpb7

\subsection{Introduction}

Inbreeding of strains with robustness under industrial conditions and high production capacities of desired-compounds, one major challenge is that cellular phenotypes are often regulated by hundreds of genes, which makes it difficult for conventional engineering methods to achieve desirable expression profile simultaneously. To address the complexity of eliciting optimal expression profile for desired phenotype, engineering strategies call for spontaneous modulation of global gene expression and metabolism shifts (Lin, Zhang et al. 2013). In recent years, engineering components of global transcription machinery has been explored to fulfill the requirement of fine-tuning or reprogramming microbial cellular transcription profile. In prokaryotic microbes, a few key regulators have been successfully engineered to alter Escherichia coli (E. coli) and Zymomonasmobilis phenotypes, including sigma factor $\sigma^{70}$ (Alper and Stephanopoulos 2007, Tan, Dai et al. 2015), alpha subunit of RpoA (Klein-Marcuschamer, Santos et al. 2009), exogenous regulator IrrE (Chen, Wang et al. 2011), global regulator Hha \& H-NS (Hong, Wang et al. 2010, Hong, Hegde et al. 2012), cAMP receptor protein (CRP) (Zhang, Chong et al. 2012, Chong, Geng et al. 2014). In eukaryotic microbes, the transcriptional machinery is more complex, with a large set of general and specific transcription factors involved(Cramer 2004). Only TATA-binding protein (Spt15) (Alper, Moxley et al. 2006, Liu, Liu et al. 2011), TATA-binding protein associated factor Taf25 (Zhao, Li et al. 2014), and zinc 
finger-containing artificial transcription factors (Park, Lee et al. 2003) have been successfully engineered to alter $S$. cerevisiae phenotypes so far. All these approaches focused on engineering a specific transcription factor (TF) to alter DNA-binding specificity and thus change global gene expression.

Apart from TFs, RNA polymerase II (RNAP II) plays a central role in synthesizing all mRNAs. It is the core enzyme of yeast global transcription machinery, which not only interacts with DNA, transcript RNA and regulatory proteins during mRNA synthesis, but also is involved in mRNA post-initiation regulation (Koleske and Young 1994, Armache, Kettenberger et al. 2003, Sainsbury, Bernecky et al. 2015). The finetuning of the subunits of RNAP II may also have the potential to induce perturbations on global transcription. In this work, instead of engineering a specific TF from $S$. cerevisiae, we tried to target RNA polymerase II subunit Rpb7 to improve yeast ethanol tolerance and production. Among the twelve subunits of RNAP II, Rpb7 serves as an 'mRNA coordinator' (Harel-Sharvit, Eldad et al. 2010) at different stages of genes expression, including: i) interacting with processing factors of RNAP II transcription apparatus and nascent RNA transcripts (Woychik and Hampsey 2002, Jasiak, Hartmann et al. 2008); ii) participating in mRNA export and decay (Lotan, Goler-Baron et al. 2007) (Figure 3). The multifunction of Rpb7 suggests the possibility of mutating Rpb7 to elicit cellular transcription profile change and achieve desired phenotypes in yeast.

As for bioethanol industry, ethanol inhibition during the production stage is one of the major causes that lead to decreased ethanol production and stuck fermentation (Thatipamala, Rohani et al. 1992, Gibson, Lawrence et al. 2007, Zhao and Bai 2009). 
The presence of high concentration ethanol may denature cellular protein, alter plasma membrane permeability and inhibit mitochondrial function, which could slow down glucose transport and eventually inhibit yeast growth and ethanol fermentation (Ansanay-Galeote, Blondin et al. 2001, Moon, Ryu et al. 2012). In industrial very high gravity (VHG) fermentation with media containing more than $250 \mathrm{~g} / \mathrm{L}$ sugar, yeast strain should survive under the $>10 \%(\mathrm{v} / \mathrm{v})$ ethanol and the ideal yield should be high $(>15 \%(v / v))$ (Auesukaree 2017). Previous works have successfully demonstrated the possibility of enhancing yeast ethanol production by improving its ethanol tolerance primarily. For example, the introduction of TPS1 (6-phosphate-trehalose synthase) from Saccharomycopsis fibuliger in S. cerevisiae resulted in better survival in $18 \%(\mathrm{v} / \mathrm{v})$ ethanol and $\sim 15 \%$ increase of final ethanol concentration (Cao, Chi et al. 2014). Inhibiting ATH1 (acid trehalase) expression promoted yeast growth in $8 \%(\mathrm{v} / \mathrm{v})$ ethanol and $\sim 100 \%$ increase in ethanol productivity (Jung and Park 2005). An ethanol-tolerant mutant generated from UV-C mutagenesis displayed $\sim 18 \%$ more ethanol accumulation than the wild-type (Thammasittirong, Thirasaktana et al. 2013). Based on the presumption that improved ethanol resistance would lead to enhanced ethanol production in yeast, in this work, the ethanol tolerance trait was chosen as the primary engineering target to isolate efficient ethanol producing strain.

Our group has successfully improved $E$. coli ethanol tolerance by engineering its global regulator cAMP receptor protein (CRP) before (Chong, Huang2 et al. 2013). Here, random mutagenesis library of Rpb7 was constructed and subjected to screening under ethanol stress. The isolated variant with elevated ethanol tolerance had also 
shown much enhanced ethanol titers during very high gravity (VHG) laboratory fermentation as compared to the control. Fermentation was further investigated and genome-wide DNA microarray analysis was performed to reveal cellular transcription profile change. To the best of our knowledge, this study is the first to demonstrate the possibility of engineering eukaryotic RNAP to alter global transcription profile and hence improve strain phenotypes.

\subsection{Materials and Methods}

\subsubsection{Strains and media}

E. coli DH5 $\alpha$ (Invitrogen, San Diego, USA) was used for cloning and cultured at $37^{\circ} \mathrm{C}$ in Lysogeny broth (LB) medium (Bactotryptone $10 \mathrm{~g} / \mathrm{L}$, Yeast extract $5 \mathrm{~g} / \mathrm{L}$, SodiumChloride 10g/L). S. cerevisiae BY4742 and CEN.PK2-1C were purchased from EUROSCARF (Frankfurt, Germany) and cultured in YPAD media (20 g/L peptone and $10 \mathrm{~g} / \mathrm{L}$ yeast extract supplemented with $20 \mathrm{~g} / \mathrm{L}$ glucose) at $30^{\circ} \mathrm{C}$. Recombinant $E$. coli and yeast strains were screened in LB containing $100 \mu \mathrm{g} / \mathrm{mL}$ ampicillin and YPAD containing $200 \mu \mathrm{g} / \mathrm{mL} \mathrm{G} 418$ respectively.

\subsubsection{Plasmid and mutant library construction}

Gene RPB7 was amplified from BY4742 genome using primer 1\&2 (see Table 1), and inserted between BamHI and XhoI of plasmid pRS41K (Euroscarf, Frankfurt, Germany). Native promoter $R P B 7 p$ was amplified using primer $3 \& 4$ containing $S a c I$ and BamHI site respectively, and inserted into plasmid pRS41K. CYCT1 was cut from plasmid p416 MET25 (ATCC ${ }^{\circledR} 87324^{\mathrm{TM}}$ ) using XhoI and KpnI and ligated with plasmid pRS41K. The resulting plasmid harboring $R P B 7 p-R P B 7-C Y C T 1$ expression cassette 
was denoted as $\mathrm{p} 41 \mathrm{~K}-R P B 7$.

Random mutagenesis library of $R P B 7$ was generated by error-prone PCR according to Gene Morph $^{\circledR}$ II Random Mutagenesis Kit (Agilent Technologies, CA, USA). Specifically, 30 40 ng DNA template was applied for the amplification of $R P B 7$ with primer $5 \& 6$. PCR program was set as $5 \mathrm{~min}$ at $95{ }^{\circ} \mathrm{C}, 30$ cycles of $95^{\circ} \mathrm{C}$ for $40 \mathrm{~s}$, $55^{\circ} \mathrm{C}$ for $45 \mathrm{~s}$, and $72{ }^{\circ} \mathrm{C}$ for $1.5 \mathrm{~min}$, followed by $10 \mathrm{~min}$ at $72{ }^{\circ} \mathrm{C}$. The PCR products $(4 \mu \mathrm{g})$ and BamHI \& XhoI double digested p41K-RPB7 plasmid $(1 \mu \mathrm{g})$ were electroporated into BY4742 strain using an Eppendorf® multiporator (Hamburg, Germany) following Benatuil's protocol (Benatuil, Perez et al. 2010).

Table 1 Primers used in plasmid construction and error-prone PCR

\begin{tabular}{|l|l|}
\hline \multicolumn{1}{|c|}{ Primer } & \multicolumn{1}{|c|}{ Sequence } \\
\hline 1 & GCAGGCGGATCCCTCAATTTCTGAGAATGTT \\
\hline 2 & GCGCCGGAATTCCAAGTGAT TAAATAGCACC \\
\hline 3 & C GAGCTC CGGGGTTCTTCGGGCCAAATGAA \\
\hline 4 & C GGATCC TCTCAGAAATTGAGTTATTTATA \\
\hline 5 & CCGTTTCTCCTCCTACACCATTCT \\
\hline 6 & GGACCTAGACTTCAGGTTGTCTAA \\
\hline
\end{tabular}

\subsubsection{Mutant identification}

The mutant library $\left(\sim 10^{8}\right)$ was cultured in 200 mL YPAD medium supplemented with elevated ethanol concentration (from $8 \%$ to $12 \%(\mathrm{v} / \mathrm{v})$ ). After three to five successive subcultures, the enriched cell culture diluted by $10^{6}-10^{7}$ was spread onto YPAD plates. Individual colonies showed improved ethanol tolerance were randomly picked for miniprep (Zymoprep II kit (Zymo Research, Orange, CA, USA)) and sent for DNA sequencing. To eliminate the possibilities of introducing mutations from plasmid backbone and host strain during enrichment selection, mutant $R P B 7$ gene 
fragment was digested and re-inserted into a fresh plasmid p41K-RPB7 backbone and retransformed into fresh BY4742 background, generating mutant M1 used in this study. In addition, plasmid $\mathrm{p} 41 \mathrm{~K}-R P B 7$ from $\mathrm{M} 1$ and its native version were also transformed into strain CEN.PK2-1C. The recombinants with mutated and native version of $\mathrm{p} 41 \mathrm{~K}-$ $R P B 7$ were denoted as CEN-M1 and CEN-P41K-RPB7 respectively.

\subsubsection{Mutant growth under stress}

Overnight cell culture was inoculated into $5 \mathrm{~mL}$ fresh YPAD medium containing $0 \%, 8 \%, 10 \%$ ethanol $(\mathrm{v} / \mathrm{v})$ with an initial OD600 of 0.1 . Both the mutant and the control growth was recorded by monitoring their absorbance at $600 \mathrm{~nm}$ respectively. 5 mL YPAD medium supplemented with $3.5 \mathrm{mM} \mathrm{H}_{2} \mathrm{O}_{2}, 80 \mathrm{mM}$ acetic acid, or $1.5 \mathrm{M}$ $\mathrm{NaCl}$ was used to test mutant oxidative, acetic and osmotic tolerance respectively. Lignocellulose hydrolysate components, i.e. levulinic acid (196 mM), furfural (1.16 $\mathrm{g} / \mathrm{L}), \mathrm{HMF}(17.5 \mathrm{mM})$, ferulic acid $(1 \mathrm{mM})$, vanillin $(13.1 \mathrm{mM})$ and $p$-coumaric acid $(12 \mathrm{mM})$ were supplemented into $5 \mathrm{~mL}$ YPAD medium respectively to test mutant tolerance towards them.

\subsubsection{Ethanol fermentation}

Both mutant and the control were grown in $50 \mathrm{~mL}$ YPAD medium in $200 \mathrm{~mL}$ flasks to accumulate large amount of cells for high inoculum fermentation (initial OD 600: 15). During laboratory VHG fermentation, the culture YPAD media was about 2/3 (33 $\mathrm{mL} / 50 \mathrm{~mL}$ ) of the overall capped test tube volume to achieve micro-aerobic conditions. The initial glucose was $300 \mathrm{~g} / \mathrm{L}$. Cell samples were taken every $6 \mathrm{~h}$ for OD600 measurement and the supernatant from centrifugation was collected for metabolites 
analysis described below.

The fermentation process was investigated by altering host strain background, initial glucose concentration (50 300 g/L) and initial $\mathrm{pH}(5 \sim 8)$.

\subsubsection{Analytical method}

The concentration of yeast metabolites was quantified chromatographically by an Agilent 1100 HPLC system equipped with a Refractive Index Detector (RID). Ethanol, glucose, acetic acid, and glycerol were separated using an Aminex HPX-87H Ion Exclusion Column (Bio-Rad, Hercules, USA) at $35^{\circ} \mathrm{C}$, with $5 \mathrm{mM}$ sulfuric acid mobile phase at a flow rate of $0.6 \mathrm{~mL} / \mathrm{min}$. All samples with two biological replicates were filtered through a $0.20-\mu \mathrm{m}$ filter before HPLC analysis.

\subsubsection{DNA Microarray and quantitative Real-Time Reverse Transcription PCR (qRT-PCR)}

Total cellular RNAs were extracted from both the mutant and the control using RNeasy® Mini Kit and RNase-Free DNase Set (Qiagen, Hilden, Germany) under the following two conditions: i) when cells reached early exponential phase (OD600 1.0) in YPAD; ii) after $12 \mathrm{~h}$ VHG fermentation. RNA quality and integrity was verified by gel electrophoresis, as well as by measuring 260/230 ratios with a Nano Drop 1000 spectrophotometer (Thermo Scientific, MA, USA). Two biological replicates of each sample were sent to Genomax Technologies (Singapore) for DNA microarray assay using Yeast (V2) Gene Expression Microarray, $8 \times 15 \mathrm{~K}$ Microarrays (Agilent technologies, USA). The obtained data was analyzed by Agilent Genespring GX software and the $p$-values were calculated by unpaired Student $t$-test. 
qRT-PCR were performed using StepOne Plus ${ }^{\mathrm{TM}}$ Real-Time PCR System (Applied Biosystems, MA, USA). The isolated RNA described above was reverse transcribed to cDNA by iScript cDNA Synthesis Kit according to manufacturer's protocols (Bio-Rad, CA, USA) with 500 ng mRNA as template. All primers used for qRT-PCR are listed in Table 2. qRT-PCR was performed in $20 \mu \mathrm{L}$ reaction mixture, containing $10 \mu \mathrm{L}$ $2 \times \mathrm{SYBR}^{\mathrm{TM}}$ Green master mix (Life Technologies, MA, USA), $2 \mu \mathrm{L}$ primers $(5 \mu \mathrm{M}), 6$ $\mu \mathrm{L} \mathrm{H}_{2} \mathrm{O}$ and $2 \mu \mathrm{L}$ cDNA. Gene expression level changes were calculated by $2^{-\Delta \Delta \mathrm{Ct}}$ method, using 18s rRNA (RDN18) as reference gene.

Table 2 Primers used in qRT-PCR

\begin{tabular}{|c|l|}
\hline Primer & \multicolumn{1}{|c|}{ Sequence } \\
\hline URA3-F & GTGGTGGGCCCAGGTATTGTTA \\
\hline URA3-R & CACCGGGTGTCATAATCAACCA \\
\hline URA1-F & CCATGGGTTTACCAAACGAAGG \\
\hline URA1-R & ACTTGTGGTTTCCCAGGCACAT \\
\hline URA2-F & TGGGAGGTTTAGGTTCCGGTTT \\
\hline URA2-R & AGATGGGGCTACAACGATGGAA \\
\hline RND18-F & TTAATGACCCACTCGGCACCTT \\
\hline$R N D 18-\mathrm{R}$ & GGTGAGTTTCCCCGTGTTGAGT \\
\hline FBA1-F & GCCGCTATGGACCAATGGTTAG \\
\hline FBA1-R & GCGATGTCACCAGCGTACAAAC \\
\hline MDH2-F & CGGTGGTGATGAAGTGGTCAAG \\
\hline MDH2-R & TGGGGAAGTTGTTCGCATCTTT \\
\hline TDH3-F & CAGCTAACTTGCCATGGGGTTC \\
\hline TDH3-R & ATGGAGCCAAACAGTTGGTGGT \\
\hline ITR1-F & TAAACCCGTGAACGACGAGGAT \\
\hline ITR1-R & GGTAGCCGCAGTGACAATTTCC \\
\hline$E N O 2-\mathrm{F}$ & CCTTCGCTGAAGCCATGAGAAT \\
\hline$E N O 2-\mathrm{R}$ & TCGTGACCAGCAGCCTTGATAG \\
\hline$P D C 1-\mathrm{F}$ & AAGGAAGCCGTTGAATCTGCTG \\
\hline$P D C 1-\mathrm{R}$ & TGGGACAGCAACTGGCTTGTAA \\
\hline$P D C 5-\mathrm{F}$ & CGCTCCAGCTGAAATTGACAGA \\
\hline
\end{tabular}




\begin{tabular}{|c|c|}
\hline PDC5-R & GAAGCACAAGCATCAGCCAAGA \\
\hline$E R G 20-\mathrm{F}$ & GGTTGGTGCATTGAGTTGTTGC \\
\hline$E R G 20-\mathrm{R}$ & GGTTTGGAAGGTGACCTCATGG \\
\hline$F E N 1-\mathrm{F}$ & TGTTCGAGCGTTATCCCCAAC \\
\hline$F E N 1-\mathrm{R}$ & AGGGGGCAAAGTGCTTAATGG \\
\hline
\end{tabular}

\subsubsection{Intracellular reactive oxygen species (ROS) level}

The intracellular ROS level of mutant and the control was measured using a sensitive probe 2', 7'-dichlorodihydrofluoroscein diacetate $\left(\mathrm{H}_{2} \mathrm{DCFDA}\right)$. Overnight culture was inoculated into fresh $5 \mathrm{~mL}$ YPAD medium until OD600 reached 1.0. Cells were washed twice with $10 \mathrm{mM}$ pH 7.0 potassium phosphate buffer (PPB), resuspended in $5 \mathrm{ml} \mathrm{PPB}$ supplemented with $10 \mu \mathrm{M} \mathrm{H}_{2}$ DCFDA, and incubated at $30^{\circ} \mathrm{C}$, $200 \mathrm{rpm}$ for $30 \mathrm{~min}$ in darkness. Cells were then lysed by vortex with glass beads (425$600 \mu \mathrm{m})$. Cell lysate was applied per well in a 96-well microplate (black background) to measure its fluorescence intensity by a Tecan Infinite 200 microplate reader (Mannedorf, Switzerland) with excitation at $485 \mathrm{~nm}$ and emission at $535 \mathrm{~nm}$. The relative fluorescence unit was normalized according to the total protein concentration in cell lysate, measured with Bradford's reagent in an Eppendorf ${ }^{\circledR}$ Biophomoter (Hamburg, Germany).

\subsubsection{PDC activity assay}

Cell extract was prepared by ultrasonication with $0.6 \mathrm{~mm}$ diameter glass beads at $0^{\circ} \mathrm{C}$ for 2 min with an ultrasonic processor (UP200S, Hielscher, Germany) following Flikweert's approach (Flikweert, L et al. 1996). Pyruvate decarboxylase activity was assayed at $25{ }^{\circ} \mathrm{C}$ by monitoring the rate of the pyruvic-acid-dependent oxidation of 
NADH (340 nm), with ADH as a coupling enzyme using a Shimadzu UV1800 spectrometer (Shimadzu Inc., Kyoto, Japan). The assay mixture (1 mL) consisted of: $40 \mathrm{mM}$ pH 6.5 imidazole-HCI buffer, $0.15 \mathrm{mM} \mathrm{NADH}, 0.2 \mathrm{mM}$-thiamine pyrophosphate, alcohol dehydrogenase $88 \mathrm{U} \mathrm{mL}^{-1}$ (Sigma Aldrich, St. Louis, MO, USA), $5 \mathrm{mM} \mathrm{MgCl}$, and cell free extract. The reaction was initiated by the addition of $50 \mathrm{mM}$ pyruvate. Enzyme activity was normalized by total protein concentration measured by Braford assay.

\subsection{Results}

\subsubsection{Mutant isolation}

Random mutagenesis library bearing $\sim 10^{8}$ clones was subjected to enrichment selection and $\sim 30$ individual colonies were randomly picked, sequenced, and their growth performance was tested under ethanol stress. The best mutant M1 was found to have two amino acid mutations, Y25N and A76T. To eliminate the effects of possible mutation from chromosome or plasmid backbone, M1 RPB7 gene fragment was reinserted into digested p41K-RPB7 backbone and retransformed into fresh BY4742 background. The newly transformed strain M1 was used in this study.

In the absence of ethanol stress, M1 displayed slightly better growth than BY-P41K (BY4742 strain harboring plasmid p41k) and BY-P41K-RPB7 (BY4742 strain harboring plasmid p41K-RPB7) (Figure 5A). With $8 \%(\mathrm{v} / \mathrm{v})$ ethanol present (Figure 5B), the growth rate of M1 was $0.427 \mathrm{~h}^{-1}$, doubling that of the BY-41K and BY-P41KRPB7. When ethanol concentration was further increased to 10\% (v/v) (Figure 5C), a sub-lethal ethanol environment, M1 displayed a modest growth rate at $0.021 \mathrm{~h}^{-1}$, with a 
prolonged exponential phase, whereas the growth of BY-P41K-RPB7 was completely inhibited. When the native $R P B 7$ promoter of M1 was replaced by constitutive promoter TEF1p, the resulting variant showed no further improvement in $8 \%(\mathrm{v} / \mathrm{v})$ ethanol as compared with M1, which implied that overexpression of mutant RPB7 might not lead to better ethanol resistance (data not shown). The ethanol resistance of M1 was further exploited through ethanol susceptibility assay on agar plates. As shown in Figure 5D, M1 exhibited better survival than BY-P41K and BY-P41K-RPB7 when exposed to $10 \%$ (v/v) ethanol. As BY-P41K and BY-P41K-RPB7 displayed similar growth in ethanol, BY-P41K-RPB7 was denoted as the control in the following experiments. Since yeast cell with $R P B 7$ null mutation is inviable, the control here bore native $R P B 7$ gene to neutralize the influence and interference caused by plasmid and chromosomal copies of $R P B 7$. 

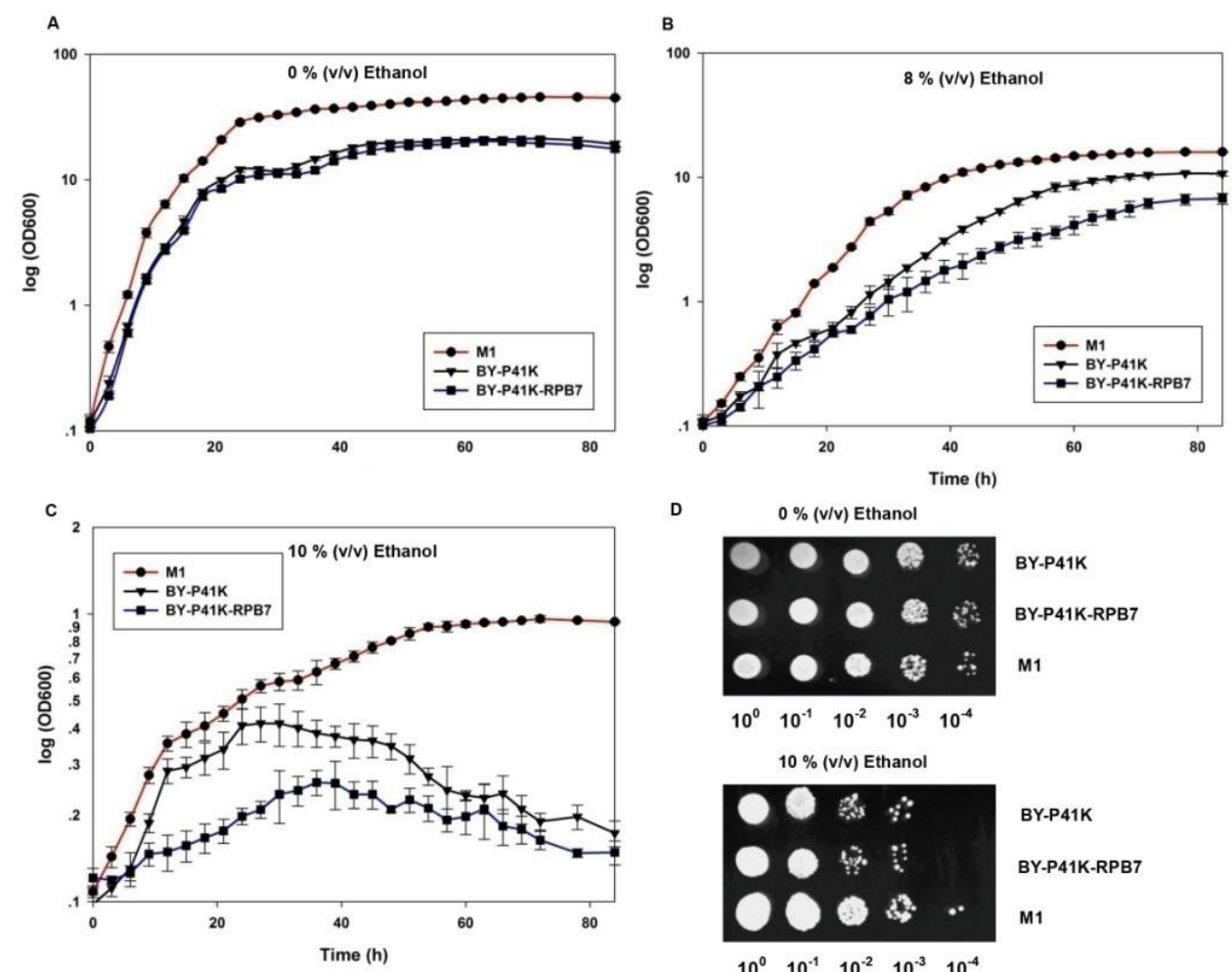

D

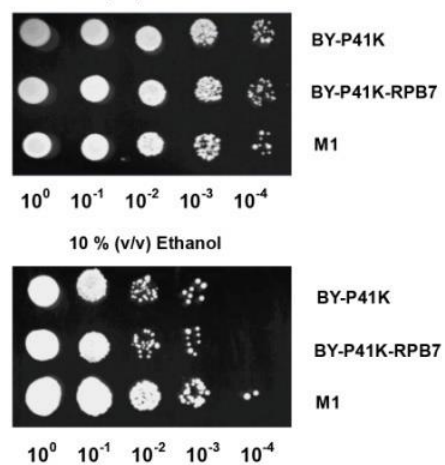

Figure 5. Ethanol tolerance. Ethanol tolerance of M1 in (A) 0\% ethanol; (B) 8\% (v/v) ethanol; (C)

$10 \%(v / v)$ ethanol. All cells were grown in YPAD medium at $30^{\circ} \mathrm{C}, 225 \mathrm{rpm}$. (D) Spot assay. Ten-fold serial dilutions of the culture $(5 \mu \mathrm{L})$ were spotted on YPAD agar with or without $10 \%(v / v)$ ethanol. The spotted agar plates were incubated at $30^{\circ} \mathrm{C}$ for 2 days. 


\subsubsection{Cross tolerance of M1}

Previous studies have shown that intracellular ethanol may denature proteins and generate reactive oxygen species (ROS), which inhibit glycolysis, amino acid transport pathways and mitochondrial function, and impose oxidative damage to cellular lipids, proteins and DNA (Costa, Amorim et al. 1997, Du and Takagi 2007). $\mathrm{H}_{2} \mathrm{O}_{2}$ is a typical stressor when testing strain oxidative stress tolerance (Izawa, Inoue et al. 1996, Spencer, Phister et al. 2014), hence $\mathrm{M} 1$ was subjected to $3.5 \mathrm{mM} \mathrm{H}_{2} \mathrm{O}_{2}$. As expected, $\mathrm{M} 1$ showed better growth than the control (Figure 6A). In addition, we tested acetae and $\mathrm{NaCl}$ as stressors - acetate is the by-product from ethanol fermentation and $\mathrm{NaCl}$ could lower the maximum specific growth rate of S. cerevisiae during fermentation (Watson 1970, Bai, Anderson et al. 2008). As shown in Figure 6, M1 exhibited enhanced resistance towards $80 \mathrm{mM}$ acetic acid (Figure 6B) and $1.5 \mathrm{M} \mathrm{NaCl}$ (Figure 6C) respectively.
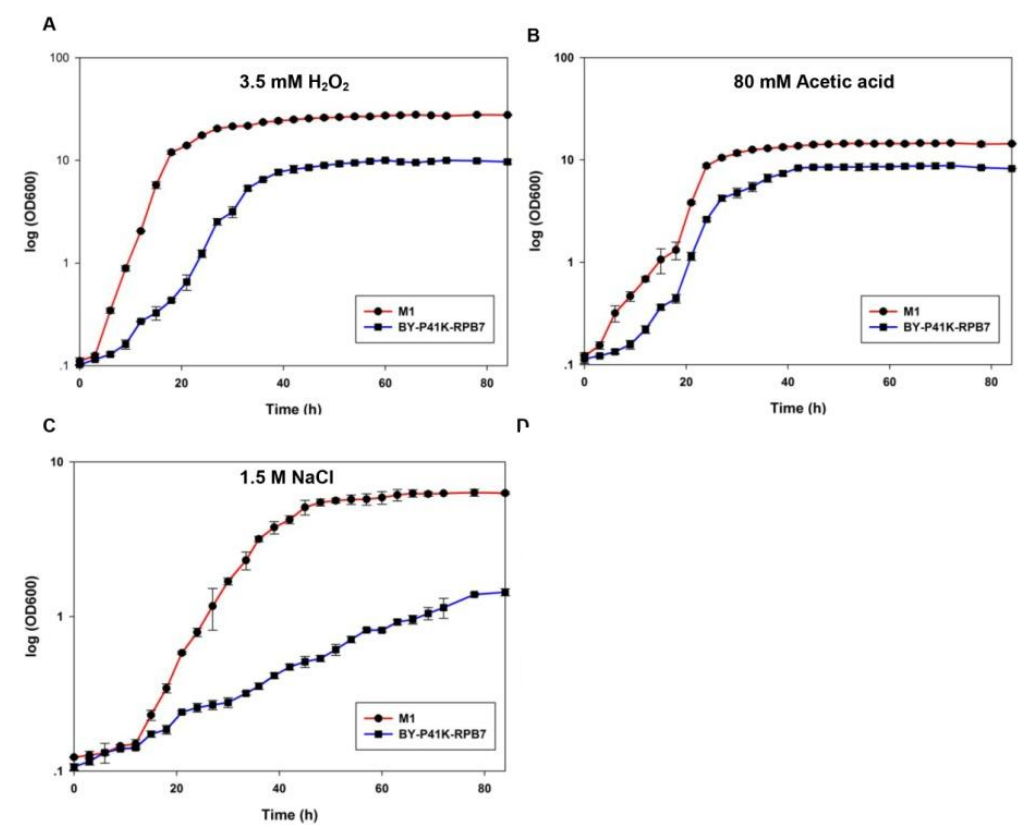
Figure 6. Cross tolerance towards stressors. The tolerance of $\mathrm{M} 1$ in (A) $3.5 \mathrm{mM} \mathrm{H}_{2} \mathrm{O}_{2}$; (B) $80 \mathrm{mM}$ acetic acid; (C) $1.5 \mathrm{M} \mathrm{NaCl}$.

Moreover, M1 also exhibited cross tolerance towards inhibitors from lignocellulose hydrolysates, including levulinic acid, furfural, HMF, and phenolic compounds (ferulic acid, vanillin, $p$-coumaric acid). When challenged by these inhibitors, M1 displayed better growth as compared to the control (Figure 7).
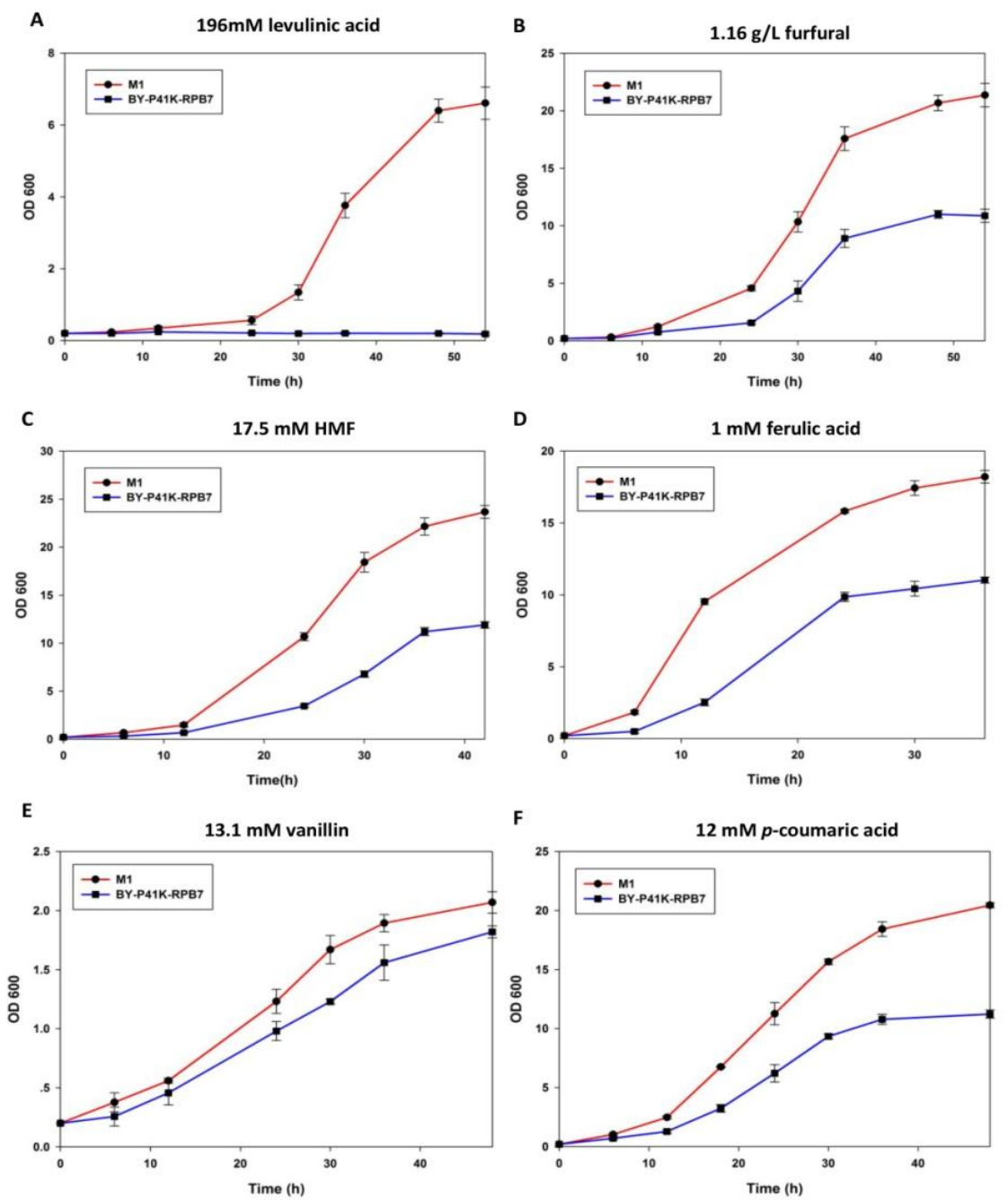

Figure 7. Cross tolerance towards inhibitors from lignocellulose hydrolysates.M1 in (A) $196 \mathrm{mM}$ levulinic acid; (B) $1.16 \mathrm{~g} / \mathrm{L}$ furfural; (C) $17.5 \mathrm{mM}$ HMF; (D) $1 \mathrm{mM}$ ferulic acid; (E) $13.1 \mathrm{mM}$ vanillin; (F) $12 \mathrm{mM} \mathrm{p-coumaric} \mathrm{acid.}$ 


\subsubsection{Intracellular ROS level}

As intracellular ROS level was an indicator of cell resistance towards toxic substances, it was also investigated in M1 (Demasi, Pereira et al. 2006). M1 seemed to have stronger capability in scavenging intracellular ROS as the intracellular ROS level of M1 was only $\sim 37 \%$ of that of the control when cells were collected at early log phase (Figure 8).

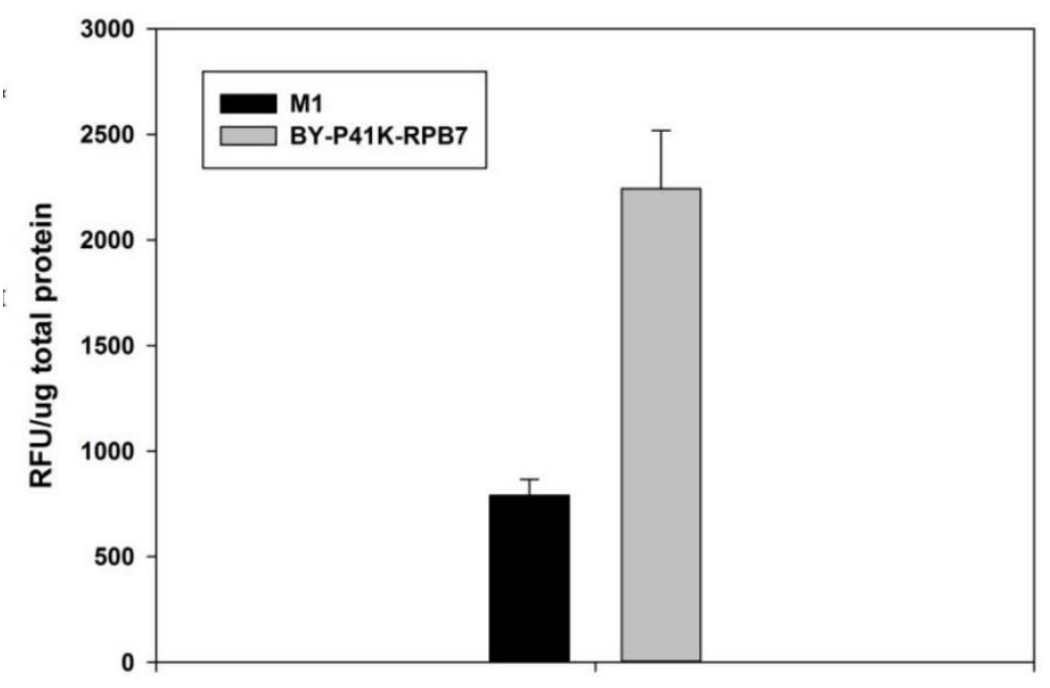

Figure 8. Intracellular ROS level in M1 when cells reached early log phase (OD600=1). ROS level is positively correlated to the fluorescence intensity of probe H2DCFDA. All experiments were performed in triplicates.

\subsubsection{Ethanol fermentation}

VHG laboratory fermentation with high-cell-density and high-glucose cultures under micro-aerobic condition was applied to M1, mimicking industrially relevant conditions (Bai, Chen et al. 2004). With an initial inoculum of OD600 $\sim 15(\sim 6 \mathrm{~g}$ DCW/L) and 300g/L glucose supply, the fermentation performance of M1 exceeded that of the control by showing better ethanol accumulation \& productivity (Figure 9A), 
rapid glucose consumption rate and improved biomass production (Figure 9B). Ethanol titers in M1 reached $122.85 \mathrm{~g} / \mathrm{L}$ after $54 \mathrm{~h}$, approximately $96.58 \%$ of the theoretical yield $(0.51 \mathrm{~g} / \mathrm{g}$ glucose $), \sim 40 \%$ increase as compared to that of the control $(87.75 \mathrm{~g} / \mathrm{L})$. M1 also displayed $\sim 33 \%$ increase in specific productivity $\left(0.541 \mathrm{~g} \cdot \mathrm{g} \mathrm{DCW} \mathrm{DC}^{-1} \mathrm{~h}^{-1}\right)$ over the control $\left(0.407 \mathrm{~g} \cdot \mathrm{g} \mathrm{DCW} \mathrm{DW}^{-1} \mathrm{~h}^{-1}\right)$ during the initial $12 \mathrm{~h}$ of fermentation. As shown in Table 3, M1 displayed higher cell density and ethanol productivity $(+39.99 \pm 1.84 \%)$ after $54 \mathrm{~h}$, surpassing that of the control. On the other hand, M1 showed similar level of byproduct acetic acid and glycerol as the control during fermentation (Figure 9C and 9D).

A

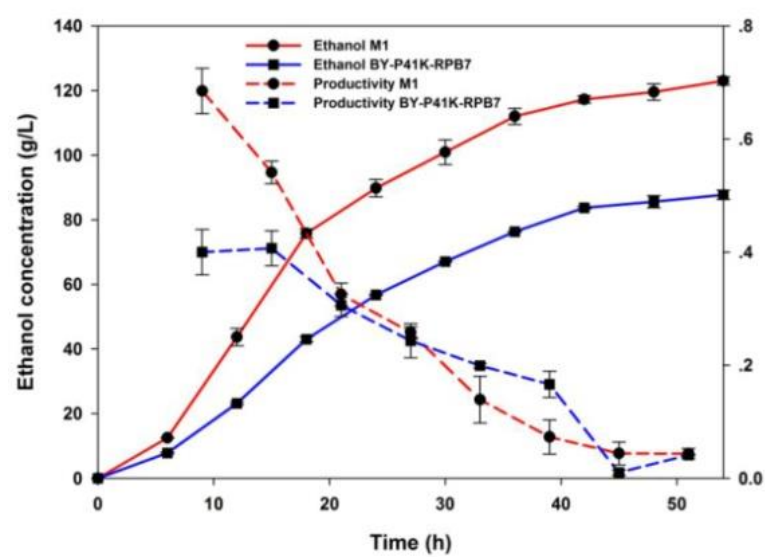

c

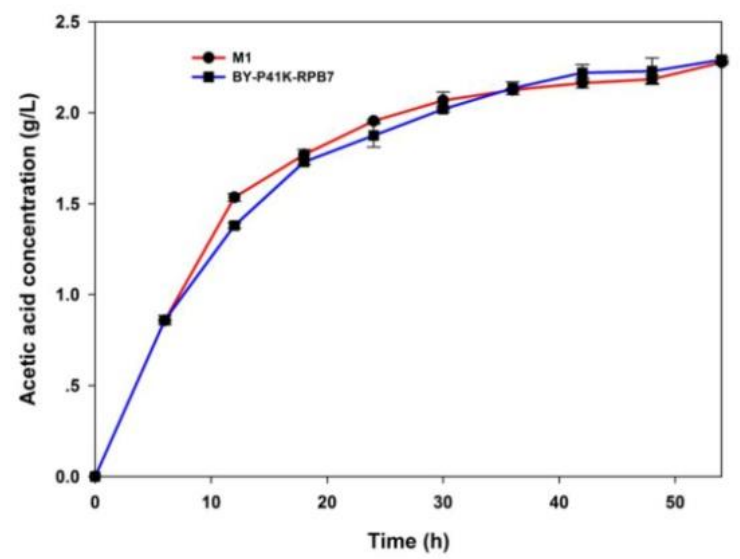

B

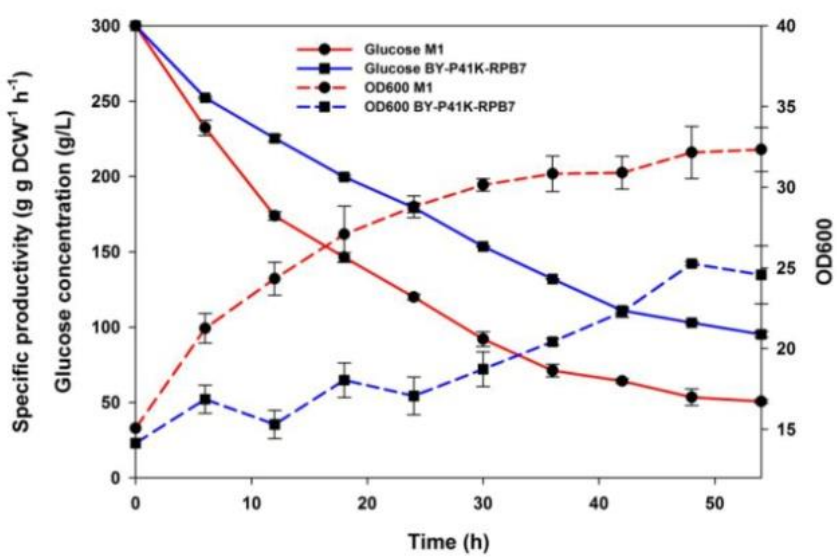

D

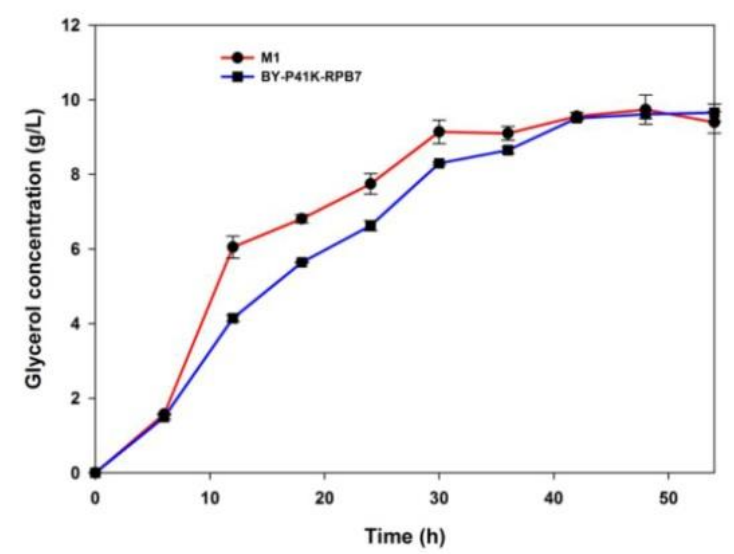


Figure 9. Fermentation characteristics during VHG laboratory fermentation. (A) ethanol concentration (solid line) and specific productivity (dashed line); (B) glucose concentration (solid line) and OD600 (dashed line); (C) acetic acid profile; (D) glycerol profile. Cells were cultured in biological replicates in $300 \mathrm{~g} / \mathrm{L}$ glucose with a high inoculum of initial cell density of OD600=15 ( $6 \mathrm{~g} \mathrm{DCW} / \mathrm{L})$. Metabolites were analyzed by HPLC.

*Specific productivity is expressed by ethanol productivity per viable cell following the equation below (Lam, Ghaderi et al. 2014):

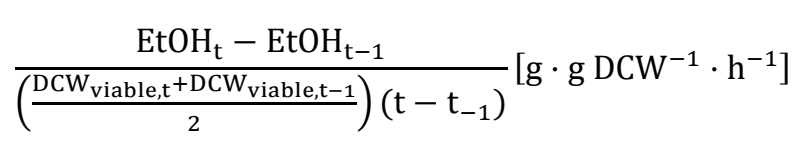

The rates of increase in ethanol titer over a specific period were normalized by the average viable cell density during the same period.

Table 3 Fermentation profiles of M1 and the control after $54 \mathrm{~h}$.

\begin{tabular}{cccc}
\hline Parameter & M1 & Control & $\begin{array}{c}\text { Percent } \\
\text { improvement }\end{array}$ \\
\hline \hline Initial DCW $(\mathrm{g} / \mathrm{L})$ & 6.02 & 5.66 & - \\
Final DCW $(\mathrm{g} / \mathrm{L})$ & $12.94 \pm 0.54$ & $9.83 \pm 1.08$ & $+65.94 \pm 16.08 \%$ \\
Ethanol titers $(\mathrm{g} / \mathrm{L})$ & $122.85 \pm 1.46$ & $87.75 \pm 1.30$ & $+39.99 \pm 1.84 \%$ \\
Ethanol yield $*$ & $96.58 \pm 1.14 \%$ & $83.99 \pm 1.24 \%$ & $+14.99 \pm 1.51 \%$ \\
& & & \\
\hline \hline
\end{tabular}

*Ethanol yield is expressed as percentage of the maximum theoretical ethanol yield $(0.51 \mathrm{~g}$ ethanol per gram of glucose consumed).

Fermentation was further investigated by varying initial glucose concentration, culturing $\mathrm{pH}$, and host strain background. M1 demonstrated improved glucose-ethanol conversion rate in the range of initial glucose concentration tested (Figure 10). On the other hand, the optimum initial $\mathrm{pH}$ was found to be 7 for VHG fermentation (Figure 
11). Therefore, we chose $\mathrm{pH} 7$ and initial glucose concentration at $300 \mathrm{~g} / \mathrm{L}$ as fermentation condition.
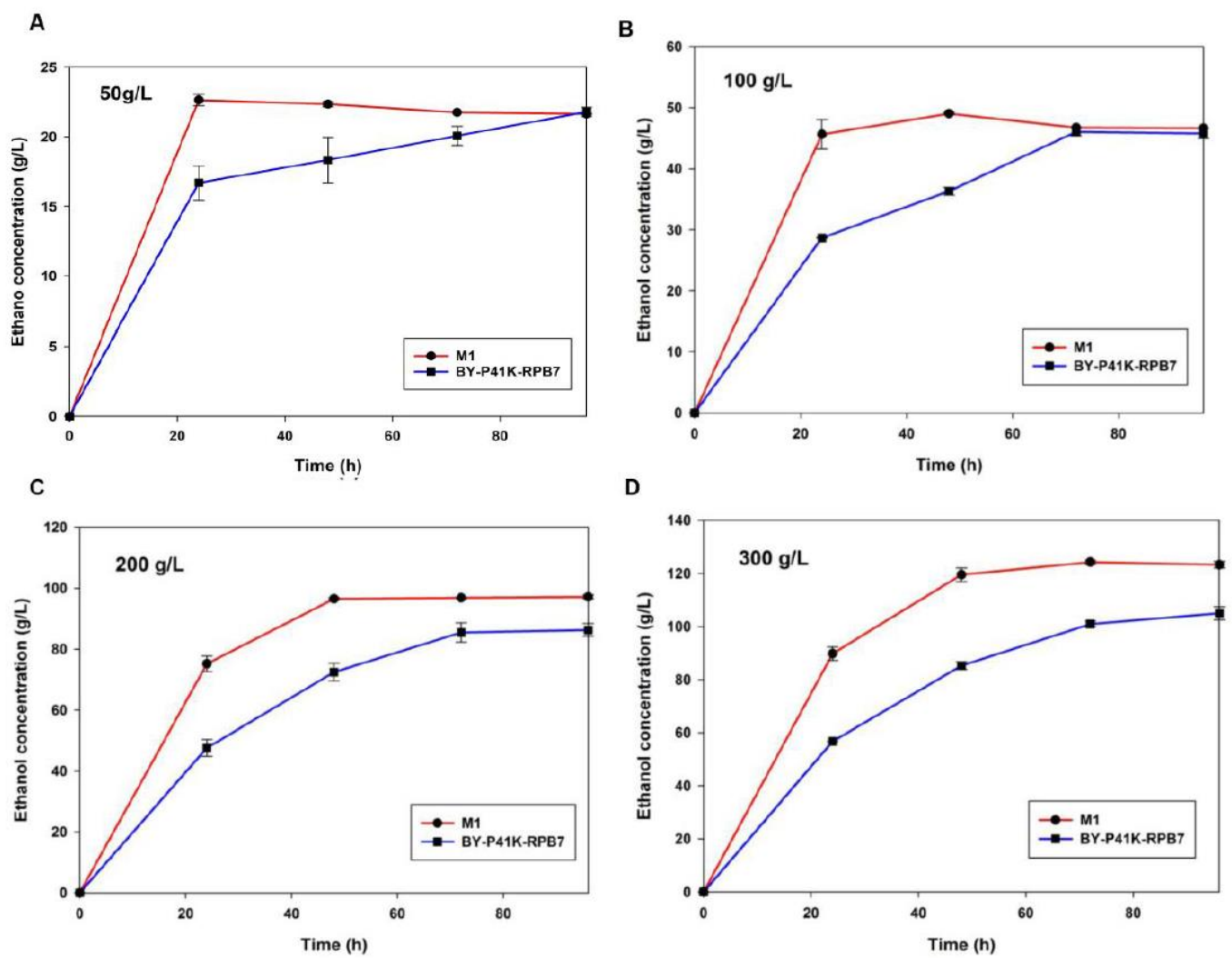

Figure 10. Ethanol profile with varying initial glucose supply under VHG fermentation (A) $50 \mathrm{~g} / \mathrm{L}$ glucose; (B) $100 \mathrm{~g} / \mathrm{L}$ glucose; (C) $200 \mathrm{~g} / \mathrm{L}$ glucose; (D) $300 \mathrm{~g} / \mathrm{L}$ glucose. 
A

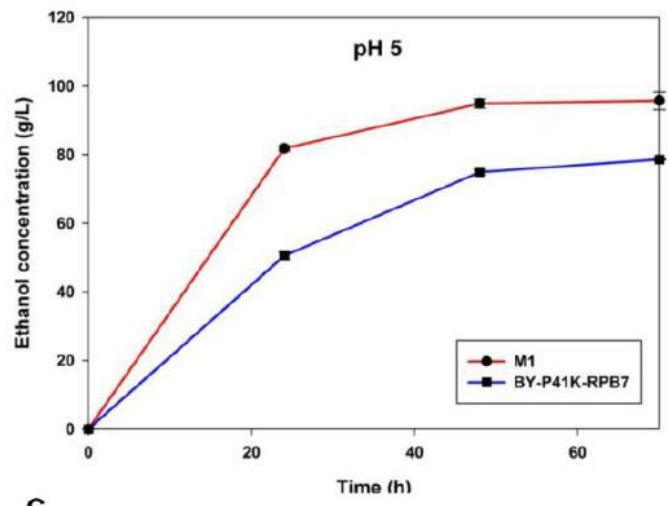

C

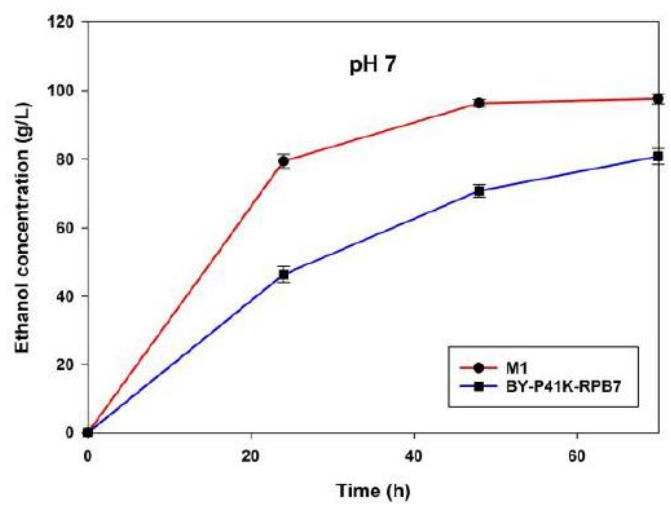

B

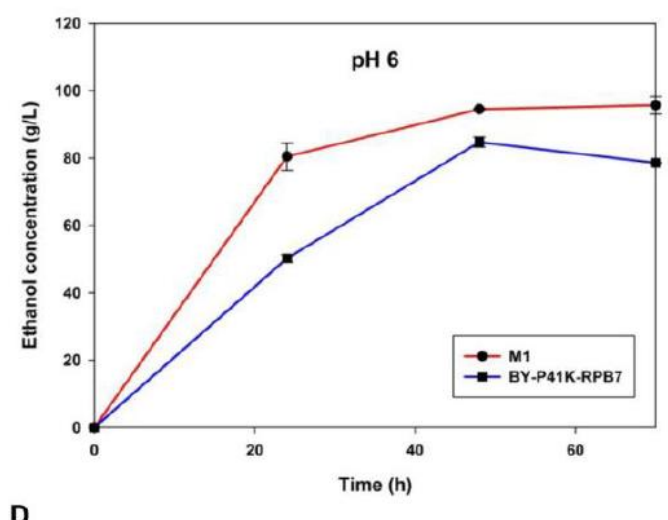

D

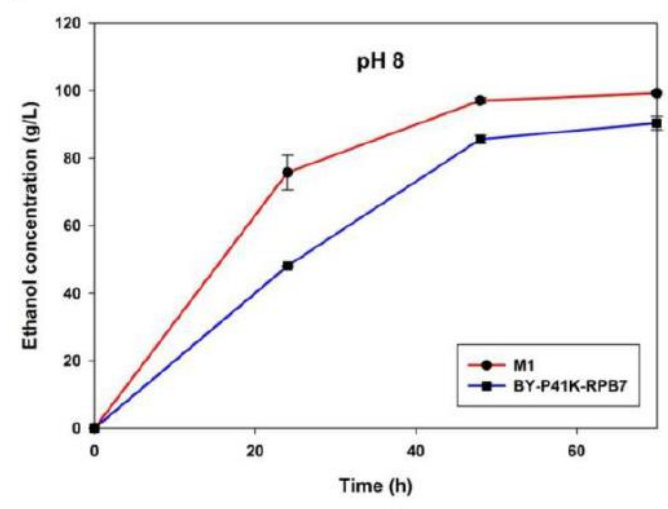

Figure 11. Ethanol profile with varying initial $\mathrm{pH}$ under VHG fermentation. (A) $\mathrm{pH} 5$; (B) $\mathrm{pH}$ 6; (C) pH 7; (D) pH 8. 
In order to study M1 ethanol fermentation characteristics in other yeast strain background, plasmid p41K-RPB7 from M1 and its native version were also transformed into strain CEN.PK2-1C, which property is more prone to industrial strains (Nijkamp, van den Broek et al. 2012). The recombinants with mutated and native version of $\mathrm{p} 41 \mathrm{~K}$ $R P B 7$ were denoted as CEN-M1 and CEN-P41K-RPB7 respectively. As shown in Figure 12, CEN-M1 and M1 exhibited similar fermentation properties. CEN-M1 also displayed higher ethanol accumulation $(\sim 18.2 \%)$ and faster glucose consumption $(\sim 12.5 \%)$ as compared to its control (CEN-P41K-RPB7) after $96 \mathrm{~h}$ of VHG fermentation.

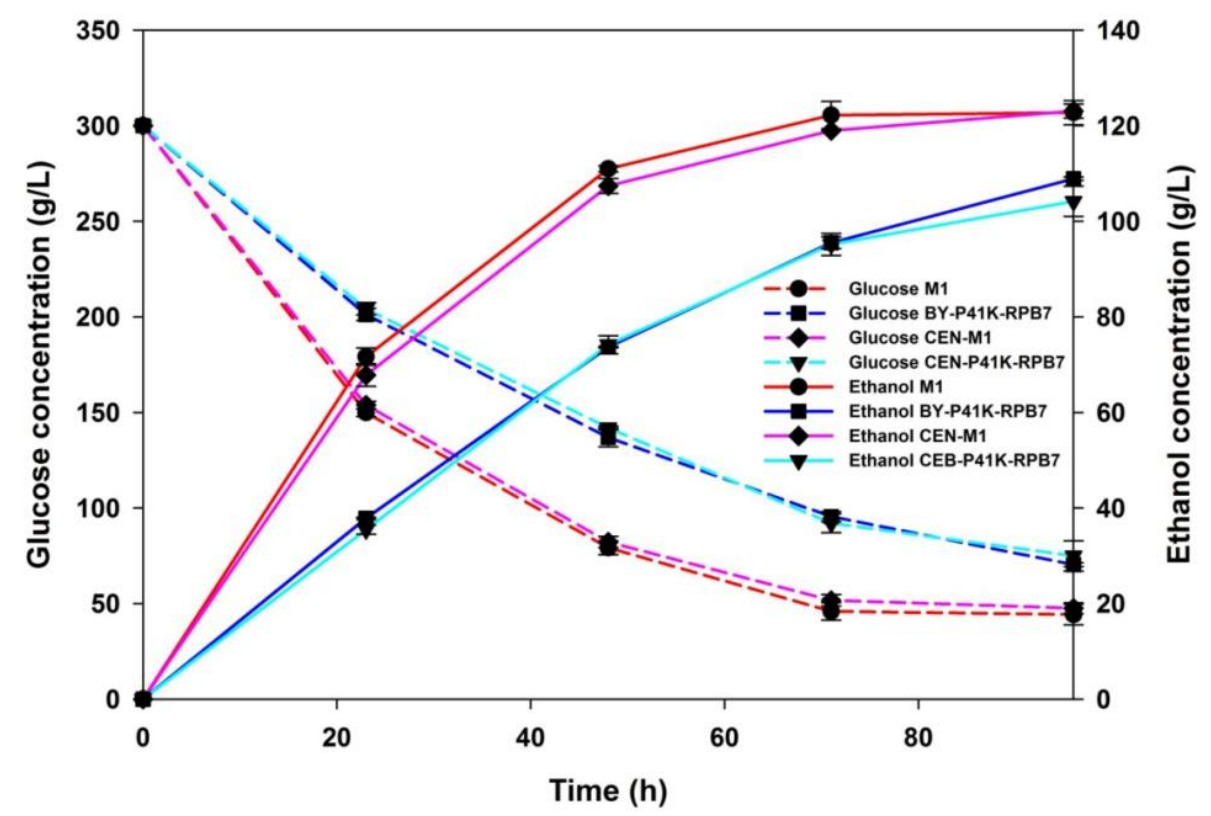

Figure 12 Fermentation characteristics in yeast strains. Ethanol production (solid line) and glucose consumption (dashed line) of M1 and the control in different yeast strains.CEN.PK2-1C strains containing mutated and native operon $\mathrm{p} 41 \mathrm{~K}-R P B 7$ were denoted as CEN-M1and CEN-P41K-RPB7 respectively. 


\subsubsection{DNA microarray assay and qRT-PCR results}

Genome-wide DNA microarray assay was carried out to quantify global transcription changes in M1. The mRNAs from M1 and the control were extracted after $12 \mathrm{~h} \mathrm{VHG}$ fermentation when M1 ethanol productivity was at its peak. Among the 6256 genes tested, 369 genes were observed with differential expression (>2-fold, $p$ value $<0.05)$ in M1, including 144 genes up-regulated and 225 genes down-regulated. All raw data are available from Gene Expression Omnibus (GEO, http://www.ncbi.nlm.nih.gov/geo/), with the accession number of GSE77853.

DNA microarray revealed that, among the 144 up-regulated genes, a significant set of gene are associated with energy metabolism, including glycolysis, alcoholic fermentation, hexose transport and $\mathrm{NAD}^{+}$synthesis. Specifically, among the ten enzymes involved in glycolysis, the following was found up-regulated, including hexokinase isoenzyme (HXK1, +2-fold), fructose 1,6-bisphosphate aldolase $(F B A, \quad+2.04$-fold $), \quad$ glyceraldehyde-3-phosphate dehydrogenase (TDH1/2/3, +2.10 2.21-fold), 3-phosphoglycerate kinase $(P G K 1, \quad+2.49$-fold $), \quad$ tetrameric phosphoglycerate mutase $(G P M 1,+2.02$-fold) and enolase $(E N O 1 / 2,+2 \sim 2.47$-fold $)$ (Figure 13). In the alcoholic fermentation pathway, increased induction of two pyruvate decarboxylase encoding genes ( $P D C 1$ and $P D C 5)$ was also observed. In addition, genes responsible for hexose transportation, HXT2/4/6/7 and de novo synthesis of NAD ${ }^{+}$, BNA1/4/5 were up-regulated by 2 4-fold in M1. 


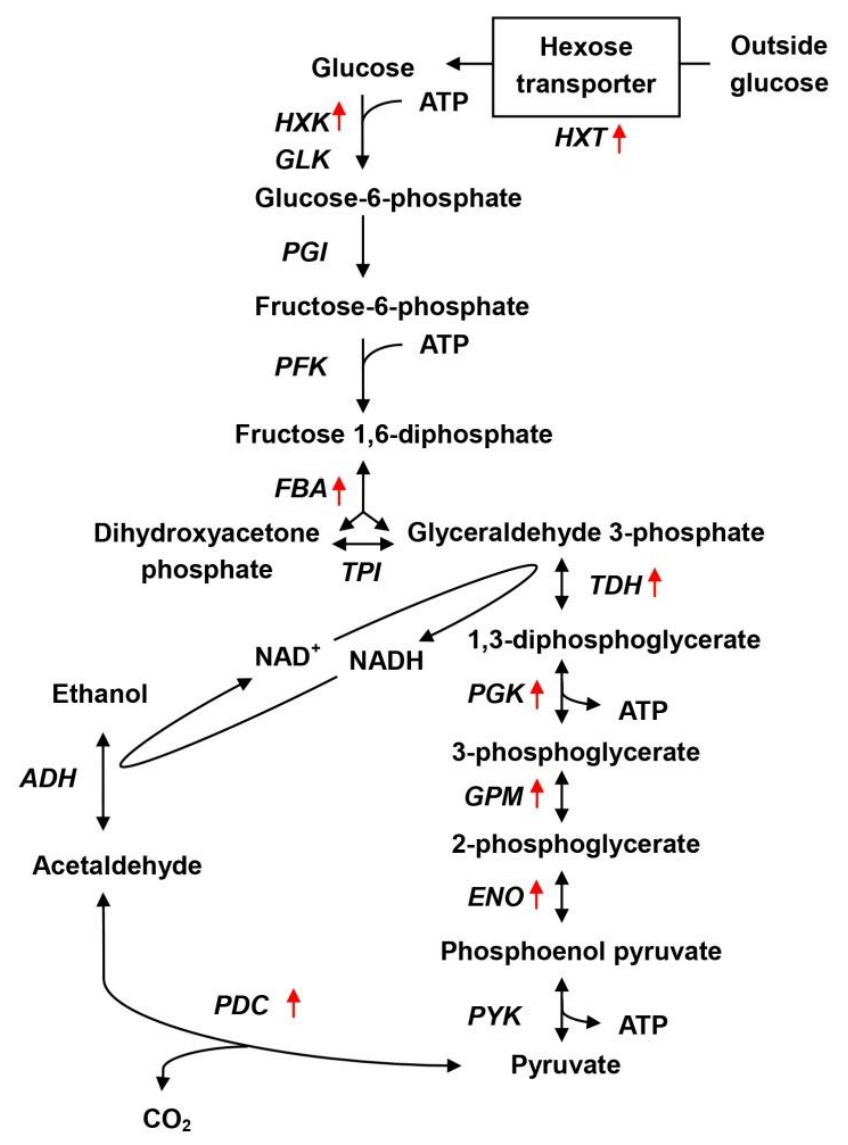

Figure 13. Ethanol synthesis pathway. Up-regulated genes (red arrows) from M1 in ethanol synthesis pathway.

Apart from genes involved in energy metabolism, the transcriptional reprogramming in M1 was quite broad, but it still demonstrated some enrichment of certain functional classes (Table 4). Oxidative stress response genes, such as TSA1 (+2.16-fold), TSA2 (+4.91-fold), SOD1 (+2.15-fold) displayed elevated expression. Genes involved in the long-chain fatty acids metabolism, including ACC1, FAS1, FEN1, OLE1 and SUR4, also showed up-regulation in M1 (+2.2 3-fold). Another group with enhanced expression is associated with sterol synthesis, namely ERG4, ERG20, HES1, UPC2 (+2 2.8-fold). While the major down-regulated genes ( $>2$-fold, $p$ value $<0.05)$ are from various functional groups, e.g. biosynthesis of pyrimidines (URA1, URA2, URA4), helicase activity (RRP3, YRF1-2/6/7,YIL177C), and DNA mismatch repair 
(MUS81, HOP2,MSH1). Those genes that are sensitive to nitrogen catabolite repression were also down-regulated in M1 (DAL3, DUR1,2, DUR3, DAL2).

Table 4 Differentially expressed genes in M1 after $12 \mathrm{~h}$ VHG fermentation

\begin{tabular}{|c|c|c|}
\hline Function group & Genes & $\begin{array}{l}\text { Log } 2 \text { fold } \\
\text { change* }\end{array}$ \\
\hline \multicolumn{3}{|l|}{ Up-regulated genes } \\
\hline Glucose, energy metabolism & $B N A 1$ & 4.08 \\
\hline \multirow[t]{2}{*}{ and ethanol pathway } & PDC5, & 3.63 \\
\hline & $\begin{array}{l}\text { PGK1, PDC1, HXT14, HXT6, HXT2, HXT7, BNA4, } \\
\text { ENO1, BNA5, TDH1, TDH2, TDH3, ENO2, ALD4, } \\
\text { FBA1, GPM1, HXK1 }\end{array}$ & $2-3.1$ \\
\hline \multirow[t]{2}{*}{ Oxidative stress } & TSA2 & 4.91 \\
\hline & $Y H B 1, T S A 1, S O D 1$ & $2-2.5$ \\
\hline Fatty acids synthesis & ACC1, FAS1, FEN1, OLE1, SUR4 & $2.2-3$ \\
\hline $\begin{array}{l}\text { Cell wall synthesis and } \\
\text { stability }\end{array}$ & $\begin{array}{l}\text { PIR1, YPS1, BAG7, GSC2, PSA1, CCW12, } \\
\text { YLR194C }\end{array}$ & $2.5-3.5$ \\
\hline Mental ion homeostasis & CTR1, SRO77, VMA3, IZH4, TIS11 & $2-3.1$ \\
\hline ER-associated trafficking & DFM1, GET3, CWH41 & $2-2.8$ \\
\hline Sterol synthesis & HES1, ERG4, ERG20, UPC2 & $2-2.8$ \\
\hline \multicolumn{3}{|l|}{ Down-regulated genes } \\
\hline \multirow[t]{2}{*}{ Biosynthesis of pyrimidines } & URA1, URA2 & $5.4-5.6$ \\
\hline & $U R A 4$ & 2.56 \\
\hline Transcription regulators & $\begin{array}{l}\text { GCN4, RRN7, SMP1, YAP5, SAS2, RBA50, RRN6, } \\
\text { IME1 }\end{array}$ & $2-2.7$ \\
\hline Helicase activity & $\begin{array}{l}\text { RRP3, YRF1-3, YRF1-6, YRF1-2, YRF1-7, YRF1-8, } \\
\text { YIL177C, YHR219W, YML133C }\end{array}$ & $2-2.5$ \\
\hline DNA repair & MSH1, HOP2, MUS81 & $2-2.3$ \\
\hline $\begin{array}{l}\text { Ribosome assembly and } \\
\text { function }\end{array}$ & RPS9B,RPF2, NSA3, YTM1, NOP6, NOP14, DRS1 & $2-3$ \\
\hline Sensitive to nitrogen & $D A L 3, D U R 1,2$ & $3.8-4$ \\
\hline catabolite repression & $D U R 3, D A L 2$ & $2-3$ \\
\hline
\end{tabular}

*All fold changes were significant, with $p$-value $<0.05$.

Twelve genes were selected for qRT-PCR to validate the consistency of microarray results (Table 5). 
Table 5 Comparison between DNA microarray and qRT-PCR results on selected genes from M1 after $12 \mathrm{~h}$ VHG fermentation.

\begin{tabular}{|l|l|l|l|l|}
\hline Gene Symbol & \multicolumn{2}{|l}{ DNA Microarray } & \multicolumn{2}{l|}{ qRT-PCR } \\
\hline & Log2 fold change & $\boldsymbol{p}$-value & Log2 fold change & $\boldsymbol{p}$-value \\
\hline URA3 & +13.15578449 & $6.93 \mathrm{E}-04$ & +10.479 & 0.0049 \\
\hline PDC5 & +3.629911 & 0.016687 & +1.826 & 0.000153988 \\
\hline ENO2 & +2.05134 & 0.001484 & +0.898 & 0.04774966 \\
\hline ERG20 & +1.010312659 & $5.78 \mathrm{E}-04$ & +3.976 & 0.03387 \\
\hline FEN1 & +1.163127384 & 0.006871 & +0.801 & 0.045055 \\
\hline ITR1 & +1.048042232 & $4.46 \mathrm{E}-05$ & +0.685 & 0.011993 \\
\hline PDC1 & +2.143487 & 0.047585 & +2.483 & $6.54026 \mathrm{E}-05$ \\
\hline TDH3 & +2.108383 & 0.009277 & +2.033 & 0.015969 \\
\hline MDH2 & -1.240953431 & 0.004 & -1.682 & 0.06595 \\
\hline$U R A 1$ & -2.439799814 & 0.001739 & -2.133 & 0.007919 \\
\hline$U R A 2$ & -2.467311386 & 0.001129741 & -3.02 & 0.00598 \\
\hline FBA1 & +2.045623 & 0.020591116 & +1.011 & 0.005983 \\
\hline
\end{tabular}

\subsection{Discussion}

Inspired by the advantage of gTME in bringing along high degree of pleiotropy efficiently (Klein-Marcuschamer, Santos et al. 2009), we directly engineered $S$. cerevisiae RNAP II subunit Rpb7 and successfully isolated an ethanol tolerant mutant via error-prone PCR. In accord with the assumption based on the correlation between cell viability and fermentation capability, M1 was also observed with improved ethanol titer of $122 \mathrm{~g} / \mathrm{L}$, which was comparable to the highest reported ethanol titer $(117.6 \mathrm{~g} / \mathrm{L})$ from its parental strain S288c in complex media with 33\% (w/v) glucose supply (Pais, Foulquie-Moreno et al. 2013). To the best of our knowledge, the highest reported ethanol, $\mathrm{H}_{2} \mathrm{O}_{2}, \mathrm{NaCl}$, acetic acid concentration yeast could tolerate and grow are $12 \%$ (v/v) (Kyung-Mi Yang, Ji-Min Woo et al. 2013), 5 mM (Spencer et al. 2014), 2 M (Goston et al. 2016), $110 \mathrm{mM}$ (Mira et al. 2015) respectively. M1 tolerance towards 
these stressors is less than the highest reported engineered strains. However, M1 improved osmotolerance and resistance towards byproduct acetic acid are valuable traits for industrial ethanol fermentation (Bai, Anderson et al. 2008). In addition, M1 showed improved growth in the presence of several lignocellulose hydrolysate inhibitors, indicating its potential in second generation bioethanol fermentation.

The two amino acid mutations in M1, Y25N and A76T, located at the N-terminus of Rpb7, which could interact with the core of RNAP II and bind single-stranded RNA in transcription (Cramer 2000). A76T is located at a conserved $\beta$-strand of the ribonucleoprotein (RNP)-like domain of Rpb7, forming a highly conserved 'tip loop' to interact with CTD linker of subunit Rpb1 \& Rpb6 and nascent RNA (Armache, Kettenberger et al. 2003, Choder 2004, Sampath and Sadhale 2005). Mutation Y25N lies within the $\alpha$-helix K2 of RNP, which also has putative RNA binding activity (Meka, Werner et al. 2005).

According to DNA microarray results, four high-affinity hexose transporter encoding genes (HXT2, HXT6, HXT7, HXT14) were up-regulated in M1, which assist glucose transport across plasma membrane, the first and rate-limiting step of glucose metabolism (Özcan and Johnston 1999). In addition, some genes involved in glycolysis pathway were also elevated in M1. The up-regulation of these genes may lead to faster glucose consumption in M1. Regarding the alcoholic fermentation pathway, qRT-PCR results showed that the expression of $P D C 1$ and $P D C 5$, which encode pyruvate decarboxylase, the key enzyme in ethanol synthesis, were both elevated by two-fold. Enzyme assay had also demonstrated that the activity of PDC in M1 was $28.3 \%$ higher 
than that of the control (Figure 14), indicating glycolytic flux into ethanol formation (Pronk, Steensmays et al. 1996). The observed overexpression of genes (BNA1, BNA4, BNA5) involved in de novo synthesis of $\mathrm{NAD}^{+}$from tryptophan may support the supplement of $\mathrm{NAD}^{+} / \mathrm{NADH}$ pool for boosted glycolysis and alcoholic fermentation (Vemuri, Eiteman et al. 2007). Hence engineering Rpb7 might stimulate spontaneous metabolism adjustment in the complex metabolic network.

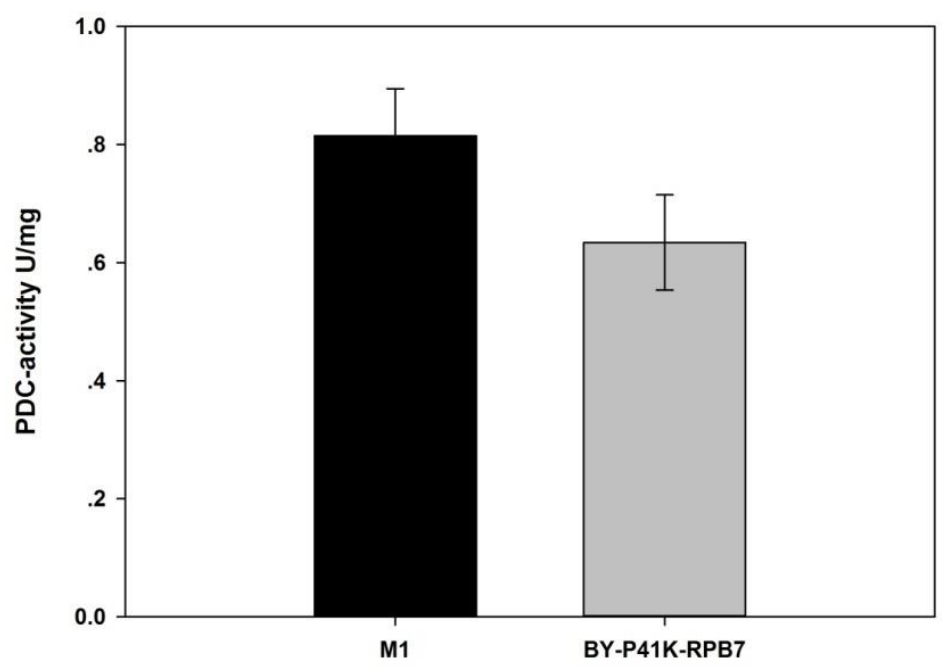

Figure 14 PDC activity in M1 and the control after 12h VHG fermentation.

The cross-tolerance conferred by M1 towards all these inhibitors suggested synergistic mechanism in response to fermentation challenges. DNA microarray results showed up-regulated expression in genes associated with cell oxidative stress response, including thioredoxin peroxidase TSA1 (+2.15-fold) and TSA2 (+4.9-fold), nitric oxide oxidoreductase YHB1 (+2.44-fold) and cytosolic copper-zinc superoxide dismutase SOD1 (+2.15-fold). In particular, thioredoxin peroxidase is a well-known cellular antioxidant (Wong, Zhou et al. 2002) that protects against cellular ROS toxicity in yeast. In addition, the transcription profile revealed that a set of up-regulated genes in M1 
were related to sterol and fatty acids metabolism, which were involved in ethanol stress defense. For example, it was demonstrated before that increased ergosterol content could improve ethanol tolerance by strengthening yeast membrane structure (Vanegas, Contreras et al. 2012). M1 also demonstrated elevated genes expression inergosterol synthesis pathway, i.e. ERG20 (farnesyl pyrophosphate synthetase), ERG4 (C-24(28) sterol reductase) and sterol synthesis regulation (HES1, UPC2). Previous work has shown that increased C18:1n-9 level could improve yeast ethanol tolerance (Kim, Kim et al. 2011) by reducing the fluidizing effects from ethanol (Henderson, LozadaContreras et al. 2013). Consistent with this finding, genes involved in the de novo biosynthesis of oleic (C18) acyl-CoA (C18:1n-9precursor), such as ACC1 (acetyl CoA Carboxylase I), FAS1 (fatty acid synthetase subunit), and FEN1 (fatty acid elongase) were also up-regulated in M1.

\subsection{Conclusion}

This work is the first to demonstrate that eukaryotic RNAP II enzyme could be an alternative gTME target in eliciting improved phenotype, which is probably also applicable to other eukaryotes. The increased ethanol titers in M1 and elevated expression of genes involved in ethanol production pathway indicates that it is possible to engineer RNAP II to change the expression of multiple genes simultaneously to enhance the yield of desired products. 


\section{Chapter 4. Systematic engineering for improving Saccharomyces cerevisiae alcohol tolerance}

\subsection{Introduction}

Although bioalcohol-producing industry experienced a negative influence by the oil price drop in 2014, biofuel is still considered as one potential substitute for traditional fuel (Rosillo-Calle 2006) since it is originated from renewable energy resource and the exhaust gases of bioalcohols (methanol, ethanol, propanol, butanol) burn more cleanly (Zhang, Rodriguez et al. 2011). S. cerevisiae has long been adopted as a superb ethanol producer and exploited as potential workhorse for higher alcohols. Nevertheless, for very high gravity (VHG) ethanol fermentation, which is common for industrial ethanol production, the accumulation of high concentration ethanol is a severe inhibitor for yeast viability and ethanol productivity (Puligundla, Smogrovicova et al. 2011). In addition, some evidences have shown the correlation between ethanol tolerance of cell proliferation and ethanol accumulating capacity (Lin and Tanaka 2006, Pais, FoulquieMoreno et al. 2013), i.e. better tolerance may lead to better production. In addition to producing ethanol, S. cerevisiae was also developed to produce butanol by introducing exogenous pathways (Steen, Chan et al. 2008) or utilizing endogenous pathway (Si, Luo et al. 2014). Its high sensitivities to butanol also pose the upper limits for cell growth and titer increase. To ameliorate the bioalcohol toxic effect, in-situ product removal processes (Ezeji, Qureshi et al. 2007) and bioreactor development are implemented (Liu, Wang et al. 2012), but alcohol-tolerant strain breeding is still essential for fermentation improvement. 
This review summarizes bioalcohol toxicity/tolerance mechanism (ethanol, propanol, butanol) in $S$. cerevisiae and engineering approaches developed for its tolerance improvement. The mechanisms reported so far are classified into genome and epigenome, transcription, translation, metabolism, and plasma membrane aspects. Counterpart engineering approaches are also introduced. In the case of unavailable genotype-phenotype information, classical strain engineering approach of adaptive evolution \& hybridization (Morales and Dujon 2012) and newly developed combinatorial strain engineering methods (Santos and Stephanopoulos 2008, Lin, Zhang et al. 2013), including, genome shuffling, random overexpression/knockout and global transcription machinery engineering are often employed (Figure 15).

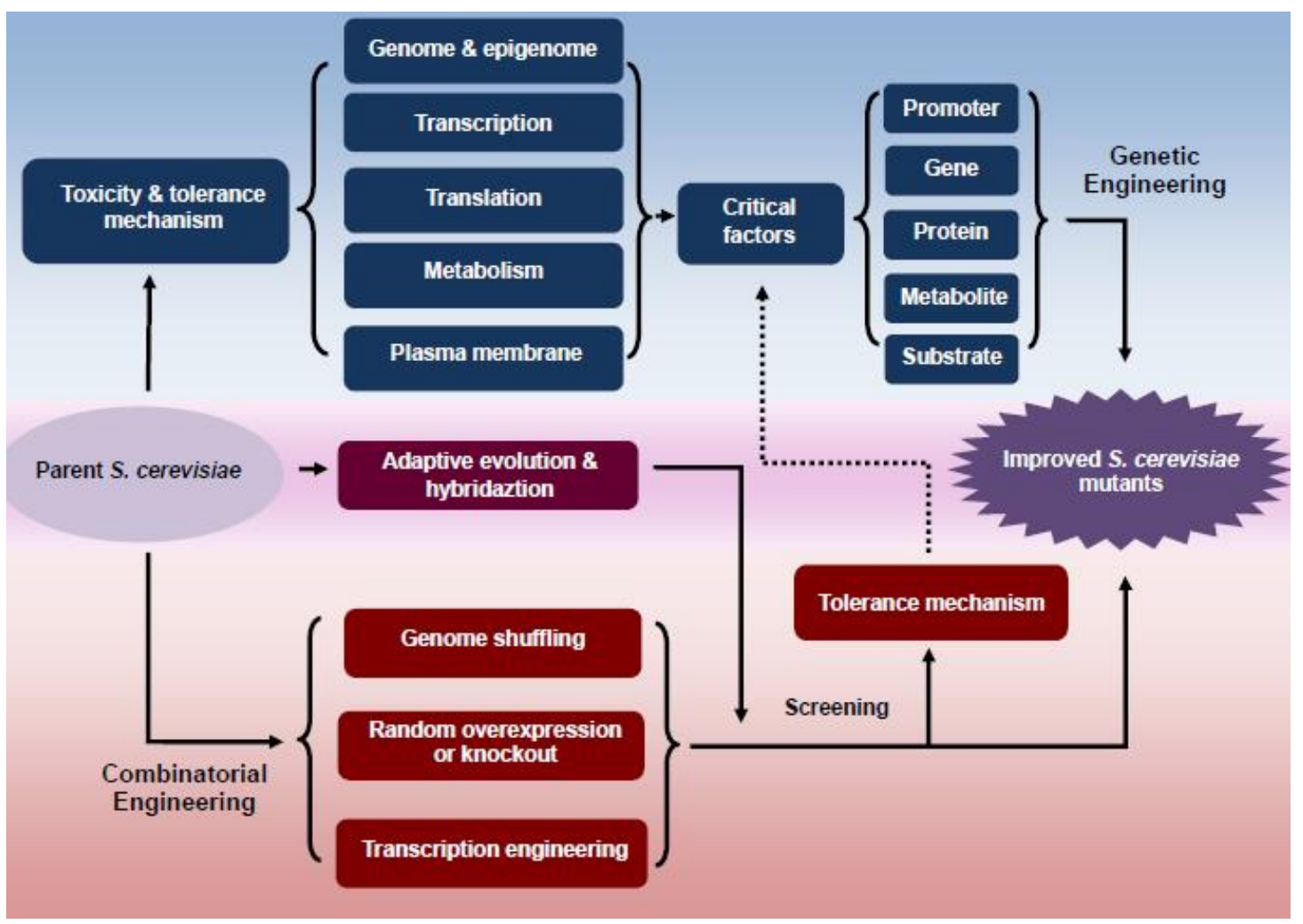

Figure 15. Strategic workflow of Saccharomyces cerevisiae engineering to improve its bioalcohol tolerance: i) mechanism-based strain engineering; ii) classical strain engineering of using adaptive evolution \& hybridization; iii) combinatorial engineering approaches. Mutants generated by these 
engineering approaches are screened under certain stressors. Tolerance mechanism generated from newly obtained mutation can also help identify new critical factors (dashed line).

\subsection{Bioalcohol toxicity/tolerance mechanism of $S$. cerevisiae and their respective engineering strategies}

Bialcohol toxicity/tolerance mechanism of $S$. cerevisiae has been reported from genetic and epigenetic, transcriptional, translational, metabolic, plasma membrane and other cellular aspects. The mechanism has inspired various strategies for strain engineering, i.e. rational engineering of bioalcohol relevant factors, including promoters, genes, proteins, metabolites, substrates and etc.

\subsubsection{From genome and epigenome aspect}

To uncover the molecular basis underlying $S$. cerevisiae bioalcohol toxicity/tolerance mechanism, genome-wide gene identification is a prerequisite. The candidates are generally originated from: studies on cell growth behavior of yeast knockouts under bioalcohol stress or analysis of global transcriptional variation caused by bioalcohol challenge. Single-gene knockout library was screened by comparing yeast knockout collection susceptibility to alcohol stress with parental strains. Over 600 genes from diverse gene ontology (GO) categories have been identified to be related with ethanol stress (Takahashi, Shimoi et al. 2001, Kubota, Takeo et al. 2004, Fujita, Matsuyama et al. 2006, Voorst, Houghton-Larsen et al. 2006, Teixeira, Raposo et al. 2009, Yoshikawa, Tanaka et al. 2009). The major overlap of the GO data belongs to three clusters, i.e. vacuolar $\mathrm{H}^{+}$-ATPase (V-ATPase), peroxisomal function and mitochondrion function. As ethanol pressure was demonstrated to induce increased 
intracellular and vacuolar acidity (Rosa and Sś-Correia 1996), to counteract this increase, V-ATPase would help on cytosolic $\mathrm{pH}$ recovery. Mutants deficient of VATPase were found co-sensitive to 1-propanol and 1-pentanol. Peroxisomal function is also required under ethanol stress, hypothetically due to its involvement in remodeling cell membrane (You, Rosenfield et al. 2003) and reactive oxygen species (ROS) metabolism (Schrader and Fahimi 2004). In addition, high proportions of mitochondrion-related genes were identified to be related with ethanol tolerance, including genes involved in respiration, biosynthesis and mitochondrial DNA maintenance, which suggests that respiration and carbohydrate metabolism is essential for cell ethanol stress resistance.

Quantitative Trait Loci (QTL) mapping, which allows genetic loci, gene polymorphism and polygenic trait analysis, can provide more complex genetic architecture compared with single gene identification. This approach starts by crossing parental strains with different bioalcohol tolerance levels and generates heterozygous $\left(F_{1}\right)$ segregant pool, which is subsequently crossed among itself or crossed to one of the parental strains to create $F_{2}$ individuals. Bioalcohol tolerance and the genotyping SNP markers of these $F_{2}$ individuals are scored. Markers linked to alcohol tolerance traits will present higher frequency in superior tolerant segregants whose loci and polymorphisms are analyzed (C.Mackay 2001). Regions bearing MKT1, SWS2 and APJ1 (Swinnen, Schaerlaekens et al. 2012), allele of VPS70 (Duitama, SánchezRodríguez et al. 2014) and chromosome 6, 7, 9, 12, 16 (Hu, Wang et al. 2007) were discovered by this method. Based on the QTL mapping data, Swinnen et al. further 
engineered BY4741 strain by knocking out APJl(encoding a chaperone during small ubiquitin-like modifier-mediated protein degradation) and overexpressing $M K T 1$ (encoding a protein mediating posttranscriptional regulation of heterothallic genes). Both mutants showed resistance towards $16 \%(\mathrm{v} / \mathrm{v})$ ethanol.

Yeast is known to utilize epigenetic control, for instance, chromatin remodeling, to cope with various stresses (Shivaswamy and Iyer 2008). Epigenetic regulation has been demonstrated as a dominant contributor to influence trans variability in yeast (Choi and Kim 2008), which has inspired novel engineering methods to improve yeast alcohol tolerance. For instance, histone deacetylases (HDACs) can control chromatin remodeling by regulating histone protein acetylation in S. cerevisiae (Grunstein 1997). The deletion of SIR3 and SIR4 (encoding silencing regulators in Class III HDAC function) exhibited enhanced ethanol tolerance (Matsuda, Fujimura et al. 2011). One possible reason could be that defective HDACs might loosen chromatin structure and stimulate the expression of stress defense-related genes. Another report revealed that lysine4 methylation of histone $\mathrm{H} 3(\mathrm{H} 3 \mathrm{~K} 4)$ could enhance ethanol tolerance as well (Wang, Ji et al. 2014), which was probably due to its influence on the transcription level of four ethanol-resistance-related genes (HSP104, PRO1, TPS1 and SOD1).

\subsubsection{From transcription aspect}

Another approach to reveal bioalcohol-relevant genes is through investigating global transcriptional changes in response to alcohol stress through DNA microarray and qRT-PCR. The transcriptome variation comes from either short-term $(<3 \mathrm{~h})$ or long-term (overnight) stress response. Several groups have indicated that short-term 
ethanol stimulus could induce the expression of hundreds of genes involved in heat shock response, oxidative response, energetic metabolism (trehalose, glycogen, glycerol metabolism and glycolysis), and vacuolar function (Alexandre, AnsanayGaleote et al. 2001, Lewis, Elkon et al. 2010, Ma and Liu 2010, Stanley, Chambers et al. 2010). Heat shock proteins (HSPs) have been reported to play an important role in preventing protein aggregation. Alexandre et al. observed a whole set of HSPs induced by ethanol shock, which supported the prediction that ethanol toxicity would affect protein folding. Genes involved in oxidative damage protection were found upregulated to avoid damaging effects from reactive oxygen species (ROS) caused by ethanol stress. The elevated expression of genes related to glycolysis, and thehalose, glycogen, glycerol metabolism and ATPase regulation are considered to meet the energy demand induced by short-term ethanol shock. In addition, vacuole's roles in $\mathrm{pH} /$ ion homeostasis and protein degradation maintenance are also important for strain ethanol tolerance. Yeast was found to exhibit high expression level of vacuolarfunction-related genes such as CPS1, LAP4, PEP4, VPS34, VPS36, SMI1, VPS16 and VPS28. On the other hand, the down-regulated genes after ethanol shock were mainly associated with protein synthesis, amino acid and nucleotide metabolism, which probably explains yeast growth arrest as a way to save energy (Chandler, Stanley et al. 2004).

Nevertheless, transcriptome under long-term alcohol treatment is very different from that of short-term stimulus. For example, Li et al. observed the up-regulation of genes responsible for ribosome biogenesis and lipid synthesis regulation after ethanol 
acclimation, which had few overlap with aforementioned genes (Li, Cheng et al. 2010). One possible explanation for this phenomenon, compared with the dynamic transcriptional response to ethanol stimulus, was that slight transcriptome alteration after long-term treatment could be a steady acclimation to new ethanol environment, as suggested by the authors.

Global transcription profile has inspired engineering approaches on trans-acting factors that can regulate bioalcohol-relevant genes. Shimoi group observed that $88 \%$ of the highly expressed genes under ethanol stress had stress response elements (STRE), i.e. promoter motif $\mathrm{CCCCT}$ and $\mathrm{AGGGG}$, which bind to transcription factor Msn4/Msn2 that activates stress-responsive transcription (Watanabe, Tamura et al. 2007). Overexpression of Msn2 resulted in enhanced gene expression with STRE promoters and improved microbe survival in $18 \%(\mathrm{v} / \mathrm{v})$ ethanol (Watanabe, Watanabe et al. 2009).

Ethanol also affect genes post-transcription. DNA microarray data can only provide insights at mRNA level in yeast, but cannot reflect mRNA post-transcription lifespan. It was reported that nucleocytoplasmic poly $(\mathrm{A})^{+}$RNA export was blocked by $10 \%(\mathrm{v} / \mathrm{v})$ ethanol, whereas heat-induced SSA4 and SSA1 mRNA could still be exported from the nucleus to cytoplasm under the same condition, which indicated selective mRNA transport in yeast (Saavedra, Tung et al. 1996, Takemura, Inoue et al. 2004). Stanley et al. concluded that this selective mRNA transport was specific for ethanol stress as compared to other stressors (Stanley, Bandara et al. 2010). The phenomenon was further studied by Izawa group, and it was suggested that the hyper-adenylation of HSP 
mRNAs (with poly(A) tails) could affect their export and ultimately control such HSPs expression level under ethanol stress (Izawa, Kita et al. 2008).

\subsubsection{From translation aspect}

Proteomic profile is useful to link genome and proteome to cell function as compensation for genomic and transcriptomic studies since mRNA abundance cannot represent gene expression quantitatively (Gygi, Rochon et al. 1999). Santucci group had used 2D electrophoresis technology to detect yeast translation alteration during ethanol accumulation (Trabalzini, Paffetti et al. 2003). Besides the aforementioned ethanol stress-associated proteins, it is worth noticing that there are other induced proteins under ethanol stress, which are involved in intracellular proteolysis, such as vacuole/proteasome autoproteolytic process including Bmh1, Bmh2 (14-3-3 proteins, conserved regulatory molecules controlling proteome at post-transcriptional level), Eno1 (enolase I, a phosphopyruvate hydratase), and Gpm1 (tetrameric phosphoglycerate mutase) that participates in proteasome interaction, Ubi4 (ubiquitin) and Tfs1 (natural metastasis suppressor) from protein degradation, and Sod1 (cytosolic copper-zinc superoxide dismutase) related with vacuole biogenesis. The enhanced proteins level that participated in proteolysis is considered as ethanol cellular defense in response to the aggregated unfolded proteins caused by alcohols. The induction of these proteolysis proteins could be considered as fine-tuning of recycling nitrogen source and balancing oxidation-reduction potential under critical ethanol stress (Mauricio, Valero et al. 2001). 


\subsubsection{From metabolism aspect}

Previous research has shown that the metabolism involved in trehalose, cofactor $\mathrm{NAD}^{+} / \mathrm{NADH}$ and proline balance is crucial for yeast bioalcohol tolerance. Trehalose can stabilize plasma membrane as well as assist intracellular protein folding when challenged with ethanol (Mansure, Panek et al. 1994, Singer and Lindquist 1998). Although the protective role of trehalose had been questioned previously (Alexandre, Plourde et al. 1998), a recent report indicated that it could only protect yeast in lethal ethanol concentration, i.e. $>10 \%(\mathrm{v} / \mathrm{v})$ (Bandara, Fraser et al. 2009). It was reported that chromosomal integration of TPS1 (encoding trehalose-6-phosphate synthase) from $S$. fibuligera A11 and heterologous expression of SALC (encoding trehalose-6-phosphate synthase) from Streptomyces albus could improve trehalose synthesis in $S$. cerevisiae respectively and render enhanced viability (Moon, Ryu et al. 2012, Cao, Chi et al. 2014) (Table 6). In addition, the deletion of either cytosolic neutral trehalase Nth1 or Nth2 and the inhibition of vacuolar acid trehalase Ath1 (Jung and Park 2005) could also deliver improved ethanol tolerance along with better fermentation performance.

Table 6. Examples of metabolism-based engineering approaches to improve $S$. cerevisiae bioalcohol tolerance

\begin{tabular}{|c|c|c|c|c|c|c|}
\hline Bioalcohol & Target & $\begin{array}{c}\text { Target } \\
\text { Description }\end{array}$ & $\begin{array}{c}\text { Genetic } \\
\text { Manipulation }\end{array}$ & $\begin{array}{c}\text { Phenotype } \\
\text { improvement } \\
\text { in alcohol \% } \\
(\mathrm{v} / \mathrm{v})\end{array}$ & $\begin{array}{l}\text { Production } \\
\text { Improvement }\end{array}$ & Reference \\
\hline Ethanol & Tps 1 & $\begin{array}{l}\text { Trehalose-6- } \\
\text { phosphate } \\
\text { synthase from } \\
\text { S. fibuligera }\end{array}$ & $\begin{array}{c}\text { Heterologous } \\
\text { Expression }\end{array}$ & $\begin{array}{l}\text { Survival in } \\
18 \% \text { ethanol }\end{array}$ & $\sim 15 \%$ & $\begin{array}{l}\text { (Cao, Chi } \\
\text { et al. } \\
\text { 2014) }\end{array}$ \\
\hline
\end{tabular}




\begin{tabular}{|c|c|c|c|c|c|}
\hline $\mathrm{SalC}$ & $\begin{array}{l}\text { Trehalose-6- } \\
\text { phosphate } \\
\text { synthase from } \\
\text { Streptomyces } \\
\text { albus }\end{array}$ & $\begin{array}{c}\text { Heterologous } \\
\text { expression }\end{array}$ & $\begin{array}{l}\text { Survival in } \\
10 \% \text { ethanol }\end{array}$ & $13 \%$ & $\begin{array}{l}\text { (Moon, } \\
\text { Ryu et al. } \\
\text { 2012) }\end{array}$ \\
\hline Nth1 & $\begin{array}{c}\text { Neutral } \\
\text { trehalase }\end{array}$ & Knockout & $\begin{array}{c}\text { Growth rate } \\
\text { in } 6 \%-9 \% \\
\text { ethanol }\end{array}$ & - & $\begin{array}{l}\text { (Bandara, } \\
\text { Fraser et } \\
\text { al. 2009) }\end{array}$ \\
\hline Ath1 & $\begin{array}{c}\text { Acidic } \\
\text { vacuolar } \\
\text { trehalase }\end{array}$ & Knockout & $\begin{array}{l}\text { Survival in } \\
18 \% \text { ethanol }\end{array}$ & $\sim 25 \%$ & $\begin{array}{c}\text { (Kim, } \\
\text { Alizadeh } \\
\text { et al. } \\
\text { 1996) }\end{array}$ \\
\hline Ath1 & $\begin{array}{c}\text { Acidic } \\
\text { vacuolar } \\
\text { trehalase }\end{array}$ & $\begin{array}{l}\text { Antisense- } \\
\text { mediated } \\
\text { inhibition }\end{array}$ & $\begin{array}{l}\text { Growth rate } \\
\text { in } 8 \% \text { ethanol }\end{array}$ & $\begin{array}{l}\text { half time to } \\
\text { reach max. } \\
\text { ethanol } \\
\text { production }\end{array}$ & $\begin{array}{l}\text { (Jung and } \\
\text { Park } \\
\text { 2005) }\end{array}$ \\
\hline Pro1 & $\begin{array}{l}\gamma \text {-glutamyl } \\
\text { kinase }\end{array}$ & $\begin{array}{c}\text { Substitution by } \\
\text { allele } \\
\text { PRO1 } \\
\text { D154N }\end{array}$ & $\begin{array}{l}\text { Survival in } \\
18 \% \text { ethanol }\end{array}$ & - & $\begin{array}{l}\text { (Takagi, } \\
\text { Takaoka et } \\
\text { al. 2005) }\end{array}$ \\
\hline CGTase & $\begin{array}{l}\text { Cyclodextrin } \\
\text { glucanotransfe } \\
\text { rase from } \\
\text { Bacillus } \\
\text { circulans }\end{array}$ & $\begin{array}{c}\text { Heterologous } \\
\text { expression }\end{array}$ & $\begin{array}{l}\text { Survival in } \\
6 \% \text { ethanol }\end{array}$ & - & $\begin{array}{l}\text { (Liang, } \\
\text { Wang et } \\
\text { al. 2011) }\end{array}$ \\
\hline
\end{tabular}

The balance of coenzymes $\mathrm{NAD}^{+} / \mathrm{NADH}$ in anabolism and redox reactions (Hou, Lages et al. 2009) has shown strong relationship with bioalcohol stress in S. cerevisiae. Yeast cells would enter a pseudo-starvation state under ethanol stress, which could probably due to cellular $\mathrm{NAD}^{+}$shortage and redox imbalance. $\mathrm{NAD}^{+}$is the cofactor of glyceraldehye 3-phosphate dehydrogenase (GAPDH), a key enzyme for glucose-toethanol conversion. To ameliorate the pseudo-starvation state caused by ethanol stress, rapid oxidation of NADH in glycerol synthesis was observed as compensation for $\mathrm{NAD}^{+}$consumption during GAPDH reaction (Takagi 2008, Vriesekoop, Haass et al. 2009, Stanley, Chambers et al. 2010). On the other hand, genes involved in 
NADH/NADPH regeneration (e.g. TDH1, GCY1, ALD4) are up-regulated under alcohol stress, which could be attributed to the indirect influence of sufficient NAD(P)H in maintaining cellular redox balance and efficient biosynthesis of amino acids, lipids and nucleotides (Ma and Liu 2010). Hence, the restoration of the $\mathrm{NAD}^{+} / \mathrm{NADH}$ redox balance plays its function in ethanol stress response.

L-proline is one of the most studied targets among the amino acids that contribute to ethanol tolerance. It has physiological function in inhibiting intracellular protein aggregation during folding/refolding process (Samuel, Kumar et al. 2000) and scavenging ROS under ethanol stress (Smirnoff and Cumbes 1989). Besides, the high output energy (30-ATP yield) of L-proline catabolism occurring in mitochondria is considered as primary energy and amino nitrogen resource that compensates for sustaining cell growth in the presence of ethanol stress (Verbruggen, Hua et al. 1996, Szabados and Savoure 2010). L-proline synthesis was successfully enriched by substituting PRO1 ( $\gamma$-glutamyl kinase, involved in the first two steps of L-proline synthesis) with a mutated allele and the resulting strain demonstrated better resistance towards $18 \%(\mathrm{v} / \mathrm{v})$ ethanol as compared with the wild-type (Takagi, Takaoka et al. 2005).

\subsubsection{From plasma membrane aspect}

Plasma membrane components are crucial for maintaining cell membrane integrity and function against bioalcohol stress. Short-chain alcohols disturb the ternary bilayer by interacting with lipid matrix through hydrophilic interactions, which include hydrogen bonds and weak hydrophobic van der waals attraction between the short hydrocarbon group and upper chain segments (Feller, Brown et al. 2002). This would 
lower membrane interfacial tension in a concentration-dependent manner that progressively leads to lipid bilayer thinning and increased fluidity (Vanegas, Contreras et al. 2012). Unsaturated oleic acid (C18:1n-9) plays a compensatory role in reducing the fluidizing effects caused by alcohols (You, Rosenfield et al. 2003, Zara, Bardi et al. 2008, Henderson and Block 2014). Moreover, a recent report has shown that total unsaturated fatty acid content rather than the degree of unsaturation is more important for bioalcohol resistance (Kim, Kim et al. 2011). The increased unsaturated fatty acids content in plasma membrane could enhance membrane integrity and stability, mitigating the fluidity caused by alcohol stress. This effect would further increase the selective absorption of substances (i.e., maltose, glucose and amino acids et al.) and lower the leakage of intracellular substances (i.e., coenzymes and cofactors et al.), finally leading to higher strain survival under alcohol stress (Stanley et al. 2010). Overexpression the genes involved in unsaturated fatty acid synthesis has improved alcohol tolerance. Overexpression of $O L E 1$ (encoding $\triangle 9$ fatty acid desaturase that introduces a double bond between carbon 9 and 10 of saturated FAs stearic acid (C18:0)) would lead to a 1.5-fold increase in C18:1n-9 degree and therefore enhanced cell viability in $10 \%(\mathrm{v} / \mathrm{v})$ ethanol (Table 7$)$. Another group had attempted to make C18:1n9 as predominant lipid in the lipid bilayer by introducing rat elongase 2 (rELO2) that elongates $\mathrm{C} 16: 0$ to $\mathrm{C} 18: 0$. The introduction of rELO2 helped increase $\mathrm{C} 18: 1 \mathrm{n}-9$ content from $30.6 \%$ to $44 \%$. The engineered yeast strain also acquired improved tolerance towards ethanol, $n$-butanol, $n$-propanol and 2-propanol (Yazawa, Kamisaka et al. 2011). Table 7. Examples of plasma membrane-based engineering approaches to improve S. cerevisiae 
bioalcohol tolerance

\begin{tabular}{|c|c|c|c|c|c|c|}
\hline Bioalcohol & Target & $\begin{array}{c}\text { Target } \\
\text { Description }\end{array}$ & $\begin{array}{c}\text { Genetic } \\
\text { Manipulation }\end{array}$ & $\begin{array}{c}\text { Phenotype } \\
\text { improvement } \\
\text { in alcohol \% } \\
(\mathrm{v} / \mathrm{v})\end{array}$ & $\begin{array}{l}\text { Production } \\
\text { Improvement }\end{array}$ & Reference \\
\hline \multirow[t]{4}{*}{ Ethanol } & Ole1 & $\begin{array}{c}\Delta 9 \text { fatty } \\
\text { acid } \\
\text { desaturase }\end{array}$ & Overexpression & $\begin{array}{l}\text { Survival in } \\
10 \% \text { ethanol }\end{array}$ & - & $\begin{array}{c}\text { (Kim, Kim } \\
\text { et al. } \\
\text { 2011) }\end{array}$ \\
\hline & rELO2 & $\begin{array}{c}\text { Rat } \\
\text { elongase } 2\end{array}$ & $\begin{array}{c}\text { Heterologous } \\
\text { expression }\end{array}$ & $\begin{array}{c}\text { Growth rate } \\
\text { in } 8 \%\end{array}$ & - & $\begin{array}{l}\text { (Yazawa, } \\
\text { Kamisaka }\end{array}$ \\
\hline & & & & ethanol & & $\begin{array}{l}\text { et al. } \\
\text { 2011) }\end{array}$ \\
\hline & Ino1 & $\begin{array}{l}\text { Inositol 1- } \\
\text { phosphate } \\
\text { synthase }\end{array}$ & Overexpression & $\begin{array}{c}3.42 \text {-fold } \\
\text { growth rate } \\
\text { in } 5 \%\end{array}$ & 1.7-fold & $\begin{array}{l}\text { (Hong, } \\
\text { Lee et al. } \\
2010)\end{array}$ \\
\hline$n$-Propanol & rELO2 & $\begin{array}{c}\text { Rat } \\
\text { elongase } 2\end{array}$ & $\begin{array}{c}\text { Heterologous } \\
\text { expression }\end{array}$ & $\begin{array}{l}\text { ethanol } \\
\text { Growth rate } \\
\text { in } 2 \% n- \\
\text { propanol }\end{array}$ & - & $\begin{array}{c}\text { (Yazawa, } \\
\text { Kamisaka } \\
\text { et al. } \\
\text { 2011) }\end{array}$ \\
\hline 2-Propanol & rELO2 & $\begin{array}{c}\text { Rat } \\
\text { elongase } 2\end{array}$ & $\begin{array}{c}\text { Heterologous } \\
\text { expression }\end{array}$ & $\begin{array}{c}\text { Growth rate } \\
\text { in 6\% 2- } \\
\text { propanol }\end{array}$ & - & $\begin{array}{c}\text { (Yazawa, } \\
\text { Kamisaka } \\
\text { et al. } \\
\text { 2011) }\end{array}$ \\
\hline$n$-Butanol & rELO2 & $\begin{array}{c}\text { Rat } \\
\text { elongase } 2\end{array}$ & $\begin{array}{c}\text { Heterologous } \\
\text { expression }\end{array}$ & $\begin{array}{l}\text { Growth rate } \\
\text { in } 1.3 \% n- \\
\text { butanol }\end{array}$ & - & $\begin{array}{c}\text { (Yazawa, } \\
\text { Kamisaka } \\
\text { et al. } \\
\text { 2011) }\end{array}$ \\
\hline Isobutanol & Ino 1 & $\begin{array}{l}\text { Inositol 1- } \\
\text { phosphate }\end{array}$ & Overexpression & $\begin{array}{l}\text { Viability in } \\
0.6 \%(\mathrm{w} / \mathrm{v})\end{array}$ & - & $\begin{array}{l}\text { (Hong, } \\
\text { Lee et al. }\end{array}$ \\
\hline & & synthase & & isobutanol & & 2010) \\
\hline
\end{tabular}

The proton-pumping ATPase amidst the bilayer can generate proton gradient to ameliorate the acidity caused by bioalcohol (Mizoguchi and Hara 1998). This proton gradient along with potassium gradient makes up for a dominant portion of yeast electrical membrane potential, which can power many cellular exchange processes 
during nutrient and waste transport, whereas elevated alcohol level (ethanol, propanol, butanol) intrigues the gradient dissipation that could agitate cell survival (Yenush, Merchan et al. 2005). Lam et al. recently bolstered the gradient by overexpressing plasma membrane proton pump (Pma1) together with plasma membrane potassium (Trk1) and conferred laboratory strain S288C over 30\% enhancement in net fermentation viability (Lam, Ghaderi et al. 2014). Meanwhile, to facilitate plasma membrane $\mathrm{H}^{+}$-ATPase activity that strongly correlates with inositol-containing glycerophospholipids (PI) degree (Furukawa, Obata et al. 2004), expression augment of Ino1 (inositol 1-phosphate synthase that catalyzes glucose-6-phosphate into inositol 1-1-phosphate) had enhanced PI precursor synthesis. The engineered strain demonstrated dramatic increase $(\sim 340 \%)$ in specific growth rate in $5 \%(\mathrm{v} / \mathrm{v})$ ethanol and increased cellular viability in $0.6 \%(\mathrm{w} / \mathrm{v})$ isobutanol (Hong, Lee et al. 2010).

\subsubsection{Other mechanism-based approaches}

Besides the mechanism and approaches discussed above, there are other methods to improve yeast bioalcohol tolerance (Table 8). Alcohol toxicity is related with ROS production in yeast mitochondria, which is regarded as one of the major alcohol damaging effects (Costa, Amorim et al. 1997). To cope with this, antioxidant Nacetyltransferase Mpr1 was overexpressed in MPR1-deficient S288C strain to acetylate toxic metabolites involved in ROS production (Du and Takagi 2007) and the resulting strain showed 50-90\% decrease in ROS level and > 3-fold survival improvement in 9\% (v/v) ethanol. In addition, Btn2 is a native yeast protein controlling subcellular localization of proteins, mediating ion, $\mathrm{pH}$ homeostasis as well as regulating 
intracellular amino acid traffic (Kama, Robinson et al. 2007, Wickner, Bezsonov et al. 2014). Btn2 overexpression had rendered yeast cells better ethanol tolerance, hypothetically due to its role in facilitating amino acid transport and protein localization under ethanol stress (Espinazo-Romeu, Cantoral et al. 2008), which suggests the key role of amino acid homeostasis in regulating ethanol resistance.

Table 8. Examples of other mechanism-based engineering approaches to improve S. cerevisiae bioalcohol tolerance

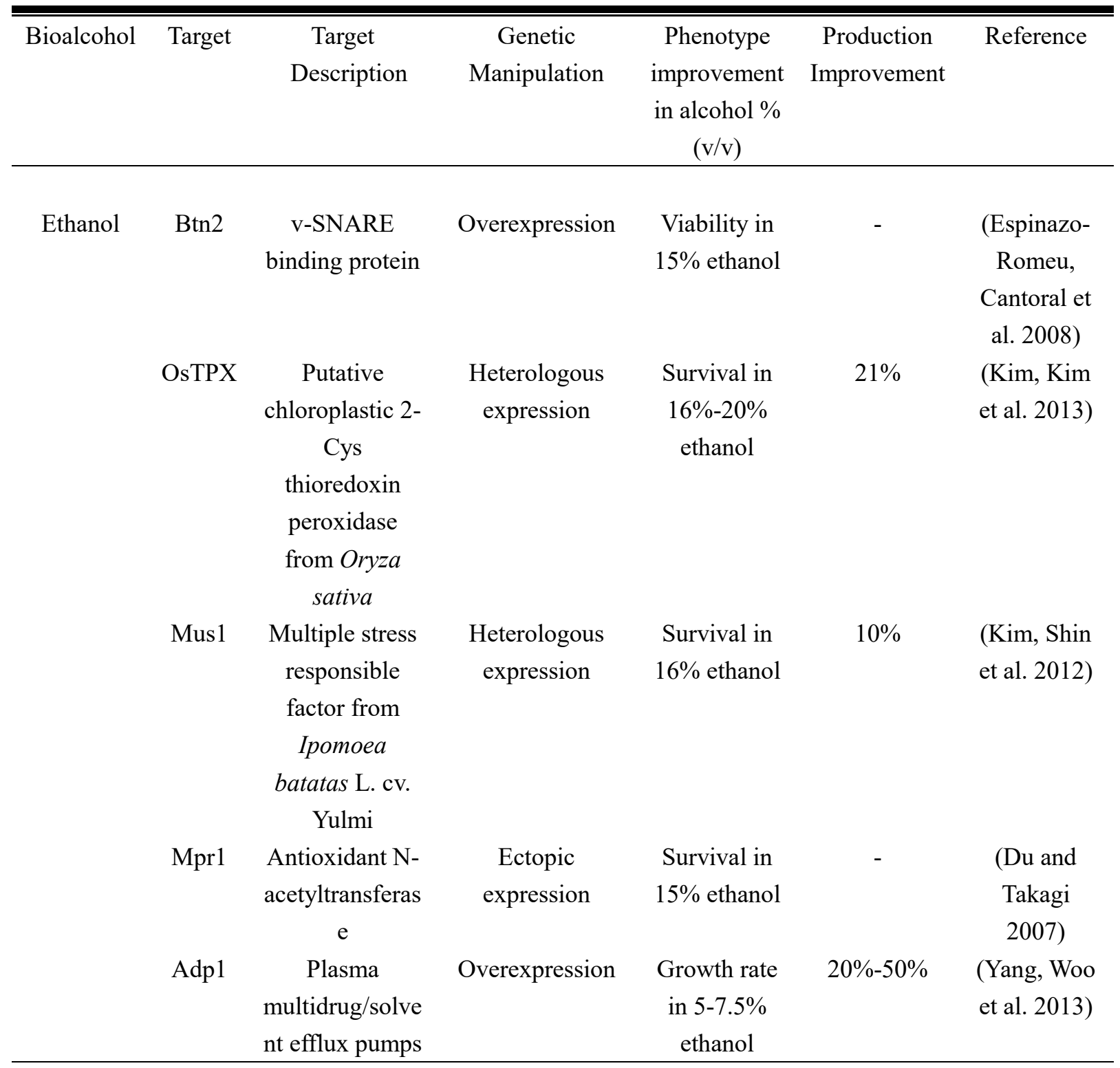




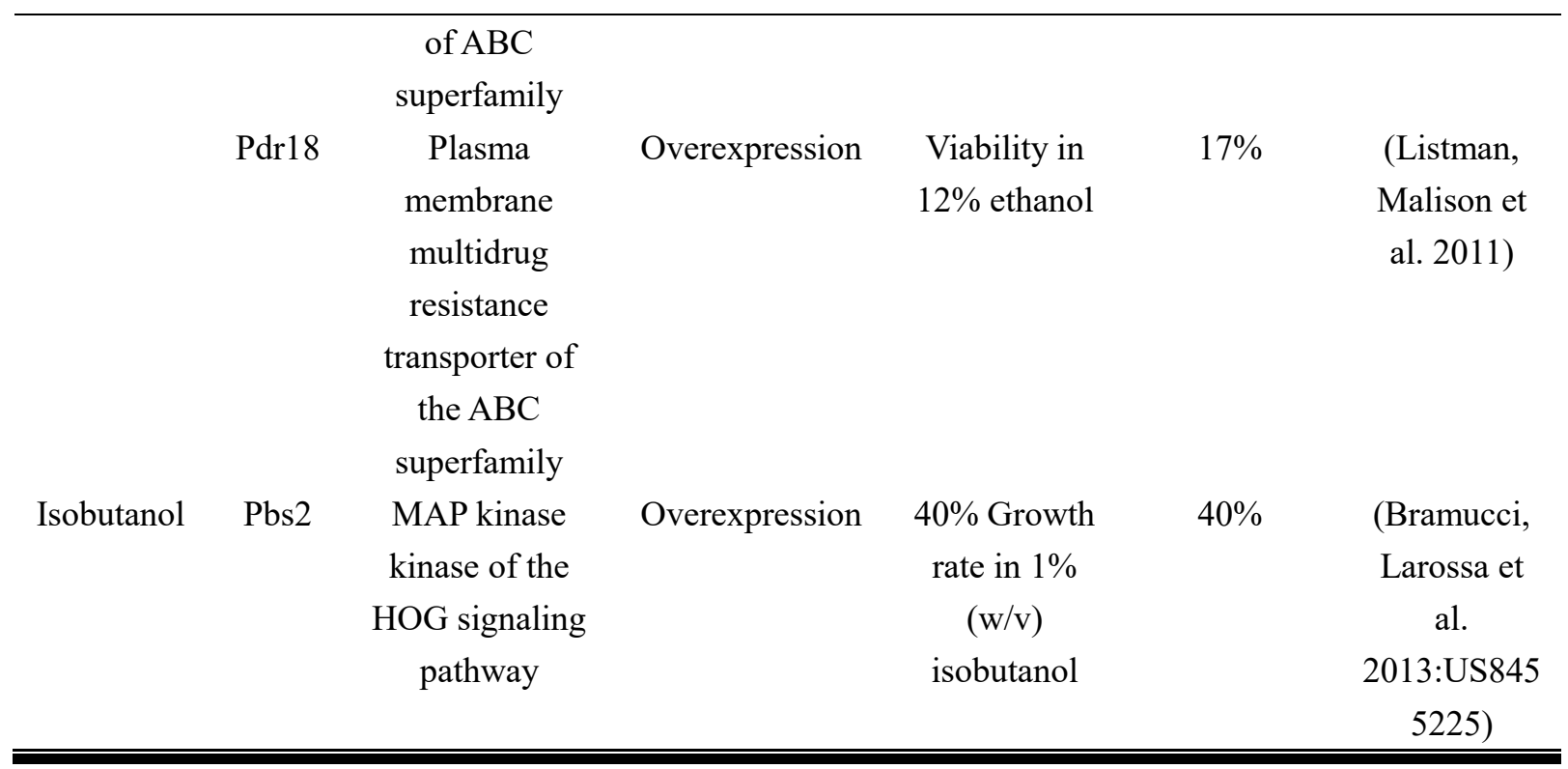

Since physiological machinery against bioalcohol stress is universal among different species, heterologous expression of regulation factors can also help enhance yeast performance under stress. For example, introducing OsTPX (putative chloroplastic 2-Cys thioredoxin peroxidase) from Oryza sativa subsp. japonica or a multiple stress responsible regulator Mus1 from sweet potato endowed yeast resistance towards ethanol stress by facilitating redox homeostasis (Kim, Shin et al. 2012, Kim, Kim et al. 2013). Compared with incorporating a bioalcohol production pathway into an organism that does not produce alcohol natively (Si, Luo et al. 2014), introducing foreign alcohol-withstanding system into intrinsically alcohol-producing strain is much easier to carry out.

Transporter engineering is an effective approach to eliminate ethanol toxic pressure by utilizing efflux pumps to export alcohols. These efflux pumps can be natively overexpressed or introduced from other species. Selected from the pool of ATP-Binding Cassette (ABC) superfamily, Adp1 (plasma multidrug pump) and Pdr18 (solvent efflux 
pump) were overexpressed, leading to $\sim 25 \%$ growth rate enhancement in $7 \%$ ethanol $(\mathrm{v} / \mathrm{v})$ and decreased susceptibility towards $12 \%(\mathrm{v} / \mathrm{v})$ ethanol stress respectively (Listman, Malison et al. 2011, Yang, Woo et al. 2013). The enhanced transporters expression facilitates the direct efflux of alcohol across the yeast. This effect would restrain plasma membrane permeabilization and finally enhance the membrane function under alcohol stress, i.e. nutrient substance intake and toxin export, finally leading to increased yeast cells survival. Compared with other rational engineering methods, transporter engineering demonstrated much better outcome, which probably is due to its particular bioalcohol ameliorating mechanism by substantially removing intracellular toxins.

In summary, the major cellular toxicities of ethanol include the inhibition of glucose and amino acid uptake, a reduction of enzyme activity participated in energy metabolism, and a disruption of membrane integrity. Ethanol is considered as the major stress responsible for the decreased ethanol production and stuck fermentation. The decreased membrane integrity of yeast cell caused by high concentration of ethanol $(>8 \%(\mathrm{v} / \mathrm{v}))$ leads to the perturbation of cell homeostasis on several cellular metabolic pathways, finally decreasing cell ethanol production capability. However, in the ethanol industry, ethanol concentration in the broth is usually over $10 \%$ (v/v) (F.W.Baia, W.A.Andersona et al. 2008). Hence, increasing yeast tolerance to ethanol become one of the main strategies in improving ethanol production.

\subsection{Combinatorial engineering}

Although the aforementioned toxicity/tolerance mechanism-based rational 
modifications of stress-relevant factors are straightforward, they still have limits: i) a systematic mechanism of bioalcohol toxicity/tolerance in yeast is not completely clarified; ii) bioalcohol tolerance trait is under polygenetic control and the result of thousands of gene interaction and gene products, which increases strain engineering difficulty. A recent global genotype-phenotype map (GPM) indicates that most traits in eukaryotes are more likely influenced by small-effect genes, for instance, distal genes whose kinetic and regulatory interactions would greatly affect strain tolerance but without clear mechanism demonstrated (Ho and Zhang 2014). In addition, to address the fundamental complexity in eliciting improved phenotype, engineering strategies call for spontaneous modulation of thousands of genes expression changes and metabolic pathway shifts, which narrows the potential of classical approaches that merely depend on mechanism background (Bailey 1999).

Following the principle of inversed metabolic engineering, first proposed by James E. Bailey (Bailey, Sburlati et al. 2002), combinatorial engineering strategies have started to attract attention in strain engineering in recent years as most of the detailed mechanism information remain uncharacterized. A simplistic diagram illustrating major combinatorial engineering strategies to improve alcohol tolerance in $S$. cerevisiae is illustrated in Figure 16. 


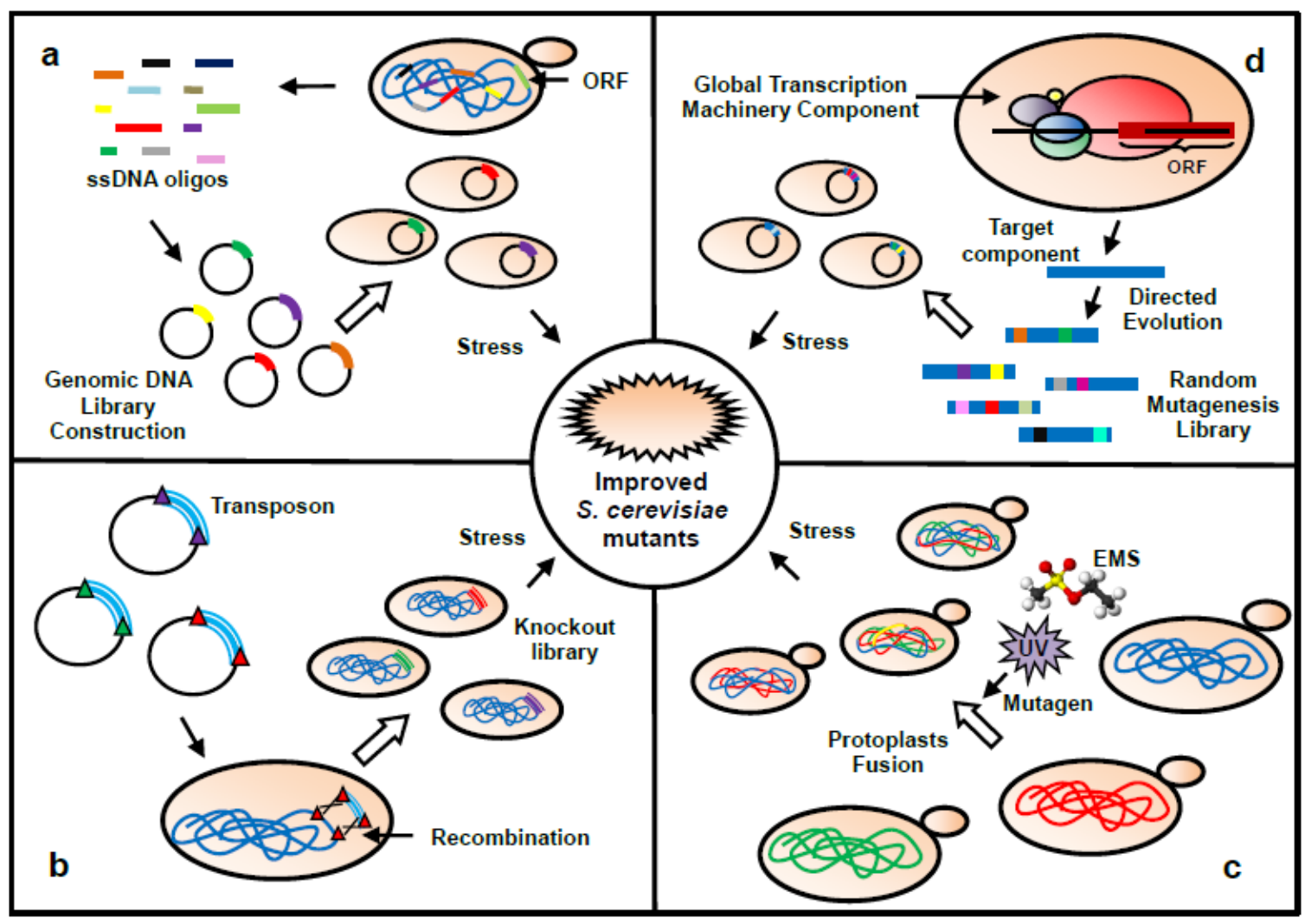

Figure 16. Diagram of the combinatorial engineering approaches implemented in S. cerevisiae to

improve alcohol tolerance. a) Random overexpression. DNA fragments from the genome are introduced into plasmids to construct a genomic DNA library. Transformants containing overexpressed genes are subjected to alcohol stress and improved mutants are isolated. b) Random knockout. The library is generated by stochastically inserting transposons that bear selecting markers into genome. Mutants showing improved tolerance towards alcohol will be studied. c) Genome shuffling. Genomic fragments from different parental strains recombine at homologous religions. Newly generated genome will be incorporated into cells by recursive protoplast fusion technology. Some groups also adopted UV/chemical mutagen pretreatment in pursuit of higher mutation frequency. d) Global transcription machinery engineering. Random mutagenesis library is obtained after directed evolution of the selected global transcription machinery targets. The library is challenged by alcohol stress and mutants demonstrate improved alcohol tolerance are isolated. 


\subsubsection{Random overexpression/knockout}

Random overexpression/knockout approach is effective in search of optimal alcohol-tolerant isolates. The success of these approaches intensively relies on: i) a priori knowledge linked to toxicity/tolerance mechanism for predicting the overexpression/knockout targets; ii) potent recombinant DNA techniques to construct libraries harboring random genetic variants; iii) high-throughput methods for selecting variants with substantial phenotype changes. Compared with evolutionary engineering, random overexpression/knockout approach possesses its advantages in acquiring geneautonomous new phenotypes. A collection of examples of random overexpression/knockout approaches is listed in Table 9.

Table 9. Examples of random overexpression/knockout approaches to improve S. cerevisiae bioalcohol tolerance

\begin{tabular}{|c|c|c|c|c|c|c|}
\hline Bioalcohol & Target & $\begin{array}{c}\text { Target } \\
\text { Description }\end{array}$ & $\begin{array}{c}\text { Genetic } \\
\text { manipulatio } \\
\mathrm{n}\end{array}$ & $\begin{array}{l}\text { Phenotype } \\
\text { improvement in } \\
\text { alcohol \% (v/v) }\end{array}$ & $\begin{array}{c}\text { Alcohol } \\
\text { Productio } \\
\text { n } \\
\text { Improve } \\
\text { ment }\end{array}$ & Reference \\
\hline \multirow[t]{4}{*}{ Ethanol } & Gal6 & $\begin{array}{l}\text { Aminopeptid } \\
\text { ase in } \\
\text { cysteine } \\
\text { protease }\end{array}$ & Deletion & $\begin{array}{c}\text { 3.35-fold survival } \\
\text { ratio in } 10 \% \\
\text { ethanol }\end{array}$ & 1.82-fold & $\begin{array}{c}\text { (Yazawa, } \\
\text { Iwahashi et al. } \\
\text { 2007) }\end{array}$ \\
\hline & Ura7 & $\begin{array}{c}\text { CTP } \\
\text { synthetase }\end{array}$ & Deletion & $\begin{array}{c}\text { Growth rate in } \\
8 \% \text { ethanol }\end{array}$ & 1.65-fold & $\begin{array}{c}\text { (Yazawa, } \\
\text { Iwahashi et al. } \\
\text { 2007) }\end{array}$ \\
\hline & Pde2 & $\begin{array}{l}\text { High-affinity } \\
\text { cAMP } \\
\text { phosphodiest } \\
\text { erase }\end{array}$ & $\begin{array}{c}\text { Overexpres } \\
\text { sion }\end{array}$ & $\begin{array}{c}\text { Survival in } 7 \% \\
\text { ethanol }\end{array}$ & - & $\begin{array}{l}\text { (Avrahami- } \\
\text { Moyal, Braun } \\
\text { et al. 2012) }\end{array}$ \\
\hline & Ssd1-V & $\begin{array}{l}\text { The truncated } \\
\text { SSD1, SSD1- }\end{array}$ & $\begin{array}{l}\text { Overexpres } \\
\text { sion }\end{array}$ & $\begin{array}{c}\text { Survival in } 7 \% \\
\text { ethanol }\end{array}$ & - & $\begin{array}{l}\text { (Avrahami- } \\
\text { Moyal, Braun }\end{array}$ \\
\hline
\end{tabular}




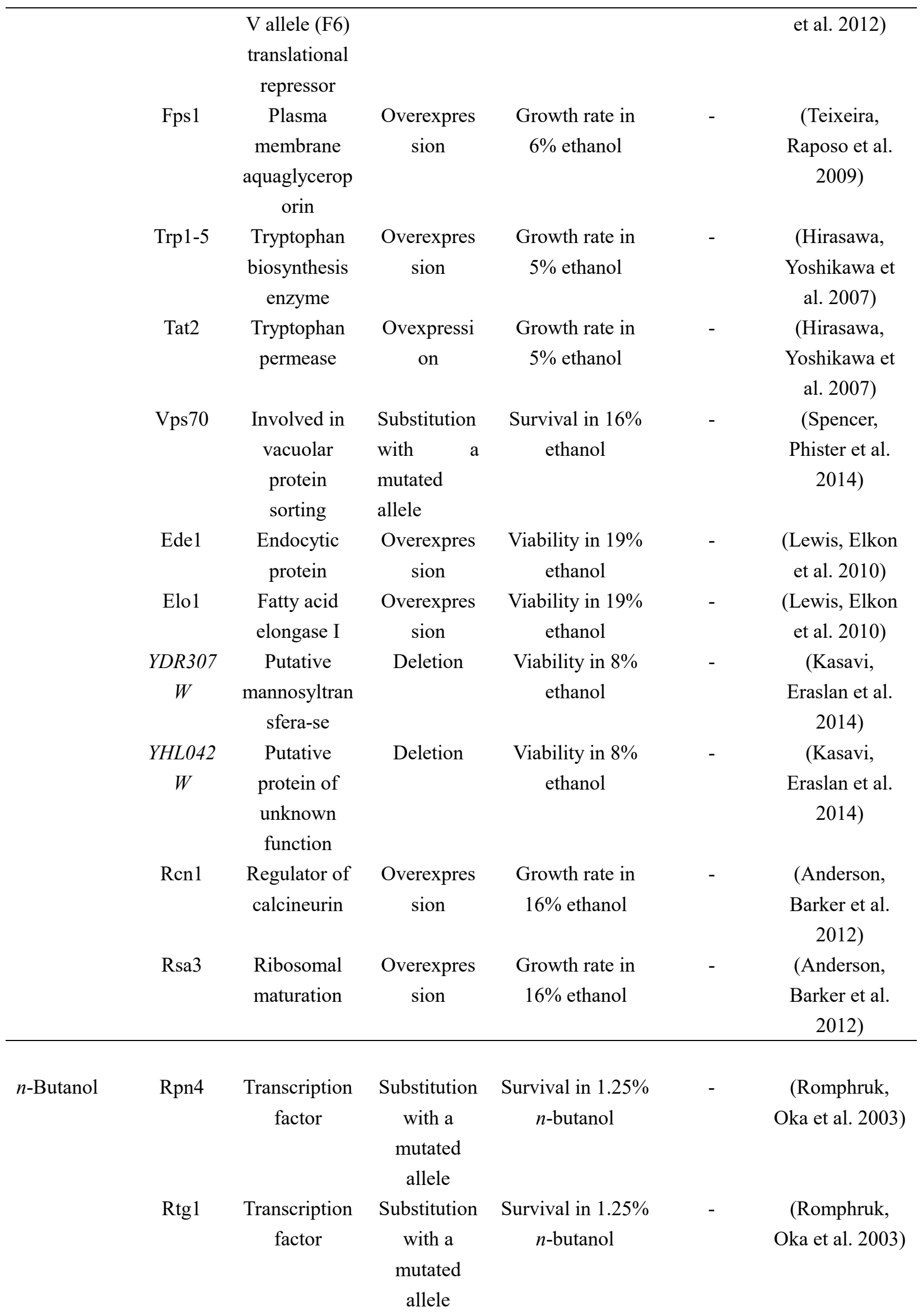




\begin{tabular}{|c|c|c|c|c|c|c|}
\hline \multirow[t]{2}{*}{ Isobutanol } & Dog1 & $\begin{array}{c}\text { 2- } \\
\text { deoxyglucose } \\
\text {-6-phosphate } \\
\text { phosphatase }\end{array}$ & $\begin{array}{l}\text { Overexpres } \\
\text { sion }\end{array}$ & $\begin{array}{l}\text { Survival in } 1 \% \\
\text { isobutanol }\end{array}$ & - & $\begin{array}{c}\text { (Honga, Leea et } \\
\text { al. 2010) }\end{array}$ \\
\hline & Hall & $\begin{array}{l}\text { Cytoplasmic } \\
\text { protein }\end{array}$ & $\begin{array}{l}\text { Overexpres } \\
\text { sion }\end{array}$ & $\begin{array}{c}\text { Survival in } 1 \% \\
\text { isobutanol }\end{array}$ & - & $\begin{array}{c}\text { (Honga, Leea et } \\
\text { al. 2010) }\end{array}$ \\
\hline
\end{tabular}

Random overexpression libraries can be obtained from endogenous or exogenous yeast genome. For example, after random overexpression of $S$. cerevisiae autologous genomic fragments, isolated mutants bearing increased level of Dog1 (2-deoxyglucose6-phosphate phosphatase), Hall (cytoplasmic protein involved in halotolerance) and a truncated Msn2 exhibited improved tolerance towards ethanol and isobutanol respectively (Honga, Leea et al. 2010). Similar approach was employed by introducing genomic library prepared from ethanol-tolerant strain S288C into ethanol-sensitive strain W303-1A (Anderson, Barker et al. 2012). It was found that increased dosage of either Rcn1 (calcineurin regulator) or Rsa3 (involved in ribosome maturation) would lead to improved recovery after $16 \%$ (v/v) ethanol shock, which suggests that the regulation of ribosome biogenesis and calcineurin signaling pathway is critical for $S$. cerevisiae survival under ethanol stress.

Random knockout libraries are either commercially available or generated via transposon mutagenesis by means of randomly integrating antibiotic or amino acid synthesis marker cassettes into genome regions (Takahashi, Shimoi et al. 2001). The screening of random knockout libraries has assisted the identification of important alcohol stress-relevant genes, including TRP1, TRP2, TRP3, TRP4, TRP5 (tryptophan biosynthesis genes) (Hirasawa, Yoshikawa et al. 2007) and FPS1 (controlling glycerol 
efflux) (Teixeira, Raposo et al. 2009). The overexpression of these genes demonstrated improved cell growth in 5-6\% (v/v) ethanol. By comparison, some recent techniques on regulating gene expression are more flexible in repressing gene expression. For example, Lim group has recently generated a RNA-guided DNA recognition platform to reversibly repress target gene expression in E. coli (Qi, Larson et al. 2013). Catalytically dead Cas9 (a RNA-guided DNA endonuclease from typical II CRISPR system) lacking endonuclease activity was co-expressed with guide RNAs to generate a DNA recognition complex that can specifically interfere transcriptional elongation, RNA polymerase binding, and transcription factor binding. In addition, synthetic small regulatory RNAs (sRNAs) were also designed for target gene knockdown to elicit improved phenotypes such as production of tyrosine and cadaverine in E. coli (Na, Yoo et al. 2013). With the help of these techniques, researchers will be able to perturb gene expression selectively without library construction or chromosomal modulation in $S$. cerevisiae.

\subsubsection{Genome shuffling}

In vitro genome shuffling combined with artificial recursive protoplast fusion is a powerful way to rapidly propagate bioalcohol-tolerant strains. The purpose of this approach is to achieve greater genetic diversity, i.e. to expand genomic background to elicit desired phenotype. Various attempts had been made to achieve genetic variation via different mutagenesis methods, including pretreating protoplast by ultraviolet irradiation (Shi, Wang et al. 2009), high-energy pulse electron beam (HEPE) (Zhang, Xiao et al. 2012), chemical mutagens (Liu, Ding et al. 2011), and error-prone genome 
amplification (Luhe, Tan et al. 2011). Besides, homologous genome recombination can be achieved by adopting native strain (Zheng, Chen et al. 2014) or different donors (Shi, Wang et al. 2012). Shi et al. transferred short chromosome DNA fragments from different ethanol-tolerant parental strains into an acceptor cell and obtained a mutant strain with $>4$-fold survival improvement in $13 \%(v / v)$ ethanol. Compared with single gene moderation, genome shuffling offers the advantage by spontaneously introducing genetic changes at multiple positions throughout the whole genome. Nevertheless, the effectiveness of this approach has been questioned as multiple rounds of shuffling may intrigue the loss of existing beneficial properties since the chance of generating new improved mutations equals to the chance of losing existing advantageous genomic fragments.

\subsubsection{Global transcription machinery engineering (gTME)}

Global transcription machinery engineering (gTME) is able to reprogram cellular transcription to elicit phenotypes valuable for industry applications through fine-tuning of global transcription machinery components (Alper and Stephanopoulos 2007). The components selected so far are critical proteins regulating global transcriptome, including zinc finger-containing artificial transcription factors (Park, Lee et al. 2003), global/general transcription factors (Alper, Moxley et al. 2006), exogenous regulator from Deinococcus radiodurans (Chen, Wang et al. 2011, Wang, Zhang et al. 2012) and native global regulator Hha/H-NS (Hong, Wang et al. 2010, Hong, Hegde et al. 2012) and cAMP receptor protein (CRP) (Zhang, Chong et al. 2012, Chong, Geng et al. 2014) in E. coli. 
Compared with classical strain engineering, gTME has shown its advantage in generating high degree of pleiotropy. Klein et al. compared the introduced phenotypic diversity of Lactobacillus plantarum (L. plantarum) between gTME-based library (containing variants bearing random mutated RNA synthesis initiation factor, $\sigma$-factor rpoD of L. plantarum ) and chemical mutagen NTG-based (N-methyl-N-nitro-Nnitrosoguanidine) library (Klein-Marcuschamer and Stephanopoulos 2008), and broader distribution of the average phenotypic distance was observed in gTME library, which implies its superior efficiency to endow organism phenotype variation. Moreover, mutant selection period can be greatly shortened to just 3 5 days, as compared to months/years using classical strain engineering methods.

gTME has been successfully exploited in $S$. cerevisiae to improve strain ethanol tolerance. TATA-binding protein (Spt15) is in charge of the binding of transcription initiation complex to promoter sequence (TATA box motif). Alper et al. had introduced random mutations into SPT15 gene through error-prone PCR and isolated a mutant with over 12-fold tolerance improvement in $6 \%(\mathrm{v} / \mathrm{v})$ ethanol environment (Alper, Moxley et al. 2006). It deserves attention that Spt15 had been further engineered to improve yeast cell tolerances to hyperosmotic (Kim, Yang et al. 2013) and oxidative stress (Zhao, Li et al. 2014). This capacity of one target mutagenesis to induce several improved phenotypes is also observed in other transcription machinery components such as CRP (Basak and Jiang 2012, Basak, Song et al. 2012). Hence it is possible to obtain a mutant with multiple improved phenotypes in gTME. 


\subsubsection{Adaptive evolution \& hybridization}

In addition to combinatorial engineering approaches, adaptive evolution and hybridization are also simple and direct random engineering ways to improve yeast tolerance. Driven by spontaneous or induced mutagenesis, adaptive monoclonal population can increase in continuous cultivation with accelerating bioalcohol level and competitive strains can be selected. Such process has been extensively executed by several groups to evolve yeast strains with enhanced ethanol (Dinh, Nagahisa et al. 2008, Stanley, Fraser et al. 2010, Thammasittirong, Thirasaktana et al. 2013, Chen and Xu 2014) and butanol tolerance (Ghiaci, Norbeck et al. 2013). Two factors would determine the success of this continuous culturing evolution: i) appropriate mutation rate (Manrubia, Escarmis et al. 2005); ii) suitable selection pressure (Cakar, Seker et al. 2005). In addition to using traditional mutagens such as UV or ethyl methane sulfonate (EMS), a novel effective mutation technique has been adopted in evolution engineering. Since non-lethal genomic mutation occurs preferentially in the phase of lagging-strand synthesis during DNA replication, a proofreading-deficient DNA polymerase $\delta$ (pol31, involved in the ligation of Okazaki fragments that increases the mutation rate during lagging-strand synthesis phase) was introduced into $S$. cerevisiae and acted as strong mutagen (Abe, Fujita et al. 2009). An optimal strain was isolated with superior viability in $10 \%(\mathrm{v} / \mathrm{v})$ ethanol.

Another evolution approach is hybridization through crossing strains of different background in pursuit of broader genetic diversity. Benítez group previously generated a large number of wine-laborotary yeast strain hybrids subjected to cultures with 
increasing ethanol concentration (Jiménez and Benítez 1988). The optimal strain isolated had viability at lethal ethanol concentration $(16 \%(\mathrm{v} / \mathrm{v}))$. Benjaphokee et al. gained an evolved strain resistant towards $15 \%(\mathrm{v} / \mathrm{v})$ ethanol via a similar spore-to-cell hybridization approach (Benjaphokee, Hasegawa et al. 2012).

However, adaptive evolution \& hybridation encompass recombination and continous evolution of large cell population over rounds of subcultures $(>100$ generations), which may take months/years to breed an optimal strain. The detailed mutation information in the isolated strains also requires expensive genome sequencing. In addition, the apperance of silent mutations during these empirical engineering is difficult to filter out (Jong, Siewers et al. 2012).

\subsection{Conclusions and future prospects}

Dissections of genome and epigenome, transcription, translation, metabolism as well as plasma membrane systems have presented a comprehensive landscape to illustrate bioalcohol toxicity/tolerance mechanism in S. cerevisiae. But the scope of mechanism is still expected to be expanded with new information obtained from newly developed mutants, which will help illustrate how alcohols damage yeast cells and what cellular responses are against such damages. We believe that the advances in "-omics" and system biology would promote to establish the mechanism dynamics in $S$. cerevisiae that can make delicate multi-genic engineering possible. On the other hand, combinatorial engineering and adaptive evolution \& hybridization approaches have been developed following evolution strategies. Some recently exploited high throughput screening technology such as the use of microfluid systems would help to 
greatly shorten mutant isolation period. For example, Gregory Stephanopoulos group has reported one high-throughput approach that uses microfluidics to compartmentalize individual cells for growth and analysis in monodisperse nanoliter aqueous droplets surrounded by an immiscible fluorinated oil phase (Wang et al. 2014). Then, a fluorescence assay was applied based on the pyranose oxidase enzyme to detect xylose concentration in each cell's droplet to identify high xylose consumption yeast. This approach may fulfil the requirement for high-throughput single cell sorting and phenotype characterization for mutants library.

\subsection{Acknowledgements}

This work is supported by National Research Foundation (NRF-CRP-5-2009-03) and Ministry of Education (MOE2012-T2-2-117), Singapore. 


\section{Chapter 5. Improving fatty acids production through enhancing the precursor malonyl-CoA supply in Saccharomyces cerevisiae}

\subsection{Introduction}

In addition to bio-alcohols, $S$. cerevisiae has also been exploited to produce biofuels with more energy. As for the generation of renewable fuels and chemicals, the high energy-rich molecules, fatty acids and their derivatives, are considered ideal advanced biofuels to replace fossil fuels since they are excellent candidates for renewable liquid transport fuels and chemicals (Zhang, Rodriguez et al. 2011, Lian and Zhao 2015). Among various microbes, S. cerevisiae has been extensively investigated as one of the most popular platforms for producing fatty acids due to its well-studied genetics and physiological background, less possibility of phage contamination, compatibility of large-scale fermentation, and high resistance towards toxic inhibitors and products (Hong and Nielsen 2012). However, one major challenge for fatty acids production in S. cerevisiae is the insufficient supply of precursor malonyl-CoA (van Roermund, Waterham et al. 2003, Tehlivets, Scheuringer et al. 2007).

To deal with this problem, extensive metabolic engineering efforts have been made to improve malonyl-CoA supplement in S. cerevisiae. Fatty acids synthesis is initiated by the conversion of acetyl-CoA to malonyl-CoA via acetyl-CoA carboxylase (ACC) (van Roermund, Waterham et al. 2003). As the supply of acetyl-CoA in cytosol is limited, one common approach is to direct glycolytic flux towards acetyl-CoA biosynthesis through different "push and pull" strategies, including: i) overexpressing pyruvate dehydrogenase (PDH) bypass to accumulate more acetate to transfer to acetyl- 
CoA by acetyl-CoA synthetase (Remize, Andrieu et al. 2000); ii) inactivating competing pathways $(\triangle A D H 1 / 4)$ to disrupt the metabolic flux from aldehyde to ethanol (Li, Guo et al. 2014); iii) introducing heterologous ATP-dependent citrate lyase (ACL) with better efficiency to promote the transfer of cytosolic citrate to acetyl-CoA (Tang, Feng et al. 2013, Lian, Si et al. 2014); iv) inactivating acetyl-CoA consuming pathway ( $\triangle C$ CIT2, $\triangle M L S 1)$ (Chen, Daviet et al. 2013). All these strategies for improving acetylCoA level have been focused on engineering the "acetyl-" part of acetyl-CoA. Our previous work has tried to elevate acetyl CoA level from both the "-CoA" part and the "acetyl-" part, via overexpressing pantothenate kinase (Pank) from S. cerevisiae and introducing a PDH bypass (ALD6 from S. cerevisiae and SeAcs ${ }^{L 641 P}$ from Salmonella enteric). As it turned out, acetyl-CoA level was elevated by $\sim 3$-fold in S. cerevisiae, providing a valuable platform for fatty acids synthesis of this study. As for the conversion of acetyl-CoA to malonyl-CoA, simple overexpression of ACC only increase the fatty acid production by about 2-fold (Shi, Valle-Rodríguez et al. 2012). Since the activity of ACC1 was lost after the post-transcriptional phosphorylation by SNF1, mutating the putative phosphorylation sites from serine to alanine in ACC1 $\left(A C C 1^{S 659 A, S 1157 A}\right)$ has increased the ACC activity by more than 3-fold (Shi, Chen et al. 2014, Kildegaard, Jensen et al. 2016).

In addition to ACC pathway, malonyl-CoA can also be directly synthesized in $S$. cerevisiae. Overexpressing plant malonyl-CoA synthetase gene (AAE13) in $S$. cerevisiae that ligates malonic acid and CoA to generate malonyl-CoA has led to a 1.6fold increase in lipid accumulation (Wang, Chen et al. 2014). In addition, MatB from 
bacteria Rhizobium leguminosarium encoding malonyl-CoA synthetase, can directly catalyze the generation of malonyl-CoA from malonate and CoA (Kim 2002). However, S. cerevisiae cannot produce malonate naturally and uptake exogenously supplied malonate in malonic acid form. To overcome this dilemma, a heterogeneous dicarboxylic acid plasma membrane transporter MAE1 (Schizosaccharomyces pombe), which can import exogenous malonic acid, has been introduced into S. cerevisiae previously (Chen and Tan 2013).

In view of these findings, we planned to couple the engineered acetyl-CoA pathway (overexpression of PanK, $A L D 6, \mathrm{Se} A c s^{L 641 P}$ and $A C C 1^{S 659 A, S 1157 A}$ ) and malonyl-CoA synthetase pathway (introduction of $M A E 1$ and $M a t B$ in $S$. cerevisiae) to increase the supply of malonyl-CoA for fatty acids production (Figure 17). The combination of the two pathways is fulfilled by co-expressing both the plasmid with acetyl-CoA pathway and the plasmid with malonyl-CoA synthetase pathway. After $24 \mathrm{~h}$ fermentation with malonic acids, the accumulated C16:0 and C18:0 in the engineered strain with two pathways reached 86.74 and $86.97 \mathrm{ug} / 10^{8}$ cells respectively, which was about 4 -fold that of the wild-type strain. 


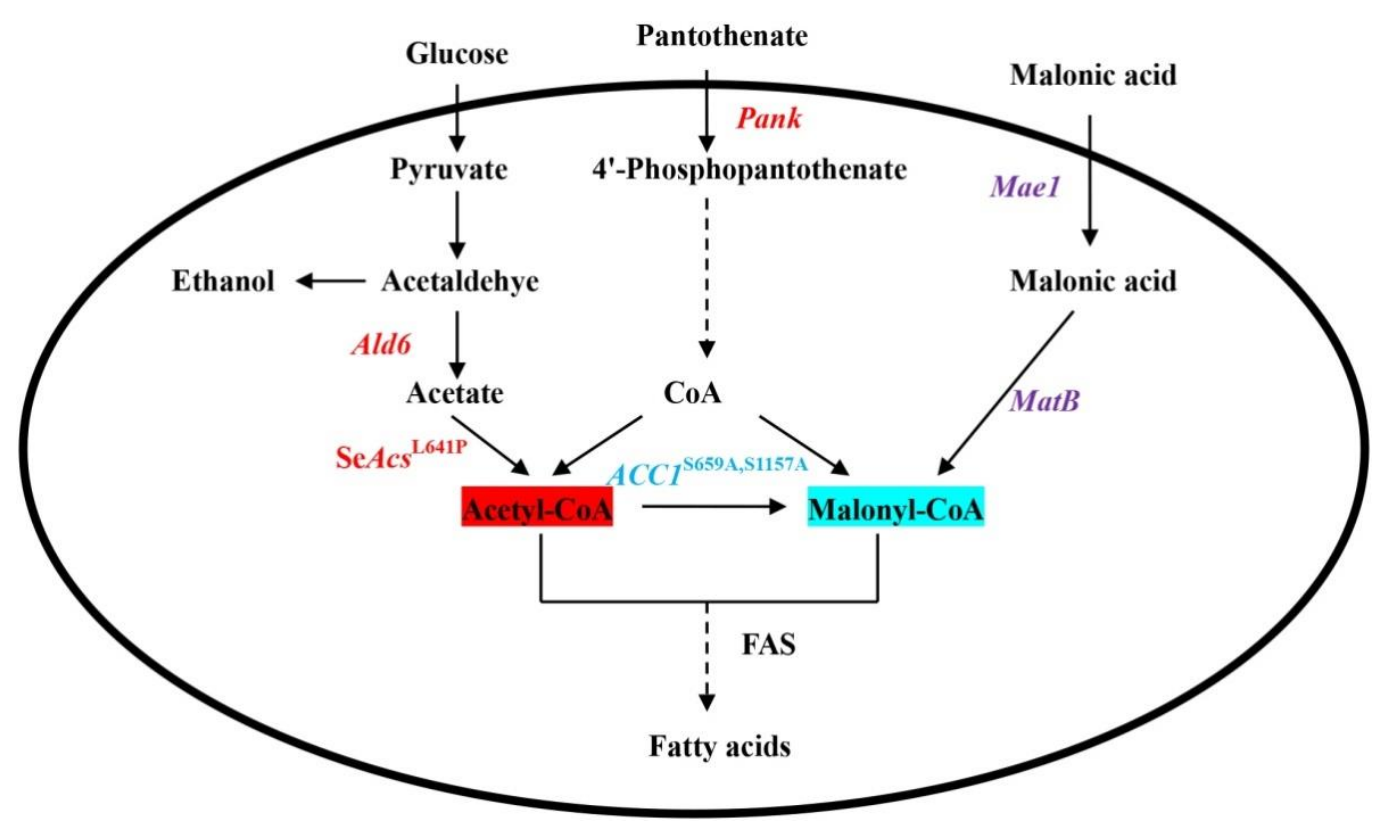

Figure 17. Simplified overview of the engineering strategy to enhance the acetyl-CoA carboxylase pathway (overexpression of PanK, $A L D 6$, Se $A c s^{L 641 P}$ and $A C C 1^{S 659 A, ~ S 1157 A}$ ) and malonyl-CoA synthetase pathway (introduction of $M A E 1$ and MatB in $S$. cerevisiae) to increase the supply of malonyl-CoA precursors for fatty acids production.

\subsection{Materials and methods}

\subsubsection{Plasmid and strains construction}

In acetyl-CoA carboxylase pathway, plasmid p426PAA containing expression cassette pRS426GAL1 with HXT7-CAB1,TEF1p-ALD6-ADH1t and TDH3pSeAcs ${ }^{L 641 P_{-}}$PGIt was acquired from our previous study (Wenshan, Bo et al. 2016). The TEF1p-ACC1 S659A, S1157A $-A D H 1 t$ cassette (all amplified from S. cerevisiae BY4742 genome) was constructed by overlap PCR. DNA fragments were assembled into XhoI and BamHI sites of the linearized p41K plasmid, which was pretreated with BamHI and XhoI, to form the new plasmid p41KC (Table 10) (Shao, Zhao et al. 2009). To construct malonyl-CoA synthesis pathway, two genes encoding dicarboxylic acid plasma 
membrane transporter (MAE1) cDNA (GenBank: U21002.1) from Schizosaccharomyces pombe and malonyl-CoA synthetase (MatB) cDNA (GenBank:AAC83455.1) from bacteriaRhizobium leguminosarium were codon optimized and synthesized by Optimal Biotech (Singapore). PGK1p-MAE1-ADH1t and TEF2p-MatB-ADH2t expression cassettes were constructed by overlap PCR using primer 1-6 and 7-12, respectively (Table 11). These two DNA fragment were assembled with the linearized $\mathrm{p} 41 \mathrm{~K}$ separately to form plasmid $\mathrm{p} 41 \mathrm{KA}, \mathrm{p} 41 \mathrm{~KB}$ and $\mathrm{p} 41 \mathrm{KAB}$ respectively (Table 10). Similarly, the constructed TEF1p-ACC1 $1^{S 659 A, S 1157 A}-A D H 1$, PGK1p-MAE1-ADH1t and TEF2p-MatB-ADH2t were amplified by PCR using primer $23 \& 24,11 \& 25,22 \& 26$ respectively and the PCR fragments were assembled into linearized $\mathrm{p} 41 \mathrm{~K}$ to form $\mathrm{p} 41 \mathrm{KABC}$. All constructed plasmids (Table 10) had been sent to DNA sequencing for confirmation and transformed into CEN.PK2-1C (EUROSCARF) to construct the engineered strains in this study (Table 12). 
Table 10. Plasmids in this study

\begin{tabular}{|c|c|c|}
\hline Name & Description & Source \\
\hline pRS426GAL1 & $(2 \mu U R A 3)$ & $\begin{array}{l}\text { (Wenshan, Bo et } \\
\text { al. 2016) }\end{array}$ \\
\hline pRS41K & kanMX & EUROSCARF \\
\hline p426РAA & $P_{H X T 7-C A B 1} P_{T E F 1}-A L D 6 \quad P_{T D H 3}-\operatorname{Se} A c s^{L 641 P}(2 \mu U R A 3)$ & This study \\
\hline $\mathrm{p} 41 \mathrm{KA}$ & PGKIp-MAE1-ADHIt (KanMX) & This study \\
\hline $\mathrm{p} 41 \mathrm{~KB}$ & $T E F 2 p-M a t B-A D H 2 t($ KanMX) & This study \\
\hline $\mathrm{p} 41 \mathrm{KAB}$ & PGK1p-MAE1-ADH1t TEF2p-MatB-ADH2t (KanMX) & This study \\
\hline $\mathrm{p} 41 \mathrm{KABC}$ & $\begin{array}{l}\text { TEF1p-ACC1 }{ }^{\mathrm{S} 659 \mathrm{~A}, \mathrm{~S} 1157 \mathrm{~A}}-A D H 1 t \text { PGK1p-MAEl-ADH1t } \\
\text { TEF2p-MatB-ADH2t (KanMX) }\end{array}$ & This study \\
\hline $\mathrm{p} 41 \mathrm{KC}$ & $T E F 1 p-A C C 1^{\mathrm{S} 659 \mathrm{~A}, \mathrm{~S} 1157 \mathrm{~A}}-A D H 1 t($ KanMX $)$ & This study \\
\hline
\end{tabular}

Table 11. Primers used in this study

\begin{tabular}{|c|c|}
\hline $\begin{array}{l}\text { Primer } \\
\text { no. }\end{array}$ & Sequence (5' to 3') \\
\hline 1 & $\begin{array}{l}\text { TACGACTCACTATAGGGCGAATTGGGTACCGGGCCCCCCCTCGAG } \\
\text { CACACACCATAGCTTCAAAATGTTT }\end{array}$ \\
\hline 2 & AATAAGCTTTCTTCGCTCATTTTGTAATTAAAACTTAGAT \\
\hline 3 & ATCTAAGTTTTAATTACAAAATGAGCGAAGAAAGCTTATT \\
\hline 4 & AATCAAAAGACCACCATCAGCTAGTTGACGCAGTATGATA \\
\hline 5 & TATCATACTGCGTCAACTAGCTGATGGTGGTCTTTTGATT \\
\hline 6 & ATATGACAAATCTGAAACAGCAACAGCCCTGTTCATACCC \\
\hline 7 & GGGTATGAACAGGGCTGTTGCTGTTTCAGATTTGTCATAT \\
\hline 8 & ACAAAGGAAAAGGGGCCTGTTTATTTCAAAGTCTTCAACA \\
\hline 9 & TGTTGAAGACTTTGAAATAAACAGGCCCCTTTTCCTTTGT \\
\hline 10 & $\begin{array}{l}\text { GCTGGAGCTCCACCGCGGTGGCGGCCGCTCTAGAACTAGTGGATCCGCAA } \\
\text { ATTAAAGCCTTCGAGC }\end{array}$ \\
\hline 11 & $\begin{array}{l}\text { TACGACTCACTATAGGGCGAATTGGGTACCGGGCCCCCCCTCGAG } \\
\text { GTGAGTAAGGAAAGAGTGAGGAACT }\end{array}$ \\
\hline 12 & ATCTCCTTCAATTCACCCATTGTTTTATATTTGTTGTAAA \\
\hline 13 & TTTACAACAAATATAAAACAATGGGTGAATTGAAGGAGAT \\
\hline 14 & ATAAATCATAAGAAATTCGCTTAAACTGATTCATGTTCAG \\
\hline 15 & CTGAACATGAATCAGTTTAAGCGAATTTCTTATGATTTAT \\
\hline 16 & $\begin{array}{l}\text { TACTATATGTAAGTATACGGCCCCAGCGGCCGCCCGTGTGGAAGAACGATT } \\
\text { ACAACAG }\end{array}$ \\
\hline
\end{tabular}




\begin{tabular}{ll}
\hline 17 & CTGTTGTAATCGTTCTTCCACACGGGCGGCCGCTGGGGCCGTATACTTACA \\
& TATAGTA \\
18 & TCGAACAAGTGGTTCGACATGTTTAGTTAATTATAGTTCG \\
19 & CGAACTATAATTAACTAAACATGTCGAACCACTTGTTCGA \\
20 & TAAAGACATAAGAGATCCGCTTACGTCCTAATGTAAAGAT \\
21 & ATCTTTACATTAGGACGTAAGCGGATCTCTTATGTCTTTA \\
22 & GCTGGAGCTCCACCGCGGTGGCGGCCGCTCTAGAACTAGTGGATCC \\
& GCAAATTAAAGCCTTCGAGC \\
23 & CTGTTGTAATCGTTCTTCCACACGGGAGCTCCACACACCATAGCTTCAAAA \\
& TGTTT \\
24 & TACTATATGTAAGTATACGGCCCCAGCGGCCGCGCAAATTAAAGCCTTCGA \\
& GC \\
25 & AAACATTTTGAAGCTATGGTGTGTGGAGCTCCCGTGTGGAAGAACGATTAC \\
& AACAG \\
& GCTCGAAGGCTTTAATTTGCGCGGCCGCTGGGGCCGTATACTTACATATAG \\
& TA
\end{tabular}

Table 12. Strains in this study

\begin{tabular}{lll}
\hline Name & Description & Source \\
CEN.PK2-1C & MATa; ura3-52; trp1-289; leu2-3,112; his3 $41 ;$ MAL2- & EUROSCARF \\
& $8 C ;$ SUC2 & \\
CEN-K-6 & CEN.PK2-1C + pRS426GAL1+pRS41K & This study \\
CEN-PAA-K & CEN.PK2-1C + p426PAA+ pRS41K & This study \\
CEN-PAA-C & CEN.PK2-1C + p426PAA+ p41KC & This study \\
CEN-PAA-AB & CEN.PK2-1C + p426PAA+ p41KAB & This study \\
CEN-PAA-ABC & CEN.PK2-1C + p426PAA+ p41KABC & This study \\
CEN-A & CEN.PK2-1C + p41KA & This study \\
CEN-B & CEN.PK2-1C + p41KB & This study \\
CEN-AB & CEN.PK2-1C + p41KAB & This study \\
\hline
\end{tabular}

\subsubsection{Media and growth conditions}

E. coli DH5 $\alpha$ was used for cloning and cultured in LB medium with $100 \mu \mathrm{g} / \mathrm{mL}$ ampicillin at $37{ }^{\circ} \mathrm{C}$. Yeast cells were cultured in YPD media $(20 \mathrm{~g} / \mathrm{L}$ peptone, $10 \mathrm{~g} / \mathrm{L}$ yeast extract and $20 \mathrm{~g} / \mathrm{L}$ glucose) at $30{ }^{\circ} \mathrm{C}$. Recombinant yeast strains were screened 
and grown in auxotrophic Complete Minimal medium (CM, $6.7 \mathrm{~g} / \mathrm{L}$ yeast nitrogen base without amino acids, $20 \mathrm{~g} / \mathrm{L}$ glucose, $150 \mathrm{mg} / \mathrm{L}$ valine, $20 \mathrm{mg} / \mathrm{L}$ adenine hemisulfate, $20 \mathrm{mg} / \mathrm{L}$ arginine-HCl, $30 \mathrm{mg} / \mathrm{L}$ lycine- $\mathrm{HCl}, 20 \mathrm{mg} / \mathrm{L}$ methionine, $200 \mathrm{mg} / \mathrm{L}$ threonine, $30 \mathrm{mg} / \mathrm{L}$ tyrosine, $50 \mathrm{mg} / \mathrm{L}$ phenylalanine, optionally supplemented with $100 \mathrm{mg} / \mathrm{L}$ leucine, $20 \mathrm{mg} / \mathrm{L}$ histidine and $20 \mathrm{mg} / \mathrm{L}$ tryptophane) supplied with $200 \mu \mathrm{g} / \mathrm{mL} \mathrm{G418}$ at $30^{\circ} \mathrm{C}$.

\subsubsection{GC-MS analysis of free fatty acids}

Strains with three biological replicates were pre-cultured in $5 \mathrm{~mL}$ aliquots in $\mathrm{CM}$ medium with $200 \mu \mathrm{g} / \mathrm{mL}$ G418 overnight and used to inoculate fresh $20 \mathrm{~mL} \mathrm{CM}$ medium (supplied with $200 \mu \mathrm{g} / \mathrm{mL}$ G418, $50 \mu \mathrm{M}$ pantothenate and $0.38 \%(\mathrm{w} / \mathrm{v})$ malonic acids) in 250-mL flask cultures to achieve initial OD 600 of 0.2 . The OD 600 of the medium were detected constantly during the culture. Same amounts of yeast cell cultures were collected by centrifugation after $24 \mathrm{~h}$ (exponential phase) and washed twice with distilled water. After three cycles of freezing $\left(-80{ }^{\circ} \mathrm{C}\right)$ and thawing, cells were then dissolved in a mixture of methanol/water extraction solvent in ratio of 5:2 (v/v) and $5 \mu \mathrm{L}$ heptadecanoic acid $(5 \mathrm{mg} / \mathrm{ml})$ was added as internal standard in the extracted solvent to correct for mechanism loss during sample preparation. The extracted solvent was subjected to vortex for 30 minutes mixing in the presence of acidwashed glass beads $(425-600 \mu \mathrm{m})$ and then added with chloroform to form a mix of chloroform/methanol/water in ratio of 2:5:2 (v/v/v). After centrifugation, the supernatants of chloroform phase were collected. For lipid derivatization, $500 \mu \mathrm{L}$ boron trifluoride-methanol solution was added to each sample after evaporating the 
chloroform and incubated at $90^{\circ} \mathrm{C}$ for $30 \mathrm{~min}(50 \mu \mathrm{L}$ saturated sodium chloride solution were added to stop the reaction immediately after the incubation). In this condition, lipid was mainly transesterified to fatty acid methyl ester and the calculated products reflected the total fatty acids from lipid in yeast cells. Later, $300 \mu \mathrm{L}$ hexane was added to extract fatty acids esters for GC-MS analysis.

Under the Shimadzu QP2010 Plus (Shimadzu, Kyoto, Japan) GC-MS system, $1 \mu 1$ samples were injected into the column (30 m-250 $\mu \mathrm{m}$ i.d. and $0.25 \mu \mathrm{m}$ thickness DB-5 capillary column) by splitless mode using an auto injector. Helium was used as carrier gas with the flow rate of $1.1 \mathrm{ml} / \mathrm{min}$. The injection and ion source temperatures were kept at 250 and $230{ }^{\circ} \mathrm{C}$, respectively. The oven temperature was set at $80{ }^{\circ} \mathrm{C}$ for $1 \mathrm{~min}$ and ramped to $250{ }^{\circ} \mathrm{C}$ by increased at $7{ }^{\circ} \mathrm{C} / \mathrm{min}$. The Shimadzu GC-MS solution software was used to acquire the chromatogram data and identify mass spectra (set from 35 to $600 \mathrm{~m} / \mathrm{z})$.

\subsubsection{HPLC analysis of compounds in media}

HPLC was performed to test the utilization of malonic acid. Samples from each supernatant were taken for HPLC analysis on a XDB-C18 column (Agilent, Santa Clara, USA). Compounds were separated with a solution of 50-mM potassium phosphate and acetonitrile with a ratio of 99:1 at $1.5 \mathrm{~mL} / \mathrm{min}, 25^{\circ} \mathrm{C}$. Malonic acids were detected by its UV absorbance at $210 \mathrm{~nm}$. All samples with three biological replicates were filtered through a $0.20 \mu \mathrm{m}$ filter before HPLC analysis. 


\subsection{Results}

\subsubsection{Growth rates of the wild-type strain and engineered strains}

The growth of each strain cultured in CM medium with malonic acids supplements was monitored by their OD 600 values. As shown in Figure 18, the growth curve of the engineered strains CEN-PAA-K, CEN-PAA-C, CEN-PAA-AB and CEN-PAA-ABC were slower than the control CEN-PAA-K (wide-type CEN.PK2-1C with blank plasmids), indicating the inhibition effect caused by the introduced pathways. Specifically, CEN-PAA-AB had the slowest growth during the first $24 \mathrm{~h}$, by displaying only $\sim 15 \%$ biomass increase of the wild type strain CEN-PAA-K. However, the density of all strains reached the similar level at stationary phase.

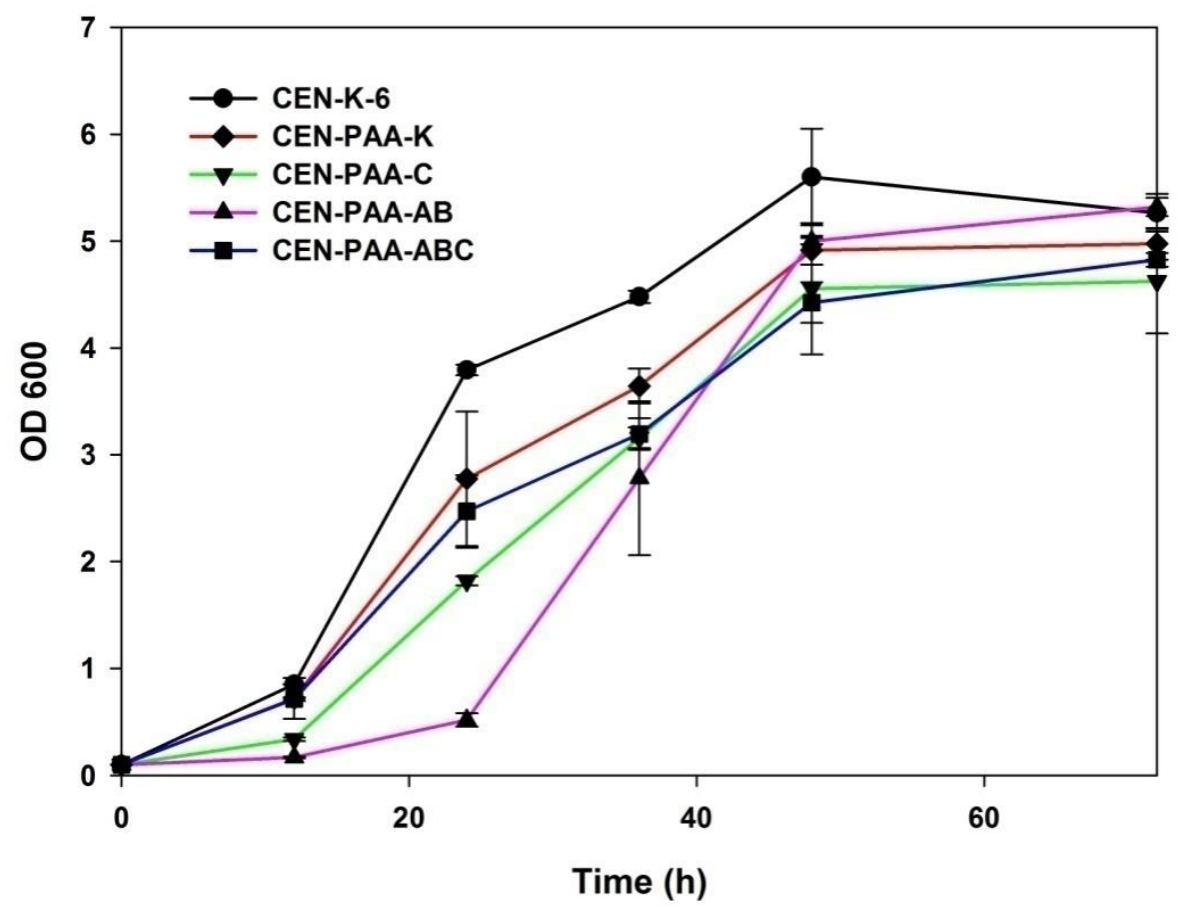

Figure 18. Growth profile of engineered strains in CM medium with malonic acids supply. Error bars represent the standard errors of three replicates. 


\subsubsection{Fatty acids production in strains with enhanced acetyl-CoA carboxylase} pathway

Pantothenate kinase (PanK) is considered to be the rate-limiting enzyme for CoA synthesis, which catalyzes the phosphorylation of pantothenate. However, PanK encoding gene $C A B 1$ in $S$. cerevisiae is reported to be transcribed at low level (Olzhausen, Schubbe et al. 2009). To enhance the acetyl-CoA carboxylase pathway, $C A B 1$ was overexpressed coupling with the PDH bypass, which include the overexpression of endogenous ALD6 (encoding acetaldehyde dehydrogenase) and introduction of SeAcs ${ }^{L 641 P}$ (encoding acetyl-CoA synthetase) from Salmonella enteric. The fatty acids contents were extracted from yeast cells after $24 \mathrm{~h}$ culturing and detected by GC-MS. The result from GC-MS showed that there were five types of fatty acids detected in yeast strains, i.e. tridecanoic acid (C14:0), hexadecanoic acid (C16:0), 9hexadecanoic acid (C16:1), octadecenoic acid (C18:0) and 9-octadecenoic acid (C18:1). No C16:2 or C18:2 fatty acids were detected in the strains of this study. As shown in Figure 19, the introduction of $\operatorname{Se} A c s^{L 641}$ and overexpression of $C A B 1$ and $A L D 6$ in yeast strain (CEN-PAA-K) led to a $\sim$-fold increase in the production of C16:0, C18:0, C18:1 after $24 \mathrm{~h}$ fermentation. On the other hand, previous study has demonstrated that $A C C 1^{S 659 A, S 1157 A}$ could increase the ACC activity by more than 3-fold (Shi, Chen et al. 2014). Therefore, when the mutated $A C C 1^{S 659 A, S 1157 A}$ was incorporated into CEN-PAA$\mathrm{K}$, the fatty acids production of the generated strain CEN-PAA-C has further improvement by showing about 3-fold increase in C16:0, 2-fold increase in C18:0, and 2-fold increase in C18:1 compared to the wild-type strain. 


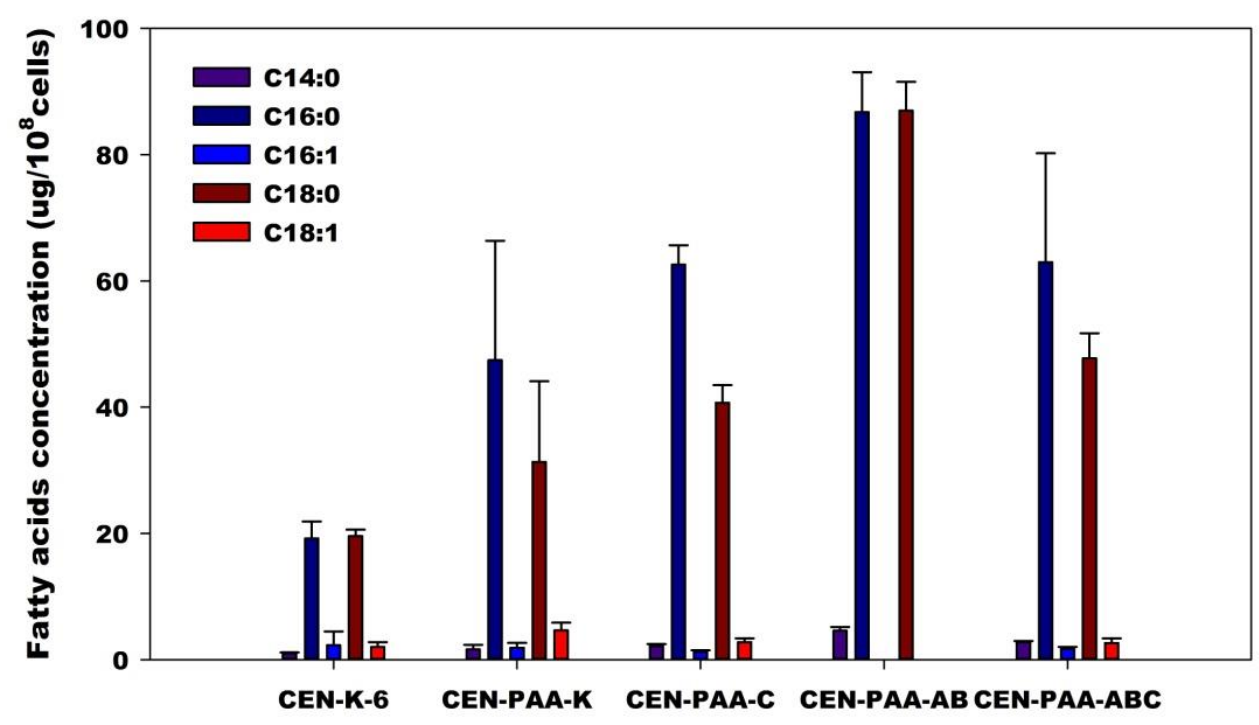

Figure 19. Fatty acids production of strains CEN-K-6, CEN-PAA-K, CEN-PAA-C, CEN-PAA-AB and CEN-PAA-ABC in CM medium with malonic acids supply after 24h. Error bars represent the standard errors of three replicates.

\subsubsection{Fatty acids production in strains with enhanced malonyl-CoA synthetase} pathway

In order to further elevate malonyl-CoA supply, malonic acid transporter MAE1 and malonyl-CoA synthetase $M a t B$ were successfully incorporated into CEN-PAA-K together to generate CEN-PAA-AB. From Figure 20, C16:0 and C18:0 of CEN-PAA$\mathrm{AB}$ reached $86.74 \mu \mathrm{g} / 10^{8}$ cells and $86.97 \mu \mathrm{g} / 10^{8}$ cells respectively, which was about 4fold that of the control CEN-K-6 (C16:0 19.22 $\mu \mathrm{g} / 10^{8}$ cells, C18:019.58 $\mu \mathrm{g} / 10^{8}$ cells). However, no C16:1 and C18:1 were monitored in CEN-PAA-AB. When integrate both acetyl-CoA pathway and malonyl-CoA synthetase pathway were integrated into CEN.PK2-1C, unexpectedly, the constructed strain CEN-PAA-ABC did not show 
further improved fatty acids production inside the cells. C14:0, C16:0, C16:1, C18:0 and C18:1 in CEN-PAA-ABC were about 2.78, 62.98, 1.6760, 47.72, $2.65 \mathrm{ug} / 10^{8}$ cells respectively after $24 \mathrm{~h}$ fermentation, which is about 3-fold of the control (Figure 20).

To test the incorporated malonyl-CoA synthetase pathway, MAE1 and MatB were transformed into CEN-K-6 separately to construct strain CEN-A and CEN-B in comparison of CEN-K-6, CEN-PAA-AB and CEN-PAA-ABC. Figure 20 shows that the amount of different fatty acids in CEN-A and CEN-B did not show much improvement than the control. However, when plasmid p41KAB containing both $M A E 1$ and $M a t B$ was transformed into the wild-type strain, the newly generated strain CEN-AB demonstrated improved fatty acids production by about 2-fold.

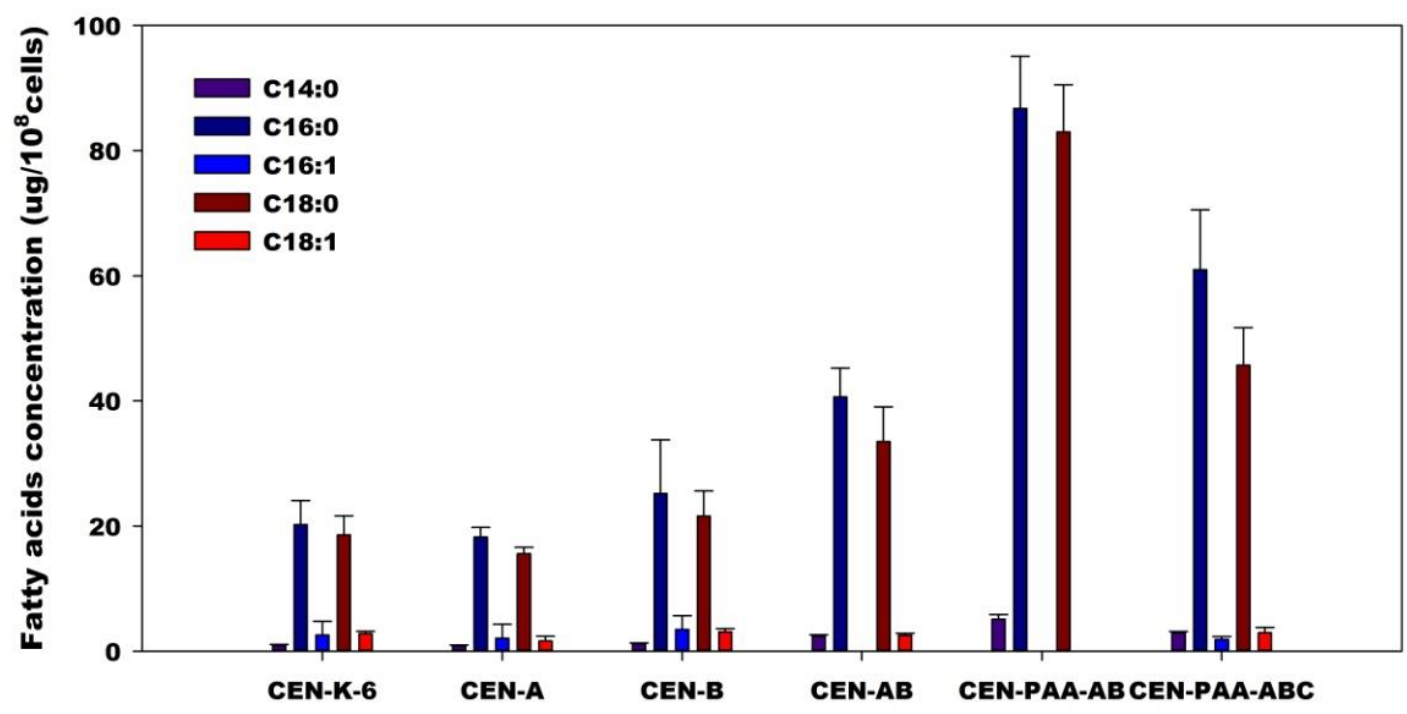

Figure 20. Fatty acids production of strains CEN-K-6, CEN-A, CEN-B, CEN-AB, CEN-PAA-AB and CEN-PAA-ABC in CM medium with malonic acids supply after 24h. Error bars represent the standard errors of three replicates. 


\subsubsection{Fatty acids production without malonic acids supply}

In order to test if the malonic acids supply for the malonyl-CoA synthetase pathway is the cause for the fatty acids production increase in CEN-PAA-AB, the intracellular fatty acids contents in the engineered strains were measured when cultured without malonic acids. In hypothesis, the fatty acids production in strain CEN-PAA-AB should decrease compared to its result when there are malonic acids supply. As displayed in Figure 21, CEN-K-6, CEN-PAA-K and CEN-PAA-C, CEN-PAA-ABC did not demonstrate much change in fatty acids production without malonic acids supply, but C14:0, C16:0 and C18:0 in CEN-PAA-AB all decreased greatly, which level was only about half of those in the case with malonic acids supplement. Interestingly, the fatty acids increase in CEN-PAA-ABC did not show much decline without malonic acids supplement.

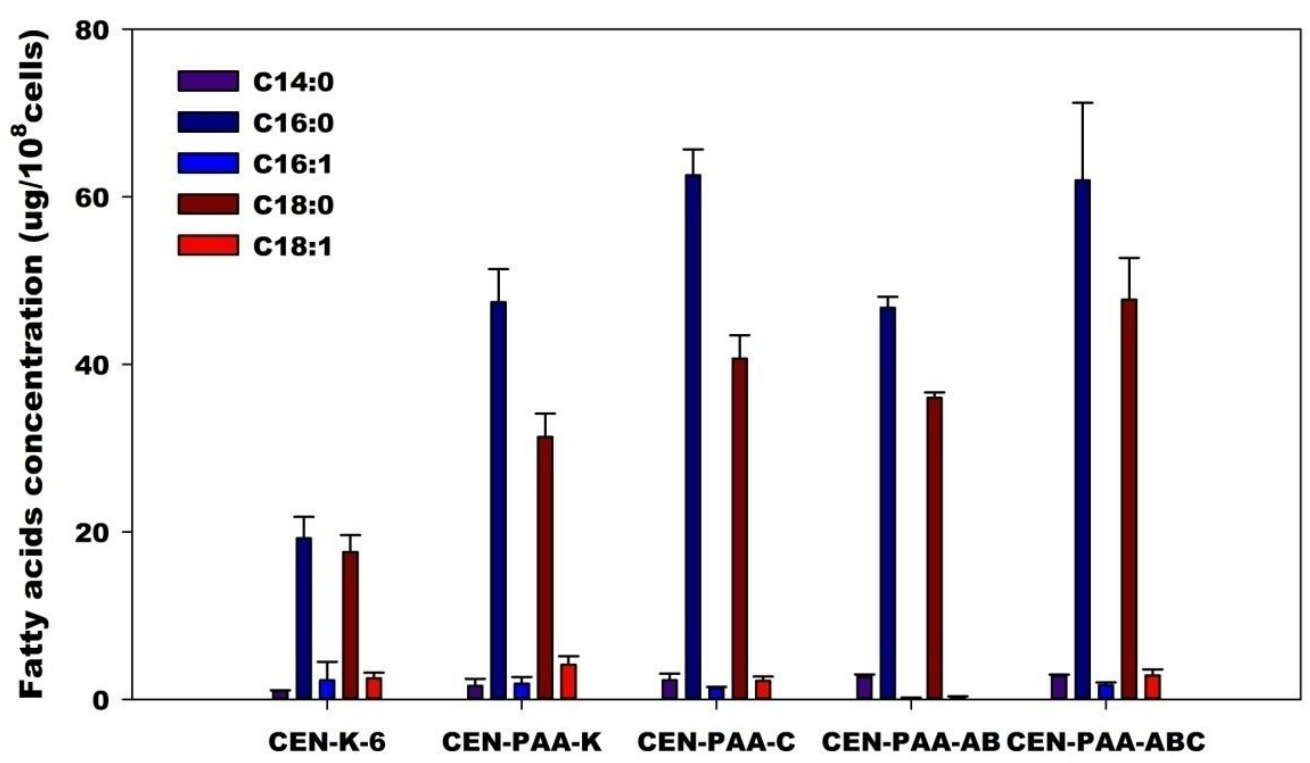

Figure 21. Fatty acids production of engineered strains CEN-K-6, CEN-PAA-K, CEN-PAA-C, CEN-PAA-AB and CEN-PAA-ABC in CM medium without malonic acids supply after 24h. Error 
bars represent the standard errors of three replicates.

\subsubsection{The utilization of malonic acids in engineered strains}

HPLC was performed to detect the consumption of malonic acid in the engineered strains. As shown in Figure 22, in CEN-PAA-AB and CEN-PAA-ABC, the concentration of malonic acids in the medium decreased from $0.35 \%$ (w/v) to 0.16 and $0.21 \%(\mathrm{w} / \mathrm{v})$ respectively during the fermentation process, but CEN-K-6, CEN-PAA-K and CEN-PAA-C demonstrated no apparent consumption of malonic acids. The results showed that only strains containing malonic acids transporter MAE1 could uptake exogenous malonate into their cytoplasm.

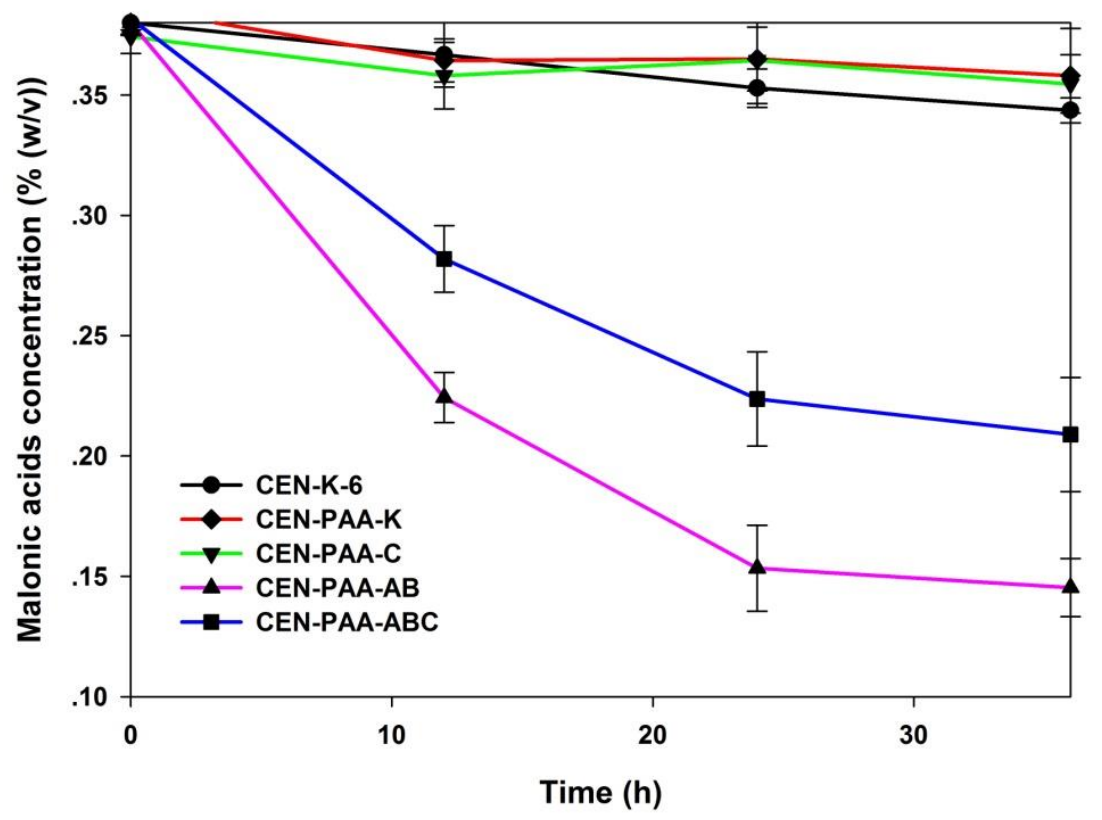

Figure 22. Malonic acids concentration in the medium of CEN-K-6, CEN-PAA-K, CEN-PAA-C,

CEN-PAA-AB and CEN-PAA-ABC during the culturing process. Error bars represent the standard errors of three replicates. 


\subsection{Discussion}

In yeast cell, limited supplies of cytosolic acetyl-CoA and malonyl-CoA are the major bottlenecks for de novo biosynthesis of fatty acids (van Roermund, Waterham et al. 2003). Therefore, coupled with our previous platform to direct the metabolic flux to the acetyl-CoA supply through overexpression of PanK, ALD6, SeAcs ${ }^{L 641 P}$ and $A C C 1^{S 659 A,}$ S1157A, we introduced a direct malonyl-CoA synthetase pathway by expressing malonyl-CoA synthetase (MatB) and dicarboxylic acid transporter (MAE1) to further increase malonyl-CoA synthesis for fatty acids accumulation. The fatty acids in yeast cells were qualified and the engineered strains demonstrated increase in fatty acids accumulation. Especially, the strain CEN-PAA-AB with both enhanced acytelCoA and malonyl-CoA pathways displayed $~ 5$-fold increase in C16:0 and C18:0.

From the growth curve, the engineered strains including CEN-PAA-K, CEN-PAAC, CEN-PAA-AB and CEN-PAA-ABC all demonstrated slow down growth than the control (CEN-K-6). Especially, CEN-PAA-AB showed much impaired growth during the first $24 \mathrm{~h}$. This phenomenon could be due to the low mono-unsaturated fatty acids composition in the engineered strains. From GC-MS result, among the five types of fatty acids detected in yeast strains, the mono-unsaturated fatty acids in CEN-PAA-K, CEN-PAA-C, and CEN-PAA-ABC, i.e. C16:1 and C18:1, consisted only 7.32\%, 3.64\% and $3.68 \%$ of the overall fatty acids respectively, much lower that of the control CENK-6 (9.66\%). Specifically, C16:1 and C18:1 were barely detected in CEN-PAA-AB cell. The unsaturated fatty acids composition in yeast cell consist almost exclusively of the mono-unsaturated fatty acids (Bossie and Martin 1989). Since mono-unsaturated 
fatty acids are essential for membrane expansion in growing cells (McDonough, Stukey et al. 1992), the decreased portion of $\mathrm{C} 16: 1$ and $\mathrm{C} 18: 1$ in the engineered strains may slower the synthesis of the membrane during the exponential phase, finally leading to their defective growths.

The fatty acid profile showed that there was approximate 2-fold increase in CENPAA-K with enhanced acetyl-CoA synthesis pathway. This result corresponds to our previous discoveries that overexpression of PanK, ALD6 and $\operatorname{Se} A c s^{L 641 P}$ increased acetyl-CoA level and naringenin concentration (a final model product of cytosolic acetyl-CoA) by 3 and 24.4-fold respectively (Wenshan, Bo et al. 2016). Pantothenate kinase (PanK) catalyzes the phosphorylation of pantothenate, which reaction considered to be the rate-limiting step for CoA synthesis (Olzhausen, Schubbe et al. 2009). Endogenous ALD6, and $\operatorname{SeAcs}{ }^{L 641 P}$ from Salmonella enteric have been successfully used in PDH bypass buildup for enhanced "acetyl-" content (Shiba, Paradise et al. 2007). The results from the fatty acids production in this study along with the previous experiment in naringenin production demonstrated that the expression cassette $C A B-A L D 6-\operatorname{Se} A c s^{L 641 P}$ is quite a valid platform for cytosolic acetyl-CoA accumulation. Additionally, within our expectation, introduction of a modified acetylCoA carboxylase $\left(A C C 1^{S 659 A, S 1157 A}\right)$ for more efficient conversion of acetyl-CoA to malonyl-CoA further improved fatty acids accumulation in cells. Nevertheless, when $A C C 1^{\text {S659A, S1157A }}$ was introduced into CEN-PAA-AB to combine with malonyl-CoA synthetase pathway, the fatty acids content in CEN-PAA-ABC did not show further improvement but an unexpected decrease. Since fatty acids biosynthesis (FAB) that 
occurs in fatty acid synthases (FASs) complex is under tight regulation, e.g. the feedback inhibition by acyl-CoAs or fatty acyl-ACPs (Knudsen, Valentin et al. 1999, Tehlivets, Scheuringer et al. 2007), the decreased fatty acids in CEN-PAA-ABC is possibly due to the negative feedback effect from the FAB pathway. However, when the medium is not supplied with malonic acids, the fatty acids increase in CEN-PAAABC did not show much decline. The incorporated ACC1 in CEN-PAA-ABC may play a role in transferring more acetyl-CoA into malonyl-CoA for more fatty acids production, compensating for the decrease caused by the absence of malonic acids supply.

The incorporation of direct malonyl-CoA synthetase pathway in CEN-PAA-K by expressing $M A E 1$ and MatB has further improved the fatty acids production when medium was supplied with malonic acids. The overall accumulated fatty acids production in CEN-PAA-AB reached $\sim 178.31 \mathrm{ug} / 10^{8}$ cells after $24 \mathrm{~h}$, which is comparable to the amount in previous reported engineered yeast strain $\left(145 \mathrm{ug} / 10^{8}\right.$ cells $)$ (Tang, Feng et al. 2013). The improvement in CEN-PAA-AB was lost when the medium does not contain malonic acids, suggesting that utilization of malonic acids is the cause for the improvement. This statement was further confirmed by detecting malonic acids concentration in the medium by HPLC, which showed that only the strains with expression cassette of MAE1-MatB displayed malonic acids consumption. The observation of rare fatty acids increase in strains with individual MAE1 and MatB (CEN-A and CEN-B) suggests that the malonyl-CoA synthesis pathway can be only functional when plasma membrane transporter and malonyl-CoA synthetase are 
combined. All these results agreed with the previous discovery that $S$. cerevisiae does not produce malonate for producing malonyl-CoA and cannot intake extracellular malonate (van Roermund, Waterham et al. 2003). The enhancement of fatty acids production $\mathrm{CEN}-\mathrm{AB}$ with $M A E 1-M a t B$ expression cassette further indicates that malonyl-CoA pathway constructed in this study was functional to elevate malonyl-CoA supply. 


\section{Chapter 6. Conclusion}

In this study, transcriptional engineering was used to improve ethanol tolerance and producion in $S$. cerevisiae. Rpb7 were used as targets for random mutagenesis via errorprone PCR. The bioalcohol tolerance mechanism, its relationship with production and recent engineering strategies were discussed in Chapter 4. In addition, through enhancing the precursor malonyl-CoA supply, fatty acids production in $S$. cerevisiae was improved.

The mutant (M1) isolated by random mutagenesis of Rpb7 showed much improved resistance towards ethanol. The ethanol titers of M1 was $\sim 122 \mathrm{~g} / \mathrm{L}(96.58 \%$ of the theoretical yield) under laboratory very high gravity (VHG) fermentation, $40 \%$ increase as compared to the control. Genome-wide DNA microarray assay showed that 369 genes had differential expression in M1 after $12 \mathrm{~h}$ VHG fermentation, which are involved in glycolysis, alcoholic fermentation, oxidative stress response, etc. As for improving fatty acids production in S. cerevisiae, the engineered strain (CEN-PAA-AB) with enhancement of acetyl-CoA carboxylase and malonyl-CoA synthetase pathways demonstrated about 5-fold improvement in fatty acids production after $24 \mathrm{~h}$ fermentation with the supply of $0.38 \%(\mathrm{w} / \mathrm{v})$ malonic acids. Based on these findings, we have the following conclusions:

1. Transcriptional engineering is an efficient alternative approach for strain engineering of performance improvement. Eukaryotic RNAP II enzyme could be an alternative gTME target in eliciting improved phenotype, which is probably also applicable to other eukaryotes. 
2. The increased ethanol production in M1 that has elevated expression of genes involved in ethanol production pathway implies that it is possible to engineer RNAP II to change the expression of multiple genes simultaneously to enhance the yield of desired products in the future.

3. The enhancement of fatty acids production of strain with MAE1-MatB expression cassette further indicates that malonyl-CoA pathway constructed was functional to elevate malonyl-CoA supply. The combination of malonyl-CoA pathway with the acetyl-CoA pathway could provide a valid platform for improving advanced biofuel production. 


\section{References}

Abe, H., Y. Fujita, Y. Takaoka, E. Kurita, S. Yano, N. Tanaka and K. Nakayama (2009). "Ethanol-tolerant

Saccharomyces cerevisiae strains isolated under selective conditions by over-expression of a proofreading-deficient DNA polymerase delta." J Biosci Bioeng 108(3): 199-204.

Alexandre, H., V. Ansanay-Galeote, S. Dequin and B. Blondin (2001). "Global gene expression during short-term ethanol stress in Saccharomyces cerevisiae." FEBS letters 498: 98-103.

Alexandre, H., L. Plourde, C. Charpentier and J. François (1998). "Lack of correlation between trehalose accumulation, cell viability and intracellular acidification as induced by various stresses in Saccharomyces cerevisiae." Microbiology 144: 1103-1111.

Alper, H., J. Moxley, E. Nevoigt, G. R. Fink and G. Stephanopoulos (2006). "Engineering yeast transcription machinery for improved ethanol tolerance and production." Science 314(5805): 1565-1568. Alper, H., J. Moxley, E. Nevoigt, G. R. Fink and G. Stephanopoulos (2006). "Engineering yeast transcription machinery for improved ethanol tolerance and production." Science 314(314): 1565-1568.

Alper, H. and G. Stephanopoulos (2007). "Global transcription machinery engineering: a new approach for improving cellular phenotype." Metab Eng 9(3): 258-267.

Anderson, M. J., S. L. Barker, C. Boone and V. Measday (2012). "Identification of RCN1 and RSA3 as ethanol-tolerant genes in Saccharomyces cerevisiae using a high copy barcoded library." FEMS Yeast Res 12(1): 48-60.

Ansanay-Galeote, V., B. Blondin, S. Dequin and J.-M. Sablayrolles (2001). "Stress effect of ethanol on fermentation kinetics by stationary-phase cells of Saccharomyces cerevisiae." Biotechnol lett 23: 677681. 
Armache, K. J., H. Kettenberger and P. Cramer (2003). "Architecture of initiation-competent 12-subunit RNA polymerase II." Proc Natl Acad Sci U S A 100(12): 6964-6968.

Atsumi, S., A. F. Cann, M. R. Connor, C. R. Shen, K. M. Smith, M. P. Brynildsen, K. J. Chou, T. Hanai and J. C. Liao (2008). "Metabolic engineering of Escherichia coli for 1-butanol production." Metab Eng 10(6): 305-311.

Atsumi, S., T. Y. Wu, I. M. Machado, W. C. Huang, P. Y. Chen, M. Pellegrini and J. C. Liao (2010).

"Evolution, genomic analysis, and reconstruction of isobutanol tolerance in Escherichia coli." Mol Syst

Biol 6: 449.

Avrahami-Moyal, L., S. Braun and D. Engelberg (2012). "Overexpression of PDE2 or SSD1-V in Saccharomyces cerevisiae W303-1A strain renders it ethanol-tolerant." FEMS Yeast Res 12(4): 447-455.

Bai, F. W., W. A. Anderson and M. Moo-Young (2008). "Ethanol fermentation technologies from sugar and starch feedstocks." Biotechnol Adv 26(1): 89-105.

Bai, F. W., L. J. Chen, Z. Zhang, W. A. Anderson and M. Moo-Young (2004). "Continuous ethanol production and evaluation of yeast cell lysis and viability loss under very high gravity medium conditions." J Biotechnol 110(3): 287-293.

Bailey, J. E. (1999). "Lessons from metabolic engineering for functional." Nat Biotechnol 17(7): 616618.

Bailey, J. E., A. Sburlati, V. Hatzimanikatis, K. Lee, W. A. Renner and P. S. Tsai (2002). "Inverse metabolic engineering: a strategy for directed genetic engineering of useful phenotypes." Biotechnol Bioeng 79(5): 568-579.

Bandara, A., S. Fraser, P. J. Chambers and G. A. Stanley (2009). "Trehalose promotes the survival of Saccharomyces cerevisiae during lethal ethanol stress, but does not influence growth under sublethal 
ethanol stress." FEMS Yeast Res 9(8): 1208-1216.

Basak, S. and R. Jiang (2012). "Enhancing E. coli tolerance towards oxidative stress via engineering its global regulator cAMP receptor protein (CRP)." PLoS One 7(12): e51179.

Basak, S., H. Song and R. Jiang (2012). "Error-prone PCR of global transcription factor cyclic AMP receptor protein for enhanced organic solvent (toluene) tolerance." Process Biochemistry 47(12): 21522158.

Benatuil, L., J. M. Perez, J. Belk and C. M. Hsieh (2010). "An improved yeast transformation method for the generation of very large human antibody libraries." Protein Eng Des Sel 23(4): 155-159.

Benjaphokee, S., D. Hasegawa, D. Yokota, T. Asvarak, C. Auesukaree, M. Sugiyama, Y. Kaneko, C. Boonchird and S. Harashima (2012). "Highly efficient bioethanol production by a Saccharomyces cerevisiae strain with multiple stress tolerance to high temperature, acid and ethanol." N Biotechnol 29(3): 379-386.

Biot-Pelletier, D. and V. J. Martin (2014). "Evolutionary engineering by genome shuffling." Appl Microbiol Biotechnol 98(9): 3877-3887.

Bossie, M. A. and C. E. Martin (1989). "Nutritional regulation of yeast delta-9 fatty acid desaturase activity." J Bacteriol 171(12): 6409-6413.

Bramucci, M. G., R. A. Larossa and D. R. Smulski (2013:US8455225). Yeast with increased butanol tolerance involving high osmolarity/glycerol response pathway. United States;.

Brat, D., C. Weber, W. Lorenzen, H. B. Bode and E. Boles (2012). "Cytosolic re-localization and optimization of valine synthesis and catabolism enables increased isobutanol production with the yeast Saccharomyces cerevisiae." Biotech for Biofuels 5(65).

C.Mackay, T. F. (2001). "Quantitative trait loci in Drosophila." Nature reviews genetics 2(1): 11-20. 
Cakar, Z. P., U. O. Seker, C. Tamerler, M. Sonderegger and U. Sauer (2005). "Evolutionary engineering of multiple-stress resistant Saccharomyces cerevisiae." FEMS Yeast Res 5(6-7): 569-578.

Cakar, Z. P., B. Turanli-Yildiz, C. Alkim and U. Yilmaz (2012). "Evolutionary engineering of Saccharomyces cerevisiae for improved industrially important properties." FEMS Yeast Res 12(2): 171182.

Cao, T. S., Z. Chi, G. L. Liu and Z. M. Chi (2014). "Expression of TPS1 gene from Saccharomycopsis fibuligera A11 in Saccharomyces sp. W0 enhances trehalose accumulation, ethanol tolerance, and ethanol production." Mol Biotechnol 56(1): 72-78.

Chandler, M., G. A. Stanley, P. Rogers and P. Chambers (2004). "A genomic approach to defining the ethanol stress response in the yeast." Annals of Microbiology 54(4): 427-454.

Chen, S. and Y. Xu (2014). "Adaptive Evolution of Saccharomyces cerevisiae with Enhanced Ethanol Tolerance for Chinese Rice Wine Fermentation." Appl Biochem Biotechnol 173(7): 1940-1954.

Chen, T., J. Wang, R. Yang, J. Li, M. Lin2 and Z. Lin (2011). "Laboratory-Evolved Mutants of an Exogenous Global Regulator, IrrE from Deinococcus radiodurans, Enhance Stress Tolerances of Escherichia coli." Plos One 6(1): 16228.

Chen, W. N. and K. Y. Tan (2013). "'Malonate uptake and metabolism in Saccharomyces cerevisiae"." Appl Biochem Biotechnol 171(1): 44-62.

Chen, X., K. F. Nielsen, I. Borodina, M. C. Kielland-Brandt and K. Karhumaa (2011). "Increased isobutanol production in Saccharomyces cerevisiae by overexpression of genes in valine metabolism." Biotech for Biofuels 4(21).

Chen, Y., L. Daviet, M. Schalk, V. Siewers and J. Nielsen (2013). "Establishing a platform cell factory through engineering of yeast acetyl-CoA metabolism." Metab Eng 15: 48-54. 
Chen, Y., L. Daviet, M. Schalk, V. Siewers and J. Nielsen (2013). "Establishing a platform cell factory through engineering of yeast acetyl-CoA metabolism." Metab Eng 15: 48-54.

Chi, K. O. (2007). "From Microbial Differentiation to Ribosome Engineering." Biosci Biotechnol Biochem 71(6): 1373-1386.

Choder, M. (2004). "Rpb4 and Rpb7: subunits of RNA polymerase II and beyond." Trends Biochem Sci 29(12): 674-681.

Choi, J. K. and Y. J. Kim (2008). "Epigenetic regulation and the variability of gene expression." Nat Genet 40(2): 141-147.

Chong, H., H. Geng, H. Zhang, H. Song, L. Huang and R. Jiang (2014). "Enhancing E. coli isobutanol tolerance through engineering its global transcription factor cAMP receptor protein (CRP)." Biotechnol Bioeng 111(4): 700-708.

Chong, H., L. Huang2, J. Yeow, I. Wang, H. Zhang, H. Song and R. Jiang (2013). "Improving Ethanol Tolerance of Escherichia coli by Rewiring Its Global Regulator cAMP Receptor Protein (CRP)." Plos One 8: e57628.

Connor, M. R., A. F. Cann and J. C. Liao (2010). "3-Methyl-1-butanol production in Escherichia coli: random mutagenesis and two-phase fermentation." Appl Microbiol Biotechnol 86(4): 1155-1164.

Costa, V., M. A. Amorim, E. Reis, A. Quintanilha and P. Moradas-Ferreira (1997). "Mitochondria1 superoxide disrnutase is essential for ethanol tolerance of Saccharomyces cerevisiae in the post-diauxic phase." MicrObiology 143: 1649-1656.

Cramer, P. (2000). "Architecture of RNA Polymerase II and Implications for the Transcription Mechanism." Science 288(5466): 640-649.

Cramer, P. (2004). "RNA polymerase II structure: from core to functional complexes." Curr Opin Genet 
Dev 14(2): 218-226.

Demasi, A. P., G. A. Pereira and L. E. Netto (2006). "Yeast oxidative stress response. Influences of cytosolic thioredoxin peroxidase I and of the mitochondrial functional state." FEBS J 273(4): 805-816. Demirbas and Ayhan (2008). "Biofuels sources, biofuel policy, biofuel economy and global biofuel projections." Energy Convers. Manage. 49(8): 2106-2116.

Dinh, T. N., K. Nagahisa, T. Hirasawa, C. Furusawa and H. Shimizu (2008). "Adaptation of Saccharomyces cerevisiae cells to high ethanol concentration and changes in fatty acid composition of membrane and cell size." PLoS One 3(7): e2623.

Du, X. and H. Takagi (2007). "N-Acetyltransferase Mpr1 confers ethanol tolerance on Saccharomyces cerevisiae by reducing reactive oxygen species." Appl Microbiol Biotechnol 75(6): 1343-1351.

Duitama, J., A. Sánchez-Rodríguez, A. Goovaerts, S. Pulido-Tamayo, G. Hubmann, M. R. FoulquiéMoreno, J. M. Thevelein, K. J. Verstrepen and K. Marchal (2014). "Improved linkage analysis of Quantitative Trait Loci using bulk segregants unveils a novel determinant of high ethanol tolerance in yeast." BMC Genomics 15.

Espinazo-Romeu, M., J. M. Cantoral, E. Matallana and A. Aranda (2008). "Btn2p is involved in ethanol tolerance and biofilm formation in flor yeast." FEMS Yeast Res 8(7): 1127-1136.

Ezeji, T. C., N. Qureshi and H. P. Blaschek (2007). "Bioproduction of butanol from biomass: from genes to bioreactors." Curr Opin Biotechnol 18(3): 220-227.

F.W.Baia, W.A.Andersona and M.Moo-Young (2008). "Ethanol fermentation technologies from sugar and starch feedstocks." Biotechnol Adv 26(1): 89-105.

Feller, S. E., C. A. Brown, D. T. Nizza and K. Gawrisch (2002). "Nuclear Overhauser enhancement spectroscopy cross-relaxation rates and ethanol distribu- tion across membranes." Biophysical Journal 
82(3): 1396-1404.

Feng, X., J. Lian and H. Zhao (2015). "Metabolic engineering of Saccharomyces cerevisiae to improve 1-hexadecanol production." Metab Eng 27(10-19).

Fischer, C. R., D. Klein-Marcuschamer and G. Stephanopoulos (2008). "Selection and optimization of microbial hosts for biofuels production." Metab Eng 10(6): 295-304.

Flikweert, M. t., V. D. Z. L, J. WM, S. HY, V. D. JP and P. JT (1996). "Pyruvate decarboxylase: an indispensable enzyme for growth of Saccharomyces cerevisiae on glucose." Yeast 12(3): 247-257.

Fujita, K., A. Matsuyama, Y. Kobayashi and H. Iwahashi (2006). "The genome-wide screening of yeast deletion mutants to identify the genes required for tolerance to ethanol and other alcohols." FEMS Yeast Res 6(5): 744-750.

Furukawa, K., H. Obata, H. Kitano, H. Mizoguchi and S. Hara (2004). "Effect of cellular inositol content on ethanol tolerance of Saccharomyces cerevisiae in sake brewing." Journal of Bioscience and Bioengineering 98(2): 107-113.

Ghiaci, P., J. Norbeck and C. Larsson (2013). "Physiological adaptations of Saccharomyces cerevisiae evolved for improved butanol tolerance." Biotechnol Biofuels 6(1): 101.

Gibson, B. R., S. J. Lawrence, J. P. Leclaire, C. D. Powell and K. A. Smart (2007). "Yeast responses to stresses associated with industrial brewery handling." FEMS Microbiol Rev 31(5): 535-569.

Grunstein, M. (1997). "Histone acetylation in chromatin structure and transcription." Nature 389(6649): 349-352.

Guo, W., J. Sheng, H. Zhao and X. Feng (2016). "Metabolic engineering of Saccharomyces cerevisiae to produce 1-hexadecanol from xylose." Microb Cell Fact 15(24).

Gutiérrez-Lomelí, M., J. C. Torres-Guzmán, G. A. González-Hernández, L. A. Cira-Chávez, C. Pelayo- 
Ortiz and J. d. J. Ramírez-Córdova (2008). "Antonie Van Leeuwenhoek." Overexpression of ADH1 and HXT1 genes in the yeast Saccharomyces cerevisiae improves the fermentative efficiency during tequila elaboration. 93(4): 363-371.

Gygi, S. P., Y. Rochon, B. R. Franza and R. Aebersold (1999). "Correlation between Protein and mRNA Abundance in Yeast." Molecular and Cellular Biology 19(3): 1720-1730.

Harel-Sharvit, L., N. Eldad, G. Haimovich, O. Barkai, L. Duek and M. Choder (2010). "RNA polymerase II subunits link transcription and mRNA decay to translation." Cell 143(4): 552-563.

Henderson, C. M. and D. E. Block (2014). "Examining the role of membrane lipid composition in determining the ethanol tolerance of Saccharomyces cerevisiae." Appl Environ Microbiol 80(10): 29662972.

Henderson, C. M., M. Lozada-Contreras, V. Jiranek, M. L. Longo and D. E. Block (2013). "Ethanol production and maximum cell growth are highly correlated with membrane lipid composition during fermentation as determined by lipidomic analysis of 22 Saccharomyces cerevisiae strains." Appl Environ Microbiol 79(1): 91-104.

Hersh, M. N., R. G. Ponder, P. J. Hastings and S. M. Rosenberg (2004). "Adaptive mutation and amplification in Escherichia coli: two pathways of genome adaptation under stress." Res Microbiol 155(5): 352-359.

Hill, J., E. Nelson, D. Tilman, S. Polasky and D. Tiffany (2006). "Environmental, economic, and energetic costs and benefits of biodiesel and ethanol biofuels." Proc Natl Acad Sci U S A 103(30): 1120611210.

Hirasawa, T., K. Yoshikawa, Y. Nakakura, K. Nagahisa, C. Furusawa, Y. Katakura, H. Shimizu and S. Shioya (2007). "Identification of target genes conferring ethanol stress tolerance to Saccharomyces 
cerevisiae based on DNA microarray data analysis." J Biotechnol 131(1): 34-44.

Ho, W.-C. and J. Zhang (2014). "The genotype-phenotype map of yeast complex traits: basic parameters and the role of natural selection." Mol Biol Evol 31(6): 1568-1580.

Hong, K. K. and J. Nielsen (2012). "Metabolic engineering of Saccharomyces cerevisiae: a key cell factory platform for future biorefineries." Cell Mol Life Sci 69(16): 2671-2690.

Hong, M. E., K. S. Lee, B. J. Yu, Y. J. Sung, S. M. Park, H. M. Koo, D. H. Kweon, J. C. Park and Y. S. Jin (2010). "Identification of gene targets eliciting improved alcohol tolerance in Saccharomyces cerevisiae through inverse metabolic engineering." J Biotechnol 149(1-2): 52-59.

Hong, S. H., M. Hegde, J. Kim, X. Wang, A. Jayaraman and T. K. Wood (2012). "Synthetic quorumsensing circuit to control consortial biofilm formation and dispersal in a microfluidic device." Nat Commun 3: 613 .

Hong, S. H., X. Wang and T. K. Wood (2010). "Controlling biofilm formation, prophage excision and cell death by rewiring global regulator H-NS of Escherichia coli." Microb Biotechnol 3(3): 344-356.

Honga, M.-E., K.-S. Leea, B. J. Yub, Y.-J. Sunga, S. M. Parkb, H. M. Koob, D.-H. Kweona, J. C. Parkb and Y.-S. Jin (2010). "Identification of gene targets eliciting improved alcohol tolerance in Saccharomyces cerevisiae through inverse metabolic engineering." J Biotechnol 149(1-2): 52-59.

Hou, J., N. F. Lages, M. Oldiges and G. N. Vemuri (2009). "Metabolic impact of redox cofactor perturbations in Saccharomyces cerevisiae." Metab Eng 11(4-5): 253-261.

Hou, L. (2010). "Improved production of ethanol by novel genome shuffling in Saccharomyces cerevisiae." Appl Biochem Biotechnol 160(4): 1084-1093.

Hu, X. H., M. H. Wang, T. Tan, J. R. Li, H. Yang, L. Leach, R. M. Zhang and Z. W. Luo (2007). "Genetic dissection of ethanol tolerance in the budding yeast Saccharomyces cerevisiae." Genetics 175(3): 1479- 
1487.

Izawa, S., Y. Inoue and A. Kimura (1996). "Importance of catalase in the adaptive response to hydrogen peroxide." Biochem J 15(320): 61-67.

Izawa, S., T. Kita, K. Ikeda and Y. Inoue (2008). "Heat shock and ethanol stress provoke distinctly different responses in 3'-processing and nuclear export of HSP mRNA in Saccharomyces cerevisiae." Biochem J 414(1): 111-119.

Jasiak, A. J., H. Hartmann, E. Karakasili, M. Kalocsay, A. Flatley, E. Kremmer, K. Strasser, D. E. Martin, J. Soding and P. Cramer (2008). "Genome-associated RNA polymerase II includes the dissociable Rpb4/7 subcomplex." J Biol Chem 283(39): 26423-26427.

Jiménez, J. and T. Benítez (1988). "Selection of Ethanol-Tolerant Yeast Hybrids in pH-Regulated Continuous Culture." Appl Environ Microbiol 54(4): 917-922.

Jong, B. d., V. Siewers and J. Nielsen (2012). "Systems biology of yeast: enabling technology for development of cell factories for production of advanced biofuels." Curr Opin Biotechnol 23(4): 624630.

Jung, Y. J. and H. D. Park (2005). "Antisense-mediated inhibition of acid trehalase (ATH1) gene expression promotes ethanol fermentation and tolerance in Saccharomyces cerevisiae." Biotechnol Lett 27(23-24): 1855-1859.

Kama, R., M. Robinson and J. E. Gerst (2007). "Btn2, a Hook1 ortholog and potential Batten diseaserelated protein, mediates late endosome-Golgi protein sorting in yeast." Mol Cell Biol 27(2): 605-621. Kasavi, C., S. Eraslan, K. Y. Arga, E. T. Oner and B. Kirdar (2014). "A system based network approach to ethanol tolerance in Saccharomyces cerevisiae." BMC Syst Biol 8(90).

Kildegaard, K. R., N. B. Jensen, K. Schneider, E. Czarnotta, E. Ozdemir, T. Klein, J. Maury, B. E. Ebert, 
H. B. Christensen, Y. Chen, I. K. Kim, M. J. Herrgard, L. M. Blank, J. Forster, J. Nielsen and I. Borodina

(2016). "Engineering and systems-level analysis of Saccharomyces cerevisiae for production of 3hydroxypropionic acid via malonyl-CoA reductase-dependent pathway." Microb Cell Fact 15: 53.

Kim, H. S., N. R. Kim and W. Choi (2011). "Total fatty acid content of the plasma membrane of Saccharomyces cerevisiae is more responsible for ethanol tolerance than the degree of unsaturation." Biotechnol Lett 33(3): 509-515.

Kim, I.-S., Y.-S. Kim and H.-S. Yoon (2013). "Expression of salt-induced 2-Cys peroxiredoxin from Oryza sativa increases stress tolerance and fermentation capacity in genetically engineered yeast Saccharomyces cerevisiae." Appl Microbiol Biotechnol 97(8): 3519-3533.

Kim, I.-S., S.-Y. Shin, S.-H. Kim and H.-S. Yoon (2012). "Ectopic expression of sweet potato MuS1 increases acquired stress tolerance and fermentation yield in Saccharomyces cerevisiae." J Microbiol 50(3): 544-546.

Kim, J., P. Alizadeh, T. Harding, A. Hefner-Gravink and D. J. Klionsky (1996). "Disruption of the Yeast ATH1 Gene Confers Better Survival after Dehydration, Freezing, and Ethanol Shock: Potential Commercial Applications." Appl Environ Microbiol 62(5): 1563-1569.

Kim, N. R., J. Yang, H. Kwon, J. An, W. Choi and W. Kim (2013). "Mutations of the TATA-binding protein confer enhanced tolerance to hyperosmotic stress in Saccharomyces cerevisiae." Appl Microbiol Biotechnol 97(18): 8227-8238.

Kim, Y. S. (2002). "Malonate Metabolism Biochemistry, Molecular Biology, Physiology." J Biochem Mol Biol 30(35): 443-451.

Klein-Marcuschamer, D., C. N. Santos, H. Yu and G. Stephanopoulos (2009). "Mutagenesis of the bacterial RNA polymerase alpha subunit for improvement of complex phenotypes." Appl Environ 
Microbiol 75(9): 2705-2711.

Klein-Marcuschamer, D. and G. Stephanopoulos (2008). "Assessing the potential of mutational strategies to elicit new phenotypes in industrial strains." Proc Natl Acad Sci U S A 105(7): 2319-2324.

Knudsen, J., M. Valentin, J. J. F\sgmaelig;rgeman, T. B. F. Neergaard and B. Gaigg (1999). "Role of acylCoA binding protein in acyl-CoA metabolism and acyl-CoA-mediated cell signaling." Mol Cell Biochem 192: $95-103$.

Koleske, A. J. and R. A. Young (1994). "An RNA polymerase II holoenzyme responsive to activators." Nature 368(6470): 466 - 469.

Kubota, S., I. Takeo, K. Kume, M. Kanai, A. Shitamukai, M. Mizunuma, T. Miyakawa, H. Shimoi, H. Iefuji and D. Hirata (2004). "Effect of ethanol on cell growth of budding yeast: genes that are important for cell growth in the presence of ethanol." Biosci Biotechnol Biochem 68(4): 968-972.

Kurosawa, K., T. Hosaka, N. Tamehiro, T. Inaoka and K. Ochi (2006). "Improvement of alpha-amylase production by modulation of ribosomal component protein S12 in Bacillus subtilis 168." Appl Environ Microbiol 72(1): 71-77.

Kyung-Mi Yang, Ji-Min Woo, Sun-Mee Lee and J.-B. Park (2013). "Improving ethanol tolerance of Saccharomyces cerevisiae by overexpressing an ATP-binding cassette efflux pump." Chemical Engineering Science 103(15): 74-78.

Lam, F. H., A. Ghaderi, G. R. Fink and G. Stephanopoulos (2014). "Biofuels. Engineering alcohol tolerance in yeast." Science 346(6205): 71-75.

Lewis, J. A., I. M. Elkon, M. A. McGee, A. J. Higbee and A. P. Gasch (2010). "Exploiting natural variation in Saccharomyces cerevisiae to identify genes for increased ethanol resistance." Genetics 186(4): 1197-1205. 
Li, B. Z., J. S. Cheng, M. Z. Ding and Y. J. Yuan (2010). "Transcriptome analysis of differential responses of diploid and haploid yeast to ethanol stress." J Biotechnol 148(4): 194-203.

Li, X., D. Guo, Y. Cheng, F. Zhu, Z. Deng and T. Liu (2014). "Overproduction of fatty acids in engineered Saccharomyces cerevisiae." Biotechnol Bioeng 111(9): 1841-1852.

Lian, J., T. Si, N. U. Nair and H. Zhao (2014). "Design and construction of acetyl-CoA overproducing Saccharomyces cerevisiae strains." Metab Eng 24: 139-149.

Lian, J. and H. Zhao (2015). "Recent advances in biosynthesis of fatty acids derived products in Saccharomyces cerevisiae via enhanced supply of precursor metabolites." J Ind Microbiol Biotechnol 42(3): 437-451.

Liang, Q., Q. Wang, C. Gao, Z. Wang and Q. Qi (2011). "The effect of cyclodextrins on the ethanol tolerance of microorganisms suggests potential application." J Ind Microbiol Biotechnol 38(6): 753-756. Lin, Y. and S. Tanaka (2006). "Ethanol fermentation from biomass resources: current state and prospects." Appl Microbiol Biotechnol 69(6): 627-642.

Lin, Z., Y. Zhang and J. Wang (2013). "Engineering of transcriptional regulators enhances microbial stress tolerance." Biotechnol Adv 31(6): 986-991.

Listman, J. B., R. T. Malison, K. Sanichwankul, C. Ittiwut, A. Mutirangura and J. Gelernter (2011). "Southeast Asian origins of five Hill Tribe populations and correlation of genetic to linguistic relationships inferred with genome-wide SNP data." Am J Phys Anthropol 144(2): 300-308.

Liu, C.-G., N. Wang, Y.-H. Lin and F.-W. Bai (2012). "Very high gravity ethanol fermentation by flocculating yeast under redox potential-controlled conditions." Biotechnology for Biofuels 5(61).

Liu, H., K. Liu, M. Yan, L. Xu and P. Ouyang (2011). "gTME for improved adaptation of Saccharomyces cerevisiae to corn cob acid hydrolysate." Appl Biochem Biotechnol 164(7): 1150-1159. 
Liu, J. J., W. T. Ding, G. C. Zhang and J. Y. Wang (2011). "Improving ethanol fermentation performance of Saccharomyces cerevisiae in very high-gravity fermentation through chemical mutagenesis and meiotic recombination." Appl Microbiol Biotechnol 91(4): 1239-1246.

Liu, W., B. Zhang and R. Jiang (2017). "Improving acetyl-CoA biosynthesis in Saccharomyces cerevisiae via the overexpression of pantothenate kinase and PDH bypass." Biotechnol Biofuels 17(0): 41.

Liu, Z., X. Zhao and F. Bai (2013). "Production of xylanase by an alkaline-tolerant marine-derived Streptomyces viridochromogenes strain and improvement by ribosome engineering." Appl Microbiol Biotechnol 97(10): 4361-4368.

Lotan, R., V. Goler-Baron, L. Duek, G. Haimovich and M. Choder (2007). "The Rpb7p subunit of yeast RNA polymerase II plays roles in the two major cytoplasmic mRNA decay mechanisms." J Cell Biol 178(7): 1133-1143.

Luhe, A. L., L. Tan, J. Wu and H. Zhao (2011). "Increase of ethanol tolerance of Saccharomyces cerevisiae by error-prone whole genome amplification." Biotechnol Lett 33(5): 1007-1011.

Lv, X. A., Y. Y. Jin, Y. D. Li, H. Zhang and X. L. Liang (2013). "Genome shuffling of Streptomyces viridochromogenes for improved production of avilamycin." Appl Microbiol Biotechnol 97(2): 641-648. Ma, M. and L. Z. Liu (2010). "Quantitative transcription dynamic analysis reveals candidate genes and key regulators for ethanol tolerance in Saccharomyces cerevisiae." BMC Microbiol 10: 169.

Ma, M. and Z. L. Liu (2010). "Mechanisms of ethanol tolerance in Saccharomyces cerevisiae." Appl Microbiol Biotechnol 87(3): 829-845.

Manrubia, S. C., C. Escarmis, E. Domingo and E. Lazaro (2005). "High mutation rates, bottlenecks, and robustness of RNA viral quasispecies." Gene 347(2): 273-282.

Mansure, J. J. C., A. D. Panek, L. M. Crowe and J. H. Crowe (1994). "Trehalose inhibits ethanol effects 
on intact yeast-cells and liposomes." Biochim Biophys Acta 1191: 309-316.

Matsuda, T., S. Fujimura, H. Suda, Y. Matsufuji and J. Nakagawa (2011). "Alteration of ethanol tolerance caused by the deficiency in the genes associated with histone deacetylase complex in budding yeast." Biosci Biotechnol Biochem 75(9): 1829-1831.

Mauricio, J., E. Valero, C. Millan and J. Ortega (2001). "Changes in nitrogen compounds in must and wine during fermentation and biological aging by flor yeasts." J. Agric. Food Chem 49(7): 3310-3315.

McDonough, V. M., J. E. Stukey and C. E. Martin (1992). "Specificity of unsaturated fatty acid-regulated expression of the Saccharomyces cerevisiae OLE1 gene." J Biol Chem 267(9): 5931-5936.

Meka, H., F. Werner, S. C. Cordell, S. Onesti and P. Brick (2005). "Crystal structure and RNA binding of the Rpb4/Rpb7 subunits of human RNA polymerase II." Nucleic Acids Res 33(19): 6435-6444.

Mizoguchi, H. and S. Hara (1998). "Permeability barrier of the yeast plasma membrane induced by ethanol." Journal of Fermentation and Bioengineering 86(1): 25-29.

Moon, M. H., J. Ryu, Y.-H. Choeng, S.-K. Hong, H. A. Kang and Y. K. Chang (2012). "Enhancement of stress tolerance and ethanol production in Saccharomyces cerevisiae by heterologous expression of a trehalose biosynthetic gene from Streptomyces albus." Biotechnology and Bioprocess Engineering 17(5): 986-996.

Morales, L. and B. Dujon (2012). "Evolutionary role of interspecies hybridization and genetic exchanges in yeasts." Microbiol Mol Biol Rev 76(4): 721-739.

Na, D., S. M. Yoo, H. Chung, H. Park, J. H. Park and S. Y. Lee (2013). "Metabolic engineering of Escherichia coli using synthetic small regulatory RNAs." Nat Biotechnol 31(2): 170-174.

Naik, S. N., V. V. Goud, P. K. Rout and A. K. Dalai (2010). "Production of first and second generation biofuels: A comprehensive review." Renewable and Sustainable Energy Reviews 14(2): 578-597. 
Nielsen, J. and M. C. Jewett (2008). "Impact of systems biology on metabolic engineering of Saccharomyces cerevisiae." FEMS Yeast Res 8(1): 122-131.

Nielsen, J. and J. D. Keasling (2016). "Engineering Cellular Metabolism." Cell 164(6): 1185-1197.

Nijkamp, J. F., M. van den Broek, E. Datema, S. de Kok, L. Bosman, M. A. Luttik, P. Daran-Lapujade, W. Vongsangnak, J. Nielsen, W. H. Heijne, P. Klaassen, C. J. Paddon, D. Platt, P. Kotter, R. C. van Ham, M. J. Reinders, J. T. Pronk, D. de Ridder and J. M. Daran (2012). "De novo sequencing, assembly and analysis of the genome of the laboratory strain Saccharomyces cerevisiae CEN.PK113-7D, a model for modern industrial biotechnology." Microb Cell Fact 11: 36.

Ochi, K. (2007). "From microbial differentiation to ribosome engineering." Biosci Biotechnol Biochem 71(6): 1373-1386.

Olano, C., F. Lombo, C. Mendez and J. A. Salas (2008). "Improving production of bioactive secondary metabolites in actinomycetes by metabolic engineering." Metab Eng 10(5): 281-292.

Olzhausen, J., S. Schubbe and H. J. Schuller (2009). "Genetic analysis of coenzyme A biosynthesis in the yeast Saccharomyces cerevisiae: identification of a conditional mutation in the pantothenate kinase gene CAB1." Curr Genet 55(2): 163-173.

Özcan, S. and M. Johnston (1999). "Function and Regulation of Yeast Hexose Transporters." Microbiol Mol Biol Rev 63(3): 554-569.

Pais, T. M., M. R. Foulquie-Moreno, G. Hubmann, J. Duitama, S. Swinnen, A. Goovaerts, Y. Yang, F. Dumortier and J. M. Thevelein (2013). "Comparative polygenic analysis of maximal ethanol accumulation capacity and tolerance to high ethanol levels of cell proliferation in yeast." PLoS Genet 9(6): e1003548.

Paradise, E. M., J. Kirby, R. Chan and J. D. Keasling (2008). "Redirection of flux through the FPP branch- 
point in Saccharomyces cerevisiae by down-regulating squalene synthase." Biotechnol Bioeng 100(2): $371-378$

Park, J. H. and S. Y. Lee (2008). "Towards systems metabolic engineering of microorganisms for amino acid production." Curr Opin Biotechnol 19(5): 454-460.

Park, K. S., D. K. Lee, H. Lee, Y. Lee, Y. S. Jang, Y. H. Kim, H. Y. Yang, S. I. Lee, W. Seol and J. S. Kim (2003). "Phenotypic alteration of eukaryotic cells using randomized libraries of artificial transcription factors." Nat Biotechnol 21(10): 1208-1214.

Patnaik, R. (2008). "Engineering Complex Phenotypes in Industrial Strains." Biotechnology Progress 24(1): $38-47$.

Patnaik, R., S. Louie, V. Gavrilovic, K. Perry, W. P. C. Stemmer, C. M. Ryan and S. d. Cardayré (2002). "Genome shuffling of Lactobacillus for improved acid tolerance." Nature Biotechnology 20(707-712).

Peralta-Yahya, P. P., M. Ouellet, R. Chan, A. Mukhopadhyay, J. D. Keasling and T. S. Lee (2011). "Identification and microbial production of a terpene-based advanced biofuel." Nat Commun 2: 483.

Petri, R. and C. Schmidt-Dannert (2004). "Dealing with complexity: evolutionary engineering and genome shuffling." Curr Opin Biotechnol 15(4): 298-304.

Pinel, D., F. D'Aoust, S. B. d. Cardayre, P. K. Bajwa, H. Lee and V. J. J. Martin (2011). "Saccharomyces cerevisiae genome shuffling through recursive population mating leads to improved tolerance to spent sulfite liquor." Appl Environ Microbiol 77(14): 4736-4743.

Pronk, J. T., H. Y. Steensmays and J. P. V. Dijken (1996). "Pyruvate metabolism in Saccharomyces cerevisiae." Yeast 12(16): 1607-1633.

Puligundla, P., D. Smogrovicova, V. S. Obulam and S. Ko (2011). "Very high gravity (VHG) ethanolic brewing and fermentation: a research update." J Ind Microbiol Biotechnol 38(9): 1133-1144. 
Qi, L. S., M. H. Larson, L. A. Gilbert, J. A. Doudna, J. S. Weissman, A. P. Arkin and W. A. Lim (2013).

"Repurposing CRISPR as an RNA-guided platform for sequence-specific control of gene expression."

Cell 152(5): 1173-1183.

Raju, E. and G. Divakar (2013). "Bacillus Cereus GD 55 Strain Improvement by Physical and Chemical Mutagenesis for Enhanced Production of Fibrinolytic Protease." International Journal of Pharma Sciences and Research 4: 81-93.

Remize, F., E. Andrieu and S. Dequin (2000). "Engineering of the pyruvate dehydrogenase bypass in Saccharomyces cerevisiae: role of the cytosolic $\operatorname{Mg}(2+)$ and mitochondrial $\mathrm{K}(+)$ acetaldehyde dehydrogenases Ald6p and Ald4p in acetate formation during alcoholic fermentation." Appl Environ Microbiol 66(8): 3151-3159.

Rock, C. O., R. B. Calder, M. A. Karim and S. Jackowski (2000). "Pantothenate kinase regulation of the intracellular concentration of coenzyme A." J Biol Chem 275(2): 1377-1383.

Romphruk, A. V., A. Oka, A. Romphruk, M. Tomizawa, C. Choonhakarn, T. K. Naruse, C. Puapairoj, G. Tamiya, C. Leelayuwat and H. Inoko (2003). "Corneodesmosin gene no evidence for PSORS 1 gene in North-eastern Thai psoriasis patients." Tissue Antigens 62(3): 217-224.

Rosa, M. F. and I. Sś-Correia (1996). "Intracellular acidification does not account for inhibition of Saccharomyces cerevisiae growth in the presence of ethanol." FEMS Microbiol Lett 135(2-3): 271-274. Rosillo-Calle, F. (2006). "Global market for bioethanol historical trends and future prospects." Energy for Sustainable Development $\mathbf{X}(1)$.

Runguphan, W. and J. D. Keasling (2014). "Metabolic engineering of Saccharomyces cerevisiae for production of fatty acid-derived biofuels and chemicals." Metab Eng 21: 103-113.

Saavedra, C., K. S. Tung, D. C. Amberg and e. al (1996). "Regulation of mRNA export in response to 
stress in Saccharomyces cerevisiae." Genes Dev 10: 1608-1620.

Sainsbury, S., C. Bernecky and P. Cramer (2015). "Structural basis of transcription initiation by RNA polymerase II." Nat Rev Mol Cell Biol 16(3): 129-143.

Sampath, V. and P. Sadhale (2005). "Rpb4 and Rpb7: a sub-complex integral to multi-subunit RNA polymerases performs a multitude of functions." IUBMB Life 57(2): 93-102.

Samuel, D., T. K. S. Kumar, G. Ganesh, G. Jayaraman, P.-W. Yang, M.-M. Chang, V. D. Trivedi, S.-L. Wang, K.-C. Hwang, D.-K. Chang and C. Yu (2000). "Proline inhibits aggregation during protein refolding." Protein Science 9: 344-352.

Santos, C. N. and G. Stephanopoulos (2008). "Combinatorial engineering of microbes for optimizing cellular phenotype." Curr Opin Chem Biol 12(2): 168-176.

Santos, C. N. and G. Stephanopoulos (2008). "Combinatorial engineering of microbes for optimizing cellular phenotype." Curr Opin Chem Biol 12(2): 168-176.

Santos, C. N. and G. Stephanopoulos (2008). "Melanin-based high-throughput screen for L-tyrosine production in Escherichia coli." Appl Environ Microbiol 74(4): 1190-1197.

Schrader, M. and H. D. Fahimi (2004). "Mammalian peroxisomes and reactive oxygen species." Histochem Cell Biol 122(4): 383-393.

Shao, Z., H. Zhao and H. Zhao (2009). "DNA assembler, an in vivo genetic method for rapid construction of biochemical pathways." Nucleic Acids Res 37(2): e16.

Shi, D. J., C. L. Wang and K. M. Wang (2009). "Genome shuffling to improve thermotolerance, ethanol tolerance and ethanol productivity of Saccharomyces cerevisiae." J Ind Microbiol Biotechnol 36(1): 139147.

Shi, S., Y. Chen, V. Siewers and J. Nielsen (2014). "Improving production of malonyl coenzyme A- 
derived metabolites by abolishing Snf1-dependent regulation of Acc1." MBio 5(3): e01130-01114.

Shi, S., J. O. Valle-Rodríguez, S. Khoomrung, V. Siewers and J. Nielsen (2012). "Functional expression and characterization of five wax ester synthases in Saccharomyces cerevisiae and their utility for biodiesel production." Biotechnol Biofuels 5(7).

Shi, X., H. Wang, H. Wang, Z. Wang and C. Meng (2012). "Transferring chromosome DNA fragments from multiple donor cells into a host strain for yeast strain improvement." Can J Microbiol 58(6): 760766.

Shiba, Y., E. M. Paradise, J. Kirby, D. K. Ro and J. D. Keasing (2007). "Engineering of the pyruvate dehydrogenase bypass in Saccharomyces cerevisiae for high-level production of isoprenoids." Metabolic Engineering 9(2): 160-168.

Shiba, Y., E. M. Paradise, J. Kirby, D. K. Ro and J. D. Keasling (2007). "Engineering of the pyruvate dehydrogenase bypass in Saccharomyces cerevisiae for high-level production of isoprenoids." Metab Eng 9(2): 160-168.

Shin, S.-Y., S.-M. Jung, M.-D. Kim, N. S. Han and J.-H. Seoa (2012). "Production of resveratrol from tyrosine in metabolically engineered Saccharomyces cerevisiae." Enzyme Microb Technol 51(4): 211216.

Shivaswamy, S. and V. R. Iyer (2008). "Stress-dependent dynamics of global chromatin remodeling in yeast: dual role for SWI/SNF in the heat shock stress response." Mol Cell Biol 28(7): 2221-2234.

Si, T., Y. Luo, H. Xiao and H. Zhao (2014). "Utilizing an endogenous pathway for 1-butanol production in Saccharomyces cerevisiae." Metab Eng 22: 60-68.

Sims, R. E., W. Mabee, J. N. Saddler and M. Taylor (2010). "An overview of second generation biofuel technologies." Bioresour Technol 101(6): 1570-1580. 
Singer, M. A. and S. Lindquist (1998). "Multiple effects of trehalose on protein folding in vitro and in vivo." Mol Cell 1(4): 639-648.

Smirnoff, N. and Q. J. Cumbes (1989). "Hydroxyl radical scavenging activity of compatible solutes." Phytochemistry 28(4): 1057-1089.

Sonderegger, M. and U. Sauer (2003). "Evolutionary Engineering of Saccharomyces cerevisiae for Anaerobic Growth on Xylose." Applied and Environmental Microbiology 69(4): 1990-1998.

Spencer, J., T. G. Phister, K. A. Smart and D. Greetham (2014). "Tolerance of pentose utilising yeast to hydrogen peroxide-induced oxidative stress." BMC Res Notes 17(7): 151.

Stanley, D., A. Bandara, S. Fraser, P. J. Chambers and G. A. Stanley (2010). "The ethanol stress response and ethanol tolerance of Saccharomyces cerevisiae." J Appl Microbiol 109(1): 13-24.

Stanley, D., P. J. Chambers, G. A. Stanley, A. Borneman and S. Fraser (2010). "Transcriptional changes associated with ethanol tolerance in Saccharomyces cerevisiae." Appl Microbiol Biotechnol 88(1): 231239.

Stanley, D., S. Fraser, P. J. Chambers, P. Rogers and G. A. Stanley (2010). "Generation and characterisation of stable ethanol-tolerant mutants of Saccharomyces cerevisiae." J Ind Microbiol Biotechnol 37(2): 139-149.

Steen, E. J., R. Chan, N. Prasad, S. Myers, C. J. Petzold, A. Redding, M. Ouellet and J. D. Keasling (2008). "Metabolic engineering of Saccharomyces cerevisiae for the production of n-butanol." Microb Cell Fact 7: 36 .

Strijbis, K. and B. Distel (2010). "Intracellular acetyl unit transport in fungal carbon metabolism." Eukaryot Cell 9(12): 1809-1815.

Swinnen, S., K. Schaerlaekens, T. Pais, J. Claesen, G. Hubmann, Y. Yang, M. Demeke, M. R. Foulquie- 
Moreno, A. Goovaerts, K. Souvereyns, L. Clement, F. Dumortier and J. M. Thevelein (2012).

"Identification of novel causative genes determining the complex trait of high ethanol tolerance in yeast using pooled-segregant whole-genome sequence analysis." Genome Res 22(5): 975-984.

Szabados, L. and A. Savoure (2010). "Proline: a multifunctional amino acid." Trends Plant Sci 15(2): 89-

97.

Takagi, H. (2008). "Proline as a stress protectant in yeast: physiological functions, metabolic regulations, and biotechnological applications." Appl Microbiol Biotechnol 81(2): 211-223.

Takagi, H., M. Takaoka, A. Kawaguchi and Y. Kubo (2005). "Effect of L-proline on sake brewing and ethanol stress in Saccharomyces cerevisiae." Appl Environ Microbiol 71(12): 8656-8662.

Takahashi, T., H. Shimoi and K. Ito (2001). "Identification of genes required for growth under ethanol stress using transposon mutagenesis in Saccharomyces cerevisiae." Mol Genet Genomics 265( ): $1112-$ 1119.

Takemura, R., Y. Inoue and S. Izawa (2004). "Stress response in yeast mRNA export factor: reversible changes in Rat8p localization are caused by ethanol stress but not heat shock." J Cell Sci 117(18): 41894197.

Tan, F. R., L. C. Dai, B. Wu, H. Qin, Z. X. Shui, J. L. Wang, Q. L. Zhu, Q. C. Hu, Z. Y. Ruan and M. X. He (2015). "Improving furfural tolerance of Zymomonas mobilis by rewiring a sigma factor RpoD protein." Appl Microbiol Biotechnol 99(12): 5363-5371.

Tang, X. and W. N. Chen (2014). "Investigation of fatty acid accumulation in the engineered Saccharomyces cerevisiae under nitrogen limited culture condition." Bioresour Technol 162: 200-216. Tang, X., H. Feng and W. N. Chen (2013). "Metabolic engineering for enhanced fatty acids synthesis in Saccharomyces cerevisiae." Metab Eng 16: 95-102. 
Tehlivets, O., K. Scheuringer and S. D. Kohlwein (2007). "Fatty acid synthesis and elongation in yeast." Biochim Biophys Acta 1771(3): 255-270.

Teixeira, M. C., L. R. Raposo, N. P. Mira, A. B. Lourenco and I. Sa-Correia (2009). "Genome-wide identification of Saccharomyces cerevisiae genes required for maximal tolerance to ethanol." Appl Environ Microbiol 75(18): 5761-5772.

Thammasittirong, S. N.-R., T. Thirasaktana, A. Thammasittirong and M. Srisodsuk (2013). "Improvement of ethanol production by ethanol-tolerant Saccharomyces cerevisiae UVNR56." SpringerPlus 2: 583.

Thatipamala, R., S. Rohani and G. A. Hill (1992). "Effects of high product and substrate inhibitions on the kinetics and biomass and product yields during ethanol batch fermentation." Biotechnol Bioeng 40(2): 289-297.

Trabalzini, L., A. Paffetti, A. Scaloni, F. Talamo, E. Ferro, G. Coratza, L. Bovalini, P. Lusini, P. Martelli and A. Santucci (2003). "Proteomic response to physiological fermentation stresses in a wild-type wine strain of Saccharomyces cerevisiae." Biochem. J 370: 35-46.

Ubersax, J. A. and D. M. Platt (2010). Genetically modified microbes producing isoprenoids. . U. S. Patent. USA.

Vadali, R. V., G. N. Bennett and K. Y. San (2004). "Cofactor engineering of intracellular CoA/acetylCoA and its effect on metabolic flux redistribution in Escherichia coli." Metab Eng 6(2): 133-139.

van Roermund, C. W., H. R. Waterham, L. Ijlst and R. J. Wanders (2003). "Fatty acid metabolism in Saccharomyces cerevisiae." Cell Mol Life Sci 60(9): 1838-1851.

Vanegas, J. M., M. F. Contreras, R. Faller and M. L. Longo (2012). "Role of unsaturated lipid and ergosterol in ethanol tolerance of model yeast biomembranes." Biophys J 102(3): 507-516. 
Vemuri, G. N., M. A. Eiteman, J. E. McEwen, L. Olsson and J. Nielsen (2007). "Increasing NADH oxidation reduces overflow metabolism in Saccharomyces cerevisiae." Proc Natl Acad Sci U S A 104(7): $2402-2407$.

Verbruggen, N., X.-J. Hua, M. May and M. V. Montagu (1996). "Environmental and developmental signals modulate proline homeostasis evidence for a negative transcriptional regulator." Proc. Natl. Acad. Sci 93(8): 8787-8791.

Voorst, F. v., J. Houghton-Larsen, L. Jønson, M. C., Kielland-Brandt and A. Brandt (2006). "Genomewide identification of genes required for growth of Saccharomyces cerevisiae under ethanol stress." Yeast 23(5): 351-359.

Vriesekoop, F., C. Haass and N. B. Pamment (2009). "The role of acetaldehyde and glycerol in the adaptation to ethanol stress of Saccharomyces cerevisiae and other yeasts." FEMS Yeast Res 9(3): 365371.

Wang, B. L., A. Ghaderi, H. Zhou, J. Agresti, D. A. Weitz, G. R. Fink and G. Stephanopoulos (2014). "Microfluidic high-throughput culturing of single cells for selection based on extracellular metabolite production or consumption." Nat Biotechnol 32(5): 473-478.

Wang, G., T. Hosaka and K. Ochi (2008). "Dramatic activation of antibiotic production in Streptomyces coelicolor by cumulative drug resistance mutations." Appl Environ Microbiol 74(9): 2834-2840.

Wang, H., B. Ji, H. Ren and C. Meng (2014). "The relationship between lysine 4 on histone H3 methylation levels of alcohol tolerance genes and changes of ethanol tolerance in Saccharomyces cerevisiae." Microb Biotechnol 7(4): 307-314.

Wang, J., Y. Zhang, Y. Chen, M. Lin and Z. Lin (2012). "Global regulator engineering significantly improved Escherichia coli tolerances toward inhibitors of lignocellulosic hydrolysates." Biotechnol 
Bioeng 109(12): 3133-3142.

Wang, Y., H. Chen and O. Yu (2014). "A plant malonyl-CoA synthetase enhances lipid content and polyketide yield in yeast cells." Appl Microbiol Biotechnol 98(12): 5435-5447.

Wang, Y., Y. Li, X. Pei, L. Yu and Y. Feng (2007). "Genome-shuffling improved acid tolerance and Llactic acid volumetric productivity in Lactobacillus rhamnosus." J Biotechnol 129(3): 510-515.

Watanabe, M., K. Tamura, J. P. Magbanua, K. Takano, K. Kitamoto, H. Kitagaki, T. Akao and H. Shimoi (2007). "Elevated expression of genes under the control of stress response element (STRE) and Msn2p in an ethanol-tolerance sake yeast Kyokai no. 11." J Biosci Bioeng 104(3): 163-170.

Watanabe, M., D. Watanabe, T. Akao and H. Shimoi (2009). "Overexpression of MSN2 in a sake yeast strain promotes ethanol tolerance and increases ethanol production in sake brewing." J Biosci Bioeng 107(5): 516-518.

Watson, T. G. (1970). "Effects of Sodium Chloride on Steady-state Growth and Metabolism of Saccharomyces cerevisiae." J Gen Microbiol 64(1): 91-99.

Wattanachaisaereekul, S., A. E. Lantz, M. L. Nielsen and J. Nielsen (2008). "Production of the polyketide 6-MSA in yeast engineered for increased malonyl-CoA supply." Metab Eng 10(5): 246-254.

Wenshan, L., Z. Bo and J. Rongrong (2016). "Engineering the acetyl-CoA supply biosynthesis in Saccharomyces cerevisiae by pantothenate kinase and PDH bypass overexpression." Biotech Biofuels

\section{Submitted.}

Wickner, R. B., E. Bezsonov and D. A. Bateman (2014). "Normal levels of the antiprion proteins Btn2 and Cur1 cure most newly formed [URE3] prion variants." Proc Natl Acad Sci U S A 111(26): E27112720.

Wong, C. M., Y. Zhou, R. W. Ng, H. F. Kung Hf and D. Y. Jin (2002). "Cooperation of yeast 
peroxiredoxins Tsa1p and Tsa2p in the cellular defense against oxidative and nitrosative stress." J Biol Chem 277(7): 5385-5394.

Woychik, N. A. and M. Hampsey (2002). "The RNA Polymerase II Machinery Structure Illuminates Function." Cell 108(4): 453-463.

Yang, K.-M., J.-M. Woo, S.-M. Lee and J.-B. Park (2013). "Improving ethanol tolerance of Saccharomyces cerevisiae by overexpressing an ATP-binding cassette efflux pump." Chemical Engineering Science 103: 74-78.

Yazawa, H., H. Iwahashi and H. Uemura (2007). "Disruption of URA7 and GAL6 improves the ethanol tolerance and fermentation capacity of Saccharomyces cerevisiae." Yeast 24(7): 551-560.

Yazawa, H., Y. Kamisaka, K. Kimura, M. Yamaoka and H. Uemura (2011). "Efficient accumulation of oleic acid in Saccharomyces cerevisiae caused by expression of rat elongase 2 gene (rELO2) and its contribution to tolerance to alcohols." Appl Microbiol Biotechnol 91(6): 1593-1600.

Yenush, L., S. Merchan, J. Holmes and R. Serrano (2005). "pH-Responsive, posttranslational regulation of the Trk1 potassium transporter by the type 1-related Ppzl phosphatase." Mol Cell Biol 25(19): 86838692.

Yoshikawa, K., T. Tanaka, C. Furusawa, K. Nagahisa, T. Hirasawa and H. Shimizu (2009). "Comprehensive phenotypic analysis for identification of genes affecting growth under ethanol stress in Saccharomyces cerevisiae." FEMS Yeast Res 9(1): 32-44.

You, K. M., C. L. Rosenfield and D. C. Knipple (2003). "Ethanol Tolerance in the Yeast Saccharomyces cerevisiae Is Dependent on Cellular Oleic Acid Content." Applied and Environmental Microbiology 69(3): 1499-1503.

Zara, G., L. Bardi, S. Belviso, G. A. Farris, S. Zara and M. Budroni (2008). "Correlation between cell 
lipid content, gene expression and fermentative behaviour of two Saccharomyces cerevisiae wine strains." J Appl Microbiol 104(3): 906-914.

Zhang, F., S. Rodriguez and J. D. Keasling (2011). "Metabolic engineering of microbial pathways for advanced biofuels production." Curr Opin Biotechnol 22(6): 775-783.

Zhang, H., H. Chong, C. B. Ching and R. Jiang (2012). "Random mutagenesis of global transcription factor cAMP receptor protein for improved osmotolerance." Biotechnol Bioeng 109(5): 1165-1172.

Zhang, H., H. Chong, C. B. Ching, H. Song and R. Jiang (2012). "Engineering global transcription factor cyclic AMP receptor protein of Escherichia coli for improved 1-butanol tolerance." Appl Microbiol Biotechnol 94(4): 1107-1117.

Zhang, M., Y. Xiao, R. Zhu, Q. Zhang and S. L. Wang (2012). "Enhanced thermotolerance and ethanol tolerance in Saccharomyces cerevisiae mutated by high-energy pulse electron beam and protoplast fusion." Bioprocess Biosyst Eng 35(9): 1455-1465.

Zhao, H., J. Li, B. Han, X. Li and J. Chen (2014). "Improvement of oxidative stress tolerance in Saccharomyces cerevisiae through global transcription machinery engineering." J Ind Microbiol Biotechnol 41(5): 869-878.

Zhao, H., J. Li, B. Han, X. Li and J. Chen (2014). "Improvement of oxidative stress tolerance in Saccharomyces cerevisiae through global transcription machinery engineering." J Ind Microbiol Biotechnol 41(5): 869-878.

Zhao, X. Q. and F. W. Bai (2009). "Mechanisms of yeast stress tolerance and its manipulation for efficient fuel ethanol production." J Biotechnol 144(1): 23-30.

Zheng, D. Q., J. Chen, K. Zhang, K. H. Gao, O. Li, P. M. Wang, X. Y. Zhang, F. G. Du, P. Y. Sun, A. M. Qu, S. Wu and X. C. Wu (2014). "Genomic structural variations contribute to trait improvement during 
whole-genome shuffling of yeast." Appl Microbiol Biotechnol 98(7): 3059-3070. 